\title{
Luminescence and tunable color properties of uniphase white-emitting Sr3B2Si08:Tm3+/Dy3+/Eu3+ phosphors by energy transfer for UV-excited white LEDs
}

\section{Bo Yuan}

Jilin Institute of Chemical Technology

Chaochao Qi

Jilin Institute of Chemical Technology

Xiangting Zhang

Jilin University

Yanhua Song

Jilin University

\section{Guoyan Luan}

Jilin Institute of Chemical Technology

haifeng Zou ( $\square$ haifengzou0431@sohu.com )

Jilin University

\section{Research Article}

Keywords: Luminescence, tunable color properties, white LEDs, Sr 3 B 2 SiO 8 :Tm 3+ /Dy 3+ /Eu 3+, UV excited

Posted Date: April 23rd, 2021

DOl: https://doi.org/10.21203/rs.3.rs-235705/v1

License: (c) (1) This work is licensed under a Creative Commons Attribution 4.0 International License. Read Full License

Version of Record: A version of this preprint was published at Optical and Quantum Electronics on August 14th, 2021. See the published version at https://doi.org/10.1007/s11082-021-03110-8. 
Luminescence and tunable color properties of uniphase white-emitting

$\mathrm{Sr}_{3} \mathrm{~B}_{2} \mathrm{SiO}_{8}: \mathrm{Tm}^{3+} / \mathrm{Dy}^{3+} / \mathrm{Eu}^{3+}$ phosphors by energy transfer for UV-excited white LEDs

\author{
Bo Yuan ${ }^{1}$, Chaochao $\mathrm{Qi}^{1}$, Xiangting Zhang ${ }^{2}$, Yanhua Song ${ }^{2}$, Guoyan Luan ${ }^{1 *}$, Haifeng Zou ${ }^{2 *}$ \\ ${ }^{1}$ Institute of Petrochemical Technology, Jilin Institute of Chemical Technology, Jilin 132022, PR \\ China \\ ${ }^{2}$ College of Chemistry, Jilin University, Changchun 130012, PR China
}

*Corresponding author. Tel: +86 43185155275 .

E-mail address: zouhf@jlu.edu.cn

Key words $\mathrm{Sr}_{3} \mathrm{~B}_{2} \mathrm{SiO}_{8}: \mathrm{Tm}^{3+} / \mathrm{Dy}^{3+} / \mathrm{Eu}^{3+}$. Energy transfer $\cdot$ Multi-color emission $\cdot$ WLED

\begin{abstract}
Multi-color single-phase white emission $\mathrm{Sr}_{3} \mathrm{~B}_{2} \mathrm{SiO}_{8}: \mathrm{Tm}^{3+} / \mathrm{Dy}^{3+} / \mathrm{Eu}^{3+}$ fluorescent powders were prepared by solid-state fritting method. The fluorescence performance of $\mathrm{Tm}^{3+}$, $\mathrm{Dy}^{3+}$ and $\mathrm{Eu}^{3+}$ ions single-activated $\mathrm{Sr}_{3} \mathrm{~B}_{2} \mathrm{SiO}_{8}$ phosphors were investigated and they exhibit desirable behaviors in their characteristic emissions. Meanwhile, the energy required for migration from $\mathrm{Tm}^{3+}$ to $\mathrm{Dy}^{3+}$ and from $\mathrm{Dy}^{3+}$ to $\mathrm{Eu}^{3+}$ in co-doped fluorescent substance was determined by the aids of steady-state and transient-state photoluminescence spectra/decay measurements. The energy transmission process from $\mathrm{Tm}^{3+}$ to $\mathrm{Dy}^{3+}$ and $\mathrm{Dy}^{3+}$ to $\mathrm{Eu}^{3+}$ has been explored to be a dipole-quadrupole interaction and a dipole-dipole interaction of the resonant type, respectively. More significantly, by tuning the molarity ratio of doping $\mathrm{Dy}^{3+} / \mathrm{Eu}^{3+}$ ions, multicolor emission from yellow to red shall be achieved in $\mathrm{Sr}_{3} \mathrm{~B}_{2} \mathrm{SiO}_{8}: \mathrm{Dy}^{3+}, \mathrm{Eu}^{3+}$ phosphors for its potential adhibitions in solid-state lighting applications. The $\mathrm{Sr}_{3} \mathrm{~B}_{2} \mathrm{SiO}_{8}: \mathrm{Tm}^{3+}$, Dy ${ }^{3+}$ samples can implement white light emission by the appropriate changeable doping molar quantity of $\mathrm{Tm}^{3+}$ and $\mathrm{Dy}^{3+}$, applying potentially in white LEDs. The energy migrations between co-doped rare earth ions play a decisive role in these processes.
\end{abstract}




\section{Introduction}

Considered as the significant emerging lighting source, white light emitting diodes (WLEDs) are infiltrating the global optics space with a high speed of development due to their unique features of high luminous yield, long service life and small space usage (Chen et al. 2014; Bharat 2016; Shang et al. 2014; Liu et al. 2018). Nowadays, the core technology of commercializing white LED is packaging of broadband yellow phosphor YAG:Ce ${ }^{3+}$ with InGan blue chip to switch blue photons to long-wavelength ones (Wang et al. 2016). Unfortunately, the unsolved challenging issues of yellowing and red component absence leading to the decrease of white light softness and color rendering for the combination restrict its expansion in relevant application (Ye et al. 2010; Smet 2011). A new format of realizing white LED with ultraviolet chip coating red, green, and blue multiphase phosphors was introduced to ameliorate the deficiency of blue chip system. It is a pity that the serious energy reabsorption and proportion imbalance occurs in the mixing of multi-component phosphors, which have a certain impact on the luminous efficiency and color reductivity (Liu et al. 2014; Luwang 2011; Zhu et al. 2007). Under this circumstance, another effective alternate was major concentrated in the single-phase phosphor with white or multi-color emission, which was more stimulated in the ultraviolet region. In generally, white or multi-color emission could be carried out by controlling the luminous color of materials effectively, on account of designing the energy transfer between different sensitizer/activator ions in the single-phase substrate (Patnam 2019; Zhu et al. 2014; Chen et al. 2012). Therefore, seeking for novel single-phase phosphors and high efficient energy transfer has become greatly in demand.

Rare earth ions as the frequently used phosphor activators and sensitizers have been playing a critical part in pc-WLEDs due to the ample emission colors range from near-infrared to ultraviolet (Song et al. 2015; Setlur 2009). It is common knowledge that $\mathrm{Tm}^{3+}$ ions possessing of $4 \mathrm{f}^{12}$ electron configuration are generally served as blue-light emission active centers, which is mainly attributed to the characteristic level transition ${ }^{1} \mathrm{D}_{2} \rightarrow{ }^{3} \mathrm{~F}_{4}$ (Hu et al. 2014). Comfortable white light is more likely to be emerged in the co-doped system with $\mathrm{Tm}^{3+}$ ions as sensitizers. Furthermore, owing to ${ }^{4} \mathrm{~F}_{9 / 2} \rightarrow{ }^{6} \mathrm{H}_{13 / 2}$ and ${ }^{4} \mathrm{~F}_{9 / 2} \rightarrow{ }^{6} \mathrm{H}_{15 / 2}$ transitions of Dy ${ }^{3+}$ ions, the emission color is centralized in the blue and yellow range of the visible spectrum, respectively (Pavitra 2013). Hence, there is adequate possibility for $\mathrm{Tm}^{3+}$ to behave as a sensitizing donor which diverts energy to $\mathrm{Dy}^{3+}$ in co-doped system for white light being engendered by the recombination of different light color. 
Deemed as excellent red emitter for phosphor, the $\mathrm{Eu}^{3+}$ ion exhibits efficient typical emissions belonging to the transition from upper states ${ }^{5} \mathrm{D}_{0,1,2}$ to ground states ${ }^{7} \mathrm{~F}_{1,2,3,4,5}$ (Huang et al. 2017). Thus, by adding $\mathrm{Eu}^{3+}$ ions into the same host lattice for offering intense red composition, the deficiency of red component can be conquered in the Dy ${ }^{3+}$ single-doped phosphor. Remarkably, the $\mathrm{Tm}^{3+} \rightarrow \mathrm{Dy}^{3+}$ and $\mathrm{Dy}^{3+} \rightarrow \mathrm{Eu}^{3+}$ energy transfer is studied in very few material families as white or multi-color emitting phosphor for construction of w-LEDs, such as $\mathrm{K}_{3} \mathrm{Gd}\left(\mathrm{PO}_{4}\right)_{2}: \mathrm{Tm}^{3+}, \mathrm{Dy}^{3+}$ (Zhao et al. 2017), NaGdTiO4: $\mathrm{Tm}^{3+}, \mathrm{Dy}^{3+}\left(\right.$ Bharat 2016), $\mathrm{NaBi}\left(\mathrm{MoO}_{4}\right)_{2}: \mathrm{Dy}^{3+}, \mathrm{Eu}^{3+}$ (Li et al. 2019) and $\mathrm{Ca}_{2} \mathrm{ZnSi}_{2} \mathrm{O}_{7}: \mathrm{Dy}^{3+}, \mathrm{Eu}^{3+}$ (Mondal 2018). For all that, the strict synthesis conditions and not high luminescence efficiency of these materials hamper their further applications.

The luminescent material host of borosilicate for rare earth ions emission has attracted the interest of numerous researchers because of their economical prepared temperature and prominent corrosion resistance (Chang et al. 2007). Among the borosilicate materials, $\mathrm{Sr}_{3} \mathrm{~B}_{2} \mathrm{SiO}_{8}$ has been affirmed as an excellent matrix with good application prospects in the phosphor-converted white LEDs, for example, $\mathrm{Sr}_{3} \mathrm{~B}_{2} \mathrm{SiO}_{8}: \mathrm{Eu}^{3+}$ (Wang et al. 2011), $\mathrm{Sr}_{3} \mathrm{~B}_{2} \mathrm{SiO}_{8}: \mathrm{Eu}^{2+}$, $\mathrm{Dy}^{3+}$ (Sabikoglu 2013), $\mathrm{Sr}_{3} \mathrm{~B}_{2} \mathrm{SiO}_{8}: \mathrm{Sm}^{3+}$ (Sun et al. 2016). However, to our knowledge, white light emitting and tunable color properties on the strength of energy transfer between active centers, $\mathrm{Tm}^{3+} \rightarrow \mathrm{Dy}^{3+}$, $\mathrm{Dy}^{3+} \rightarrow \mathrm{Eu}^{3+}$ has not been yet reported in $\mathrm{Sr}_{3} \mathrm{~B}_{2} \mathrm{SiO}_{8}$ system. Herein, we present the design of novel $\mathrm{Sr}_{3} \mathrm{~B}_{2} \mathrm{SiO}_{8}: \mathrm{Tm}^{3+} / \mathrm{Dy}^{3+} / \mathrm{Eu}^{3+}$ phosphor synthesized by solid-state fritting reaction which shows unique white light emitting and tunable characteristics. Additionally, the energy transfer of $\mathrm{Tm}^{3+} \rightarrow \mathrm{Dy}^{3+}$ and $\mathrm{Dy}^{3+} \rightarrow \mathrm{Eu}^{3+}$ has been confirmed to exist in the $\mathrm{Sr}_{3} \mathrm{~B}_{2} \mathrm{SiO}_{8}$ matrix and the mechanism were discussed indetail on account of steady-state and transient-state photoluminescence spectra/decay consequences. All results indicated that the tunable color from yellow to red luminescence has been obtained in $\mathrm{Sr}_{3} \mathrm{~B}_{2} \mathrm{SiO}_{8}: \mathrm{Dy}^{3+}, \mathrm{Eu}^{3+}$ phosphor by the alteration of $\mathrm{Dy}^{3+} / \mathrm{Eu}^{3+}$ molarity ratio and the phosphor $\mathrm{Sr}_{3} \mathrm{~B}_{2} \mathrm{SiO}_{8}: \mathrm{Tm}^{3+}, \mathrm{Dy}^{3+}$ is a potential candidate for warm UV or near-UV WLEDs.

\section{Experimental}

\subsection{Synthesis procedure}

Phosphors with the chemical constituents of $\mathrm{Sr}_{3} \mathrm{~B}_{2} \mathrm{SiO}_{8}: \mathrm{Tm}^{3+} / \mathrm{Dy}^{3+} / \mathrm{Eu}^{3+}$ were prepared via the traditional solid-state process. The starting materials such as $\mathrm{SrCO}_{3}, \mathrm{H}_{3} \mathrm{BO}_{3}, \mathrm{SiO}_{2}, \mathrm{Li}_{2} \mathrm{CO}_{3}$ and $\mathrm{Tm}_{2} \mathrm{O}_{3}(99.99 \%), \mathrm{Dy}_{2} \mathrm{O}_{3}(99.99 \%), \mathrm{Eu}_{2} \mathrm{O}_{3}(99.99 \%)$ were blended in accordance with the 
chemometry proportion, and well-milled evenly for $30 \mathrm{~min}$ in an agate mortar and pestle. All the starting chemicals were of the analytical reagent grade. The precursors were diverted into corundum crucibles, then calcined at $600{ }^{\circ} \mathrm{C}$ for $1 \mathrm{~h}$ in elema stove. Since removed, secondary milled and programmed temperature up to $1080{ }^{\circ} \mathrm{C}$, holding for $3 \mathrm{~h}$ in a tube furnace under ambient atmosphere. After the reaction, nature air cooling and milled again into smalls to acquire the final products.

\subsection{Characterization}

The poly-crystalline phases of $\mathrm{Sr}_{3} \mathrm{~B}_{2} \mathrm{SiO}_{8}: \mathrm{Tm}^{3+} / \mathrm{Dy}^{3+} / \mathrm{Eu}^{3+}$ phosphors were analyzed by $\mathrm{X}$-ray powder diffraction (XRD) on a Rigaku Miniflex 600, with $\mathrm{Cu} \mathrm{K} \alpha$ radiation. The diffraction patterns were scanned at $6^{\circ} \mathrm{min}^{-1}$ from $10^{\circ}$ to $60^{\circ}$. The refinements of crystal structure were operated applying GSAS program based on the Rietveld method. The steady-state photoluminescence excitation/emission spectra and the quantum efficiency (QE) were measured (Edinburgh FS5) using a 150W Xenon lamp as the excitation source and an integrating sphere. The transient-state luminescence decay measurements were obtained (Edinburgh FLS920 spectrophotometer) utilizing $350 \mathrm{~W}$ Xenon lamps as the excitation source. The element composition and content of phosphors was inspected by using a scanning electron microscope (SEM, JSM-6490LV) equipped with an energy-dispersive X-ray spectrometer (EDS). The electronic structure of SBSO was evaluated on a personal computer by using the module CASTAP in the software Materials Studio based on the density functional theory (DFT). The particle diameter distribution of phosphors was carried out using particle size and Zeta potential analyzer (NanoBrook Omni).

\section{Results and discussion}

\subsection{Crystalline characteristic and Phase purity analysis}

The as recorded, computed and difference XRD patterns obtained by Rietveld refinement corresponding to SBSO are represented in Figure 1. The weighted and profile R-factors, Rwp, Rp and $\chi^{2}$ computed after multiple iterations are intended to be $11.29 \%, 7.48 \%$ and 1.09 , respectively, well fitted the reflection condition. The raw structure model (Figure 2) were constructed consulting to previously literature for SBSO (JCPDS 32-1224). The synthesized sample SBSO is pure and has the orthorhombic symmetry structure (space group: Pnma) and the lattice constants are computed as $a=12.361 \AA, b=3.927 \AA, c=5.419 \AA$. The unit cell parameters are recorded as 
$\mathrm{V}=263.05 \AA^{3}$ and $\mathrm{Z}=4$. The refinement crystallographic parameters of lattice structure, the coordinates of the atom as well as the occupancy ratio are presented in Tables1 and 2. Figure 3 illustrates the representative XRD patterns for $\mathrm{SBSO}: \mathrm{Tm}^{3+} / \mathrm{Dy}^{3+} / \mathrm{Eu}^{3+}$ phosphors with diverse doping concentration and also the powder diffraction standard of SBSO (JCPDS 32-1224). The measured XRD patterns revealed the crystal structure match of different doping samples with standard diffraction pattern corresponding to JCPDS 32-1224. The absence of secondary phases confirm that the doping level of $\mathrm{Tm}^{3+}, \mathrm{Dy}^{3+}$ and $\mathrm{Eu}^{3+}$ ions is within the solubility limit and the small introduction is stabilized in the crystal structure of the matrix without inducing significant effect. The ionic radii values for 8 coordination number indicate that the $\mathrm{Tm}^{3+}\left(1.134 \AA\right.$ ), $\mathrm{Dy}^{3+}$ $(0.908 \AA)$ and $\mathrm{Eu}^{3+}(0.950 \AA)$ ions should occupy the lattice position of $\mathrm{Sr}^{3+}(1.18 \AA)$ rather than $\mathrm{B}^{3+}$ after doping into the crystal lattice (Bharat 2016).

The energy-dispersive spectrometer (EDS) of SBSO:7\% $\mathrm{Dy}^{3+}$ samples were studied to further analyze the element composition and content. As shown in Figure 4, the EDX spectrum results confirm the presence of boron (B), oxygen $(\mathrm{O})$, silicon $(\mathrm{Si})$, strontium $(\mathrm{Sr})$ and dysprosium (Dy) elements in $\mathrm{Sr}_{3} \mathrm{~B}_{2} \mathrm{SiO}_{8}: 0.07 \mathrm{Dy}^{3+}$ sample, no other peaks of impurity elements were detected. In addition, from the results of quantitative analysis (Table 3), it can be seen that the values of Weight $\%$ and Atomic\% lists are in agreement with the stoichiometric ratio of chemical formula $\mathrm{Sr}_{3} \mathrm{~B}_{2} \mathrm{SiO}_{8}: 0.07 \mathrm{Dy}^{3+}$ basically. Therefore, the information of EDS gives further support for the XRD analysis.

\subsection{Luminescence and energy transfer characteristics of SBSO:Tm ${ }^{3+}, \mathrm{Dy}^{3+}$}

The normalized photoluminescence excitation and emission spectra of SBSO:3\% $\mathrm{Tm}^{3+}$ sample is displayed in Figure 5. It can be seen that a sharp absorption peak at $357 \mathrm{~nm}$ corroborated with the f-f parity-forbidden $\mathrm{Tm}^{3+} \cdot{ }^{3} \mathrm{H}_{6} \rightarrow{ }^{1} \mathrm{D}_{2}$ appears in the excitation spectrum when monitored at $456 \mathrm{~nm}$, which confirms a perfect match with the UV-LED chip bands. Upon the stimulation of $357 \mathrm{~nm}$ irradiation, an intense blue emission locating at about $456 \mathrm{~nm}$ is observed assigned to the shift of $\mathrm{Tm}^{3+}$ from ${ }^{1} \mathrm{D}_{2}$ to ${ }^{3} \mathrm{~F}_{4}$. The special characteristic luminescence indicates that $\mathrm{Tm}^{3+}$ ions are appropriate as sensitizers to transfer energy while comfortable white light is more likely to be obtained in co-doping system.

On the side, the comparison result of the emission curves of SBSO: $\mathrm{x} \% \mathrm{Tm}^{3+}(\mathrm{x}=0.5-5)$ were presented in the upper inset Figure 5. It can be surveyed that the emission intensities of $\mathrm{Tm}^{3+}$ 
heighten sustainably with the doping content raising until the concentration is reaching $3 \mathrm{~mol} \%$ (the emission intensity is at the maximum position), then showed a conspicuous monotonous decrement for dopant level increase, ascribed to the attainment of solubility limit. The concentration quenching of $\mathrm{Tm}^{3+}$ ions is a physical phenomenon of nonradiative relaxation, that is, the luminescence center returns to the ground state from the excited state in nonradiative pattern. In this case, the energy emitted by the system will be lost in the lattice in form of vibrational kinetic energy. The theoretical formula of nonradiative transition probability is as follows:

$\mathrm{W}=\mathrm{Ce}^{-\alpha \Delta \mathrm{E}}(1+\mathrm{m})^{\Delta \mathrm{E} / \mathrm{hu}}$

where $\triangle \mathrm{E}$ is energy difference between the excited state and the ground state, ho represents photon energy ( $v$ is the ambient vibration frequency), $\mathrm{C}$ and $\alpha$ are proportional constants depending on matrix, and $\mathrm{m}$ is a temperature dependent parameter (Blass 1979). It can be seen from the formula that the nonradiative transition probability (W) is only related to $\triangle \mathrm{E}$ and ho when the environment and matrix material are determined. When the concentration of luminescent centers increases, the distance between ions decreases, so that the electron-lattice coupling and interaction strength increases. Under the circumstances, energy transfer occurs among the neighboring centers (hv), and $\mathrm{W}$ will raise with the enhancing of ho according to the equation. It means, the excitation energy is transferred from the absorption site to the nonradiative depletion site, and then lost in the lattice of system in form of vibrational energy, the concentration quenching occurs. Thus, the energy transfer from one activator to another will strongly depend on the distance $(\mathrm{R})$ between $\mathrm{Tm}^{3+}$ ions, when the value reaches the critical transfer distance $\left(\mathrm{R}_{\mathrm{c}}\right)$, the concentration quenching will take place. Blasse has pointed out that the critical transfer distance $\left(\mathbf{R}_{c}\right)$ is approximately equal to twice the radius of a sphere with this volume (Blass 1969):

$\mathrm{R}_{\mathrm{c}}=2\left[\frac{3 \mathrm{~V}}{4 \pi \mathrm{X}_{\mathrm{c}} \mathrm{N}}\right]^{1 / 3}$

where $\mathrm{X}_{\mathrm{c}}$ is the critical concentration, $\mathrm{N}$ is the number of cations in the unit cell and $\mathrm{V}$ is the volume of the unit cell. By taking the experimental and analytic values of $\mathrm{X}_{\mathfrak{c}}, \mathrm{N}$ and $\mathrm{V}(0.03,4$, 263.05, respectively), the critical distance $R_{c}$ is estimated by Eq.(2) to be about $15.79 \AA$.

Furthermore, based on the previous refined structure parameters, the band gap energy and orbital density of pure SBSO are calculated by using CASTAP module of Materials Studio software. As shown in Figure 6a, b, it is clear that the computed energy gap is approximately 1.7 
$\mathrm{eV}$, implying that SBSO is an appropriate luminescent host for accommodating both the ground and excited states of luminescent ions within its wide band gap. The optical band gap $\left(\mathrm{E}_{\mathrm{g}}\right)$ of SBSO: $3 \% \mathrm{Tm}^{3+}$ can be obtained from the diffuse reflectance spectrum of the sample, estimated according to the equation:

$(\alpha h v)^{\mathrm{n}}=A\left(h v-\mathrm{E}_{\mathrm{g}}\right)$

where $\alpha$ is absorption coefficient, ho represents photon energy, A is a proportional constant and the exponent $n=1 / 2$ and 2 is the allowed indirect transition and allowed direct transitions, respectively (Zhao et al. 2018). The value of $\alpha$ can be obtained from the reflection coefficient R:

$\alpha=\frac{\left(1-R^{2}\right)}{2 R}$

The graphs plotting for $(\alpha h v)^{2}$ and $(\alpha h v)^{1 / 2}$ verses ho give the straight line with intercept indicating the optical energy band gap. As shown in Figure 7, the optical band gap energy is determined to be 3.6, $3.1 \mathrm{eV}$ for direct and indirect allowed transitions of the SBSO:3\% $\mathrm{Tm}^{3+}$ phosphor, respectively. The electronic band structure of pure SBSO and SBSO:3\% $\mathrm{Tm}^{3+}$ confirms the above luminescent properties of $\mathrm{Tm}^{3+}$ ions.

As $7 \mathrm{~mol} \% \mathrm{Dy}^{3+}$ singly doped SBSO host, the PLE and PL spectra are shown in Figure 8a. It is observed that monitored with $576 \mathrm{~nm}$ emission of $\mathrm{Dy}^{3+}$, the excitation spectrum within the section of $275-500 \mathrm{~nm}$ is composed of several narrow bands centered at 296, 324, 349, 387, 425 and $452 \mathrm{~nm}$, which corresponds to the parity-forbidden f-f transitions $\left({ }^{6} \mathrm{H}_{15 / 2} \rightarrow{ }^{4} \mathrm{H}_{13 / 2},{ }^{6} \mathrm{H}_{15 / 2} \rightarrow{ }^{6} \mathrm{P}_{3 / 2}\right.$, ${ }^{6} \mathrm{H}_{15 / 2} \rightarrow{ }^{6} \mathrm{P}_{7 / 2},{ }^{6} \mathrm{H}_{15 / 2} \rightarrow{ }^{4} \mathrm{P}_{7 / 2},{ }^{6} \mathrm{H}_{15 / 2} \rightarrow{ }^{4} \mathrm{G}_{11 / 2}$ and ${ }^{6} \mathrm{H}_{15 / 2} \rightarrow{ }^{4} \mathrm{I}_{15 / 2}$ ). The emission spectrum excited by $349 \mathrm{~nm}$ arises two dominating incisive bands at around 487 and $576 \mathrm{~nm}$, ascribed to the typical energy level for Dy ${ }^{3+}$ ions, the ${ }^{4} \mathrm{~F}_{9 / 2} \rightarrow{ }^{6} \mathrm{H}_{15 / 2}$ of magnetic dipole (blue) and the ${ }^{4} \mathrm{~F}_{9 / 2} \rightarrow{ }^{6} \mathrm{H}_{13 / 2}$ of electric dipole (yellow), respectively. The stronger yellow luminescence than blue demonstrates that the crystal field of Dy ${ }^{3+}$ centersis no inversion symmetry in SBSO host (Li et al. 2019; Mondal 2018). A series of diverse emission spectra for SBSO: $x \% \mathrm{Dy}^{3+}(\mathrm{x}=1-9)$ phosphors under $349 \mathrm{~nm}$ excitation were depicted in Figure 8b. The results of this section can be discussed with reference to Figure 5 and the maximum luminous molality is $7 \mathrm{~mol} \%$ for Dy ${ }^{3+}$ ions in SBSO host.

As described in Figure 9a, amidst the incisive emission of $\mathrm{Tm}^{3+}$ and the $\mathrm{Dy}^{3+}$ absorption transitions, a manifest spectral overlap emerges ranging from 440 to $470 \mathrm{~nm}$. Moreover, the excitation spectrum for SBSO: $\mathrm{Tm}^{3+}, \mathrm{Dy}^{3+}$ (Figure 9b) reveals some absorption bands belonging to 
the feature f-f parity-forbidden of $\mathrm{Tm}^{3+}$ and $\mathrm{Dy}^{3+}$, observed by the relevant characteristic emission (456 nm of $\mathrm{Tm}^{3+}$ and $576 \mathrm{~nm}$ of $\mathrm{Dy}^{3+}$ ). All the above discussion spredict convincingly that the process of energy transfer from $\mathrm{Tm}^{3+}$ to $\mathrm{Dy}^{3+}$ is likely to occur in SBSO host. Therefore, the synergy of yellow $\left(\mathrm{Dy}^{3+}\right)$ and blue $\left(\mathrm{Tm}^{3+}\right)$ emissions via the energy transfer could be considered to achieve white light emission in a single substance upon UV irradiation stimulation.

The speciality of multi-color white light in single-phase substrate is of consequence for promoting the application of w-LEDs. Diverting energy from donor ions to acceptor ions is critical in this process ( $\mathrm{Li}$ et al. 2014; Wang et al. 2009). A series of emission spectra for SBSO:3\% $\mathrm{Tm}^{3+}, \mathrm{y} \% \mathrm{Dy}^{3+}(\mathrm{y}=0,1,3,5,7,9)$ were illustrated in Figure 10, to verify the energy migration in co-doping single-phase system. It can be revealed that the blue proper emission for $\mathrm{Tm}^{3+}$ and the yellow sharp peak for $\mathrm{Dy}^{3+}$ ions emerge simultaneously in the PL spectrum of SBSO: $\mathrm{Tm}^{3+}$, $\mathrm{Dy}^{3+}$ under the $357 \mathrm{~nm}$ excitation. With the invariant of $\mathrm{Tm}^{3+}$ doping density ( $\left.3 \mathrm{~mol} \%\right)$ while the content for $\mathrm{Dy}^{3+}$ increasing gradually, the $\mathrm{Tm}^{3+}$ emission strength decreased uniformly, to the contrary that of $\mathrm{Dy}^{3+}$ enhanced by degrees and the maximal intensity was at $\mathrm{y}=7$. Then the intensity of $\mathrm{Dy}^{3+}$ emission shows a reduction caused by the self-quenching effect which were the interactions between $\mathrm{Dy}^{3+}$ ions. In terms of the appearance above, it could reflect the fact that the energy transfer from $\mathrm{Tm}^{3+}$ to $\mathrm{Dy}^{3+}$ occurred in SBSO: $\mathrm{Tm}^{3+}, \mathrm{Dy}^{3+}$ system, while the emission of white light shall be attributed to the luminous color composition of $\mathrm{Tm}^{3+}$ and $\mathrm{Dy}^{3+}$ on account of the energy migration. This can be verified by the CIE chromaticity coordinates.

The decay curves for $\mathrm{Tm}^{3+}$ emission with variations of $\mathrm{Dy}^{3+}$ molarity in SBSO system were measured by the stimulation of $357 \mathrm{~nm}$ and monitoring at $456 \mathrm{~nm}$, to explore and certify the energy transfer thoroughly. All the decay behaviors of luminescence as displayed in Figure 11 shall be well-explained by a double-exponential relation:

$\mathrm{I}=\mathrm{I}_{0}+\mathrm{A}_{1} \exp \left(-\mathrm{t} / \tau_{1}\right)+\mathrm{A}_{2} \exp \left(-\mathrm{t} / \tau_{2}\right)$

where $\mathrm{I}_{0}$ denotes the initial luminous intensity, I denotes the emission intensity at a certain time, $\mathrm{A}_{1}, \mathrm{~A}_{2}$ are constant coefficients, $\mathrm{t}$ is time, $\tau_{1}$ and $\tau_{2}$ express the decay lifetimes for different luminous components, respectively. Furthermore, the following expression can determine the average values of decay lifetime:

$\tau_{\text {avg }}=\frac{A_{1} \tau_{1}^{2}+A_{2} \tau_{2}^{2}}{A_{1} \tau_{1}+A_{2} \tau_{2}}$ 
The lifetime values of $\mathrm{Tm}^{3+}$ ions in $\mathrm{SBSO}: 3 \% \mathrm{Tm}^{3+}, \mathrm{y} \% \mathrm{Dy}^{3+}$ system were figured out, 6.18, 4.05, $3.14,2.53$ and $2.16 \mu$ s, respectively, when $\mathrm{y}=0,3,5,7$ and 9. The energy transfer of $\mathrm{Tm}^{3+} \rightarrow \mathrm{Dy}^{3+}$ was affirmed dramatically via the tendency of lifetime for $\mathrm{Tm}^{3+}$ ions shortening monotonously with the rise in the level of $\mathrm{Dy}^{3+}$ ions.

Normally, the energy transfer efficiency $\left(\eta_{T}\right)$ from energy donor (sensitizer) to energy acceptor (activator) is worked out in terms of formula as follows (Dexter 1954):

$\eta_{\mathrm{T}}=1-\frac{\mathrm{I}_{\mathrm{S}}}{\mathrm{I}_{\mathrm{SO}}}$

where $\mathrm{IS}_{\mathrm{S}}$ and $\mathrm{I}_{\mathrm{S} o}$ express the luminous intensities of the energy donor $\left(\mathrm{Tm}^{3+}\right)$ co-doping and no co-doping the energy acceptor $\left(\mathrm{Dy}^{3+}\right)$, respectively. The relation curve of $\eta_{\mathrm{T}}$ as the function of $\mathrm{Dy}^{3+}$ doping content is presented in Figure 12. The $\eta_{\mathrm{T}}$ value increases persistently with the rising in $\mathrm{Dy}^{3+}$ concentrations nevertheless the growth rate slackens inch by inch, ultimately the energy transfer verges to saturation caused by the identical $\mathrm{Tm}^{3+}$ concentration. The peak efficiency can be up to $80 \%$ at $\mathrm{y}=9$ which reveals that it is efficient.

Through the above discussion, it can be found that there are both spectral overlap and energy level matching between $\mathrm{Tm}^{3+}$ and $\mathrm{Dy}^{3+}$ ions, indicating that the energy migration for $\mathrm{Tm}^{3+} \rightarrow \mathrm{Dy}^{3+}$ contains two kinds of manner: radiative transfer and resonant non-radiative transfer. According to Dexter's theory, the energy transfer rate for $\mathrm{Tm}^{3+} \rightarrow \mathrm{Dy}^{3+}$ can be defined by the following formula:

$\mathrm{H}_{\mathrm{DA}}=\frac{2 \pi}{\mathrm{h}}\left|<D, \mathrm{~A}^{*}\right| \mathrm{H}_{\mathrm{DA}} \mid \mathrm{D}^{*}, \mathrm{~A}>\mathrm{I} \cdot \int \mathrm{gD}(\mathrm{E}) \cdot \mathrm{gA}(\mathrm{E}) \mathrm{dE}$

where D represents energy donor, A represents energy acceptor, $\mathrm{H}_{\mathrm{DA}}$ is the Hamiltonian operator of the interaction, the integral item denotes as spectral overlap (radiative transfer) and the matrix element expresses the interaction between the initial state $\left|D^{*}, A\right\rangle$ and the final state $\mid D, A^{*}>$ (resonant transfer interaction, related to the distance between ions) (Dexter 1954). In accordance with the above expression, it can been seen that when the concentration of two centers is very low and the distance between them is large, the energy transfer depends on the spectral overlap (radiation transfer is dominant); when the center concentration increases and the distance between centers shortens to a certain range, the nonradiative transfer of electric multipole interaction occurs; if the concentration of the centers is further increased, nonradiative transfer of 
exchange interaction may appear. The probability of energy transfer for $\mathrm{Tm}^{3+} \rightarrow \mathrm{Dy}^{3+}$ in SBSO via electronic exchange interaction is excluded, since this mechanism is only effective at a shorter $R_{c}$ of $<5 \AA$ ( it can be estimated that $\mathrm{R}_{\mathrm{Tm}-\mathrm{Dy}}$ is greater than $5 \AA$ by Eq.(2)). To analyze the occurrence of electrostatic interaction for resonant energy transfer, the following expression is given with respect to the Dexter's energy transfer theory for multipolar interaction and Reisfeld's approximation (Dexter 1954; Reisfeld 1979):

$\frac{\mathrm{I}_{\mathrm{SO}}}{\mathrm{I}_{\mathrm{S}}} \propto \mathrm{C}^{\frac{\mathrm{n}}{3}}$

where $\mathrm{C}$ is the sum of molarity for all doping ions $\left(\mathrm{Tm}^{3+}\right.$ and $\left.\mathrm{Dy}^{3+}\right)$; Eq.(9) for $\mathrm{n}$ taking values such as 6,8 and 10 can categorize as dipole-dipole, dipole-quadrupole and quadrupole-quadrupole interactions, respectively. Equation 9 is manifested in Figure 13, and the best linear relation with better fitting at $n=8$ demonstrates the mechanism of dipole-quadrupole interactions for energy transfer.

For a dipole-quadrupole interaction, the energy transfer probability $\left(\mathrm{P}_{\mathrm{SA}}\right)$ from a donor to an acceptor is given by the following formula:

$\mathrm{P}_{\mathrm{SA}}(\mathrm{dq})=\frac{3 \times 10^{12} \lambda_{S}^{2} \mathrm{f}_{\mathrm{q}}}{\mathrm{R}^{8} \tau_{\mathrm{S}}} \int \frac{\mathrm{F}_{\mathrm{S}}(\mathrm{E}) \mathrm{F}_{\mathrm{A}}(\mathrm{E})}{\mathrm{E}^{4}} \mathrm{dE}$

where $\lambda_{\mathrm{S}}$ is the emission wavelength of the donor ions, $\tau_{\mathrm{S}}$ is the radiative decay time of the donor, $\mathrm{R}$ is the donor-acceptor average distance. The critical distance $\left(\mathrm{R}_{\mathrm{c}}\right)$ of the energy transfer from the donor to acceptor is defined as the distance for which the probability of transfer equals the probability of radiative emission of the donor, i.e., the distance for which $\mathrm{P}_{\mathrm{SA}} \cdot \tau_{\mathrm{S}}=1$. Further, the donor-acceptor critical distance for energy transfer can be reckoned by the following expression in terms of the electric dipole-quadrupole mechanism (Blass 1969; Dexter 1954):

$R_{c}^{8}=\left(3 \times 10^{12} \lambda_{S}^{2}\right) f_{d} \int \frac{F_{S}(E) F_{A}(E)}{E^{4}} d E$

where $\mathrm{fd} \approx 0.02$ denotes the oscillator strength for the activator $\mathrm{Dy}^{3+}$ ions electric dipole. $\int F_{S}(E) F_{A}(E) d E / E^{4}$ represents shows the spectral overlap amidst the normalized shapes of $\mathrm{Tm}^{3+}$ emission Fs(E) and Dy ${ }^{3+}$ excitation FA(E), and the value is determined to be $0.000123 \mathrm{eV}^{-1}$ in our condition. Ultimately, the critical distance (Rc) of $7.22 \AA$ is confirmed through Eq.(11).

Quantum efficiency (QE) is a very important physical parameter for phosphor materials in the practical application, defined as the ratio of the number of photons emitted to the number of photons absorbed. It can be measured by the integrated sphere (Liu 2011), in accordance to the 
following equation:

$\eta=\frac{E_{i}(\lambda)-(1-A) E_{0}(\lambda)}{L_{e}(\lambda) A}$

where $\mathrm{E}_{\mathrm{i}}(\lambda)$ is the integrated luminescence upon direct excitation, and $\mathrm{E}_{0}(\lambda)$ is the integrated luminescence excited by indirect illumination from the integrated sphere. The value $\mathrm{L}_{e}(\lambda)$ is the integrated excitation profile acquired from the empty integrated sphere (absence of sample). The optical absorbance A can be gained via using the following formula:

$\mathrm{A}=\frac{\mathrm{L}_{0}(\lambda)-\mathrm{L}_{\mathrm{i}}(\lambda)}{\mathrm{L}_{0}(\lambda)}$

where $\mathrm{L}_{0}(\lambda)$ is the integrated excitation profile when the sample is diffusely illuminated by the integrated sphere's surface, and $\mathrm{L}_{\mathrm{i}}(\lambda)$ is the integrated excitation profile when the sample is directly excited by the incident beam ( $\mathrm{Li} 2014$ ). The corresponding value for SBSO:3\% $\mathrm{Tm}^{3+}$, $\mathrm{y} \% \mathrm{Dy}^{3+}(\mathrm{y}=0,3-9)$ phosphors excited at $357 \mathrm{~nm}$ are determined to be about $25.69 \%, 26.77 \%$, $28.53 \%, 31.05 \%$ and $30.26 \%$, respectively.

It is pointed out by Ronda that if the transition from the excited state to the ground state contains both radiative and nonradiative transitions, the transition rate $\left(\mathrm{k}\right.$, in s$\left.{ }^{-1}\right)$ can be written as (Ronda 2008)

$\mathrm{k}=\mathrm{k}_{\mathrm{r}}+\mathrm{k}_{\mathrm{nr}}$

where $\mathrm{k}_{\mathrm{r}}$ is the transition rate of only the emission process, and $\mathrm{k}_{\mathrm{nr}}$ is the rate of the nonradiative process. The existence of the nonradiative process will increase the transition rate, namely, a shorter decay time ( $\tau$, equals $1 / \mathrm{k}$ ). If the decay time $\left(\tau_{0}\right)$ without nonradiative transitions is known, the $\mathrm{QE}$ of the phosphor can be expressed as

$\mathrm{QE}=\tau / \tau_{0}$

from the equation, it can be known that the change of QE should be consistent with the decay time, and the above results (decay time and QE) for our phosphors also confirm the conclusion. Therefore, the decrease of $\mathrm{QE}$ values for phosphors may be due to the increase of the nonradiation rate.

\subsection{Luminescence and energy transfer characteristics of SBSO:Dy ${ }^{3+}, \mathrm{Eu}^{3+}$}

As we all know, the high efficiency red emission corresponding to the typical $4 \mathrm{f}-4 \mathrm{f}$ parity-forbidden is the characteristic luminescence for $\mathrm{Eu}^{3+}$ ions. It is considerable that $\mathrm{Eu}^{3+}$ ions are applied in the $\mathrm{Dy}^{3+}$ single-doped phosphor for supplying plentiful red constituent to implement 
the multicolor emission in accordance with the energy transfer of $\mathrm{Dy}^{3+} \rightarrow \mathrm{Eu}^{3+}$. The photoluminescence excitation and emission spectra of $\mathrm{Eu}^{3+}$-doped SBSO samples are exhibited in Figure 14a. The excitation spectrum through the typical emission $(611 \mathrm{~nm})$ monitoring comprises a sharp line at $392 \mathrm{~nm}$ deriving from the transition ${ }^{7} \mathrm{~F}_{0} \rightarrow{ }^{5} \mathrm{~L}_{6}$ as well as a broad absorption band from 250 to $315 \mathrm{~nm}$ contributed principally by the $\mathrm{Eu}^{3+}-\mathrm{O}^{2-}$ and matrix charge transfer. In addition, there is a sharp absorption line appearing at $465 \mathrm{~nm}$, ascribed to the energy level for ${ }^{7} \mathrm{~F}_{0} \rightarrow{ }^{5} \mathrm{D}_{2}$, that is the hypersensitive transition of $\mathrm{Eu}^{3+}$ ions. According to the Judd-Ofelt (JO) theory (Shanmuga Sundari et al. 2010), the hypersensitive transition follows the selection rule $|\Delta \mathrm{J}| \leqslant 6,|\Delta \mathrm{L}| \leqslant 2, \Delta \mathrm{S}=0$ and is related to parameter $\Omega_{2}$. In the present work, parameter $\Omega_{2}$ indicates the covalence of $\mathrm{Eu}-\mathrm{O}$ bond and the asymmetry around $\mathrm{Eu}^{3+}$ site. The parameter $\Omega_{2}$ of hypersensitive transition is of special sensitivity to surrounding environment and related to the linear term of crystal field action. That is, the hypersensitive transition can occur only when the local symmetry makes the crystal field interaction having a linear term. Thus, the hypersensitive transition absorption (465 nm) in SBSO: $\mathrm{Eu}^{3+}$ indicate that $\mathrm{Eu}^{3+}$ is in the symmetry of $\mathrm{C}_{20}$ point group with linear term crystal field in the orthorhombic symmetry structure.

In the section of 500-750 nm (Figure 14a), the emission spectrum upon stimulation of $392 \mathrm{~nm}$ exhibits several emission bands at $577,590,611$ and $649 \mathrm{~nm}$ corresponding to the typical ${ }^{5} \mathrm{D}_{0} \rightarrow{ }^{7} \mathrm{~F}_{0}$, ${ }^{7} \mathrm{~F}_{1},{ }^{7} \mathrm{~F}_{2}$ and ${ }^{7} \mathrm{~F}_{3}$ transitions for $\mathrm{Eu}^{3+}$ ions. The special emission transition of ${ }^{5} \mathrm{D}_{0} \rightarrow{ }^{7} \mathrm{~F}_{0}(\mathrm{~J}=0)$ at 577 $\mathrm{nm}$ disconfirms the selection rule (Judd-Ofelt theory), belonging to the parity-forbidden transition. But the crystal field interaction having a linear term of $C_{s}, C_{n}$ or $C_{n v}$ point group symmetry site can prompt the emission for ${ }^{5} \mathrm{D}_{0} \rightarrow{ }^{7} \mathrm{~F}_{0}$. The appearance of ${ }^{5} \mathrm{D}_{0} \rightarrow{ }^{7} \mathrm{~F}_{0}$ in SBSO:Eu ${ }^{3+}$ confirms the conclusion for the hypersensitive transition absorption again. It is observed that the emission transition for ${ }^{5} \mathrm{D}_{0} \rightarrow{ }^{7} \mathrm{~F}_{2}$ follows the selection rule $|\Delta \mathrm{J}| \leqslant 6$ which comes under electric dipole induced in nature and remaining transition of ${ }^{5} \mathrm{D}_{0} \rightarrow{ }^{7} \mathrm{~F}_{1}$ which obey the selection rule $|\Delta \mathrm{J}|=0, \pm 1$ comes under magnetic dipole in nature. Generally, the parity of all states in $4 \mathrm{f}^{\mathrm{n}}$ configuration of rare earth ions is the same. According to the parity selection rule of rare earth ions transition, it is known that the electric dipole is parity-forbidden and the magnetic dipole is parity-allowed in $4 \mathrm{f}^{\mathrm{n}}$ configuration. When the sites of $\mathrm{Eu}^{3+}$ ions are of inversion symmetry, only magnetic dipole allowed transition for ${ }^{5} \mathrm{D}_{0} \rightarrow{ }^{7} \mathrm{~F}_{1}$ appears due to the crystal field without odd term. If the sites are lack of inversion symmetry, the opposite parity is mixed into $4 \mathrm{f}^{\mathrm{n}}$ configuration, which makes the 
rule of parity selection relaxed because of the odd term of crystal field. At this time, the electric dipole transition of ${ }^{5} \mathrm{D}_{0} \rightarrow{ }^{7} \mathrm{~F}_{2}$ dominates. In the present work, it can be found that the featured transition of ${ }^{5} \mathrm{D}_{0} \rightarrow{ }^{7} \mathrm{~F}_{2}$ for electric dipole at $611 \mathrm{~nm}$ is superior to other transitions prominently (the magnetic dipole transition of ${ }^{5} \mathrm{D}_{0} \rightarrow{ }^{7} \mathrm{~F}_{1}$ at $590 \mathrm{~nm}$ ), intimating that the sites of $\mathrm{Eu}^{3+}$ ions is lack of inversion symmetry. This also confirms the conclusion obtained from the emission spectrum of $\mathrm{Dy}^{3+}$ ion. The emission spectra of $\mathrm{SBSO}: \mathrm{x} \% \mathrm{Eu}^{3+}(\mathrm{x}=1-13)$ with diverse molarity of $\mathrm{Eu}^{3+}$ are revealed in Figure 14b. The optimal emission concentration for $\mathrm{Eu}^{3+}$ ions is confirmed to be 9 mol\% in SBSO host.

As contrasted in Figure 15, the excitation spectrum for SBSO:7\% $\mathrm{Dy}^{3+}, 9 \% \mathrm{Eu}^{3+}$ via the 611 $\mathrm{nm}$ emission $\left(\mathrm{Eu}^{3+}\right)$ monitoring is constituted by the composition of manifest $\mathrm{Eu}^{3+}$ and $\mathrm{Dy}^{3+}$ excitation bands, which substantiates the energy migration for $\mathrm{Dy}^{3+} \rightarrow \mathrm{Eu}^{3+}$ preliminarily.

A series of SBSO:7\% $\mathrm{Dy}^{3+}, \mathrm{y} \% \mathrm{Eu}^{3+}(\mathrm{y}=0,1,3,5,7,9,11,13)$ were prepared and Figure 16 displays the PL spectra corresponding to the as-synthesized samples with a $349 \mathrm{~nm}$ excitation source. The emission spectra exhibit not only the characteristic yellow emissions of $\mathrm{Dy}^{3+}$ at 487 and $576 \mathrm{~nm}$ but also the red emitting peaks of $\mathrm{Eu}^{3+}$ around $611 \mathrm{~nm}$. Although the $\mathrm{Dy}^{3+}$ ion concentration remains constant at 0.07 , the feature emission $\left(\mathrm{Dy}^{3+}\right)$ weakens along with increasing $\mathrm{Eu}^{3+}$ content, in the meantime the red luminescence $\left(\mathrm{Eu}^{3+}\right)$ rises until the concentration quenching at $\mathrm{y}=9$. The above exploration in the luminescence for $\mathrm{Dy}^{3+}$ and $\mathrm{Eu}^{3+}$ ions forcefully demonstrate the behavior in the transfer of energy from $\mathrm{Dy}^{3+}$ to $\mathrm{Eu}^{3+}$.

The decay luminescence for $\mathrm{Dy}^{3+}$ ion in SBSO:7\% $\mathrm{Dy}^{3+}, \mathrm{y} \% \mathrm{Eu}^{3+}(\mathrm{y}=0,1-13)$ upon the stimulation at $349 \mathrm{~nm}$ with $576 \mathrm{~nm}$ emission monitoring were recorded and presented in Figure 17. All the decay behaviors show a precise fit with Eq.(5). According to Eq.(5) and (6), the characteristic lifetimes of $\mathrm{Dy}^{3+}$ emission were reckoned to be $0.67,0.60,0.56,0.53,0.49$ and 0.42 ms for $\mathrm{Eu}^{3+}$ concentrations as $0,3 \%, 5 \%, 7 \%, 9 \%$ and $11 \%$, respectively. It reflects the phenomenon that the decay time of $\mathrm{Dy}^{3+}$ monotonically decreases with the promoting amount of $\mathrm{Eu}^{3+}$ ions, which provides convincing evidence for the energy transfer of $\mathrm{Dy}^{3+} \rightarrow \mathrm{Eu}^{3+}$. The ET efficiencies were estimated employing Eq.(7) and the relation curve versus $\mathrm{Eu}^{3+}$ molarity is plotted in Figure 18. The $\eta_{\mathrm{T}}$ value enlarges constantly in the wake of $\mathrm{Eu}^{3+}$ concentrations rising and approximately is $82 \%$ at $\mathrm{y}=13$.

It can be estimated that $R_{\mathrm{Dy}-\mathrm{Eu}}$ is greater than $5 \AA$ by Eq.(2), therefore the mechanism of 
energy transfer for $\mathrm{Dy}^{3+} \rightarrow \mathrm{Eu}^{3+}$ in SBSO via electronic exchange interaction is excluded. Similarly, based on the relevant theory of multipolar interaction, the most plausible energy migration process for $\mathrm{Dy}^{3+} \rightarrow \mathrm{Eu}^{3+}$ was analyzed by Eq.(9). The linear relationship of $\left(\mathrm{I}_{\mathrm{so}} / \mathrm{I}_{\mathrm{s}}\right) \propto \mathrm{C}^{\mathrm{n} / 3}$ are represented in Figure 19 , and the best fitting factor $\mathrm{R}^{2}=0.8832$ at $\mathrm{n}=6$ implied a dipole-dipole interaction for the energy transfer mechanism. For a dipole-dipole interaction, the donor-acceptor critical distance for energy transfer can be reckoned by the following expression (Blass 1969; Dexter 1954):

$\mathrm{R}_{\mathrm{c}}^{6}=\left(3 \times 10^{12}\right) \mathrm{f}_{\mathrm{d}} \int \frac{\mathrm{F}_{\mathrm{S}}(\mathrm{E}) \mathrm{F}_{\mathrm{A}}(\mathrm{E})}{\mathrm{E}^{4}} \mathrm{dE}$

Thereby, the critical distance ( $\left.\mathrm{R}_{\mathrm{Dy}-\mathrm{Eu}}\right)$ is computed as $10.79 \AA$ using Eq.(16).

The energy level transition of ET mechanism in $\mathrm{Tm}^{3+} / \mathrm{Dy}^{3+} / \mathrm{Eu}^{3+}$ co-doped SBSO system was investigated according to the schematic model as shown in Figure 20. Under the irradiation at 357 $\mathrm{nm}$ the electrons located at the ground state ${ }^{3} \mathrm{H}_{6}$ are promoted to the emission level ${ }^{1} \mathrm{D}_{2}$ and then part of the stimulated electrons return to the lower state $\left({ }^{1} \mathrm{D}_{2} \rightarrow{ }^{3} \mathrm{~F}_{4}\right)$ in $\mathrm{Tm}^{3+}$ ions, and emit a certain wavelength of light. While, the difference between the ${ }^{1} \mathrm{D}_{2}$ level of $\mathrm{Tm}^{3+}$ and the ${ }^{4} \mathrm{I}_{11 / 2}$ level of $\mathrm{Dy}^{3+}$ is not significant, another part of the stimulated electrons can transfer energy to $\mathrm{Dy}^{3+}$ in a non-radiative form, namely ET1. Meanwhile, a portion of $\mathrm{Tm}^{3+}$ emission is also received by $\mathrm{Dy}^{3+}$ ion, namely ET2, which arouses the electrons transition for ${ }^{6} \mathrm{H}_{15 / 2} \rightarrow{ }^{4} \mathrm{P}_{7 / 2}\left(\mathrm{Dy}^{3+}\right)$, since the manifest overlap emerging in the spectra of $\mathrm{Dy}^{3+}$ and $\mathrm{Tm}^{3+}$. The activated electrons in the ${ }^{6} \mathrm{P}_{7 / 2}$ and ${ }^{4} \mathrm{I}_{11 / 2}$ level of $\mathrm{Dy}^{3+}$ undergo non-radiative relaxation, liberate to the ${ }^{4} \mathrm{~F}_{9 / 2}$ level and then display a radiative release to the ${ }^{6} \mathrm{H}_{13 / 2}$ and ${ }^{6} \mathrm{H}_{15 / 2}$ levels under the combined actions of ET1 and ET2, giving rise to the typical yellow emission of $\mathrm{Dy}^{3+}$ at 488 and $576 \mathrm{~nm}$. The energy level transition mechanism between $\mathrm{Dy}^{3+}$ and $\mathrm{Eu}^{3+}$ only belongs to ET1. Some energy for ${ }^{4} \mathrm{~F}_{9 / 2}$ level $\left(\mathrm{Dy}^{3+}\right)$ is taken in the ${ }^{5} \mathrm{~L}_{6}$ level $\left(\mathrm{Eu}^{3+}\right)$ through the modality of ET1, and the activated electrons at the emission level ${ }^{5} \mathrm{~L}_{6}$ liberate to the lower energetic state ${ }^{5} \mathrm{D}_{0}$ by non-radiative relaxation. Further, a radiative release to the ground state ${ }^{7} \mathrm{~F}_{0,1,2,3}$, induces the feature emissions at 579, 591, 611 and $652 \mathrm{~nm}$ for $\mathrm{Eu}^{3+}$ ion, respectively. It can be known from the above analysis that the cross relaxation occurs in the process of energy transfer for $\mathrm{Tm}^{3+} \rightarrow \mathrm{Dy}^{3+}$ and $\mathrm{Dy}^{3+} \rightarrow \mathrm{Eu}^{3+}$, that is, the transition of $\quad{ }^{1} \mathrm{D}_{2} \quad\left(\mathrm{Tm}^{3+}\right) \rightarrow{ }^{4} \mathrm{~F}_{9 / 2} \quad\left(\mathrm{Dy}^{3+}\right) \rightarrow{ }^{6} \mathrm{H}_{15 / 2} \quad\left(\mathrm{Dy}^{3+}\right)$ and $\left.{ }^{6} \mathrm{P}_{7 / 2} \quad\left(\mathrm{Dy}^{3+}\right) \rightarrow{ }^{4} \mathrm{~F}_{9 / 2} \quad\left(\mathrm{Dy}^{3+}\right)\right|^{5} \mathrm{D}_{0}$ $\left(\mathrm{Eu}^{3+}\right) \rightarrow{ }^{7} \mathrm{~F}_{\mathrm{J}}\left(\mathrm{Eu}^{3+}\right)$. With the increasing of luminescent center concentration, the distance decreases and the interaction increases, which eventually leads to the raising of lattice vibration frequency. 
In this case, the energy donor relaxes from the higher excited energy level to the lower excited level and then returns to the ground state in form of radiation to make the emission of light. Although this process leads to the emission quenching of high energy level, it also increases the aggregation number at low excited energy level. This is beneficial to the emission of the main energy level, so as to enhance the luminous intensity. Thus, the cross relaxation process in the energy transfer for $\mathrm{Tm}^{3+} \rightarrow \mathrm{Dy}^{3+}$ and $\mathrm{Dy}^{3+} \rightarrow \mathrm{Eu}^{3+}$ improves the luminous efficiency of energy acceptor $\mathrm{Dy}^{3+}$ and $\mathrm{Eu}^{3+}$ ions. However, the concentration quenching will occur when constant is further enhanced (beyond the critical distance), as discussion in ninth paragraph.

The Commission International de L'Eclairage (CIE) chromaticity coordinates of SBSO: $\mathrm{Tm}^{3+} / \mathrm{Dy}^{3+} / \mathrm{Eu}^{3+}$ specimen subjected to ultraviolet light excitation are disclosed in Figure 21 and Table 4. For SBSO:7\% $\mathrm{Dy}^{3+}, \mathrm{y} \% \mathrm{Eu}^{3+}$ system, as aggrandizing the value of $\mathrm{y}(0-0.13)$, the color coordinates are converted piecemeal from $(0.372,0.401)$ to $(0.553,0.395)$ in accordance with the color tones from yellow to red. Based on the results, the SBSO:7\% $\mathrm{Dy}^{3+}, \mathrm{y} \% \mathrm{Eu}^{3+}$ phosphors can achieve multicolor emission from yellow to red by modulating the molarity ratio of doping $\mathrm{Dy}^{3+} / \mathrm{Eu}^{3+}$ ions for its potential adhibitions in solid-state lighting. In addition, the SBSO:3\% $\mathrm{Tm}^{3+}$ phosphor excited by $357 \mathrm{~nm}$ can exhibit bright blue emission, and then the emitting color transferred from blue to white region after introducing $\mathrm{Dy}^{3+}$ ions. Furthermore, complete emission of white light is obtained when the doping levels of $\mathrm{Tm}^{3+}$ and $\mathrm{Dy}^{3+}$ in the samples are appropriately changed, which shall be potentially applied in white LEDs. Generally, particle size of phosphors is considered as an important parameter in the application of white LEDs. The particle size distributions of SBSO:3\% $\mathrm{Tm}^{3+}, 7 \% \mathrm{Dy}^{3+}$ and SBSO:7\% $\mathrm{Dy}^{3+}, 9 \% \mathrm{Eu}^{3+}$ phosphors were measured and exhibited in Figure 22. The average diameters are calculated to be 7.89 and $7.43 \mu \mathrm{m}$, respectively, conforming to the requirement of application.

\section{Conclusion}

On the whole, a sort of original emission-tunable single-component SBSO: $\mathrm{Tm}^{3+} / \mathrm{Dy}^{3+} / \mathrm{Eu}^{3+}$ phosphor is prepared by high temperature solid state route and their luminescence characteristics were minutely studied. The steady-state and transient-state photoluminescence spectra/decay behaviors indicated the existence of the energy migration for $\mathrm{Tm}^{3+} \rightarrow \mathrm{Dy}^{3+}$ and $\mathrm{Dy}^{3+} \rightarrow \mathrm{Eu}^{3+}$ in the SBSO host and the ET efficiency can reach about $80 \%$. While the efficient energy transfer emerges through the dipole-quadrupole mechanism for $\mathrm{Tm}^{3+} \rightarrow \mathrm{Dy}^{3+}$ and the 
dipole-dipole interaction for $\mathrm{Dy}^{3+} \rightarrow \mathrm{Eu}^{3+}$, and the critical distance $\left(\mathrm{R}_{\mathrm{Tm}-\mathrm{Dy}}\right.$ and $\left.\mathrm{R}_{\mathrm{Dy}-\mathrm{Eu}}\right)$ is estimated to be $7.22 \AA$ And $10.79 \AA$, respectively. Furthermore, the tunable yellow-to-red emission can be achieved in SBSO:Dy ${ }^{3+}, \mathrm{Eu}^{3+}$ systems and $\mathrm{Tm}^{3+}$, $\mathrm{Dy}^{3+}$ co-doping samples can emit light entirely in the white region by the appropriate changeable doping molar quantity of $\mathrm{Dy}^{3+} / \mathrm{Eu}^{3+}$ and $\mathrm{Tm}^{3+} / \mathrm{Dy}^{3+}$ according to the energy migration in SBSO. These outstanding features make SBSO: $\mathrm{Tm}^{3+} / \mathrm{Dy}^{3+} / \mathrm{Eu}^{3+}$ promising w-LED phosphors for its potential adhibitions in solid-state optical applications.

\section{Acknowledgments}

This funding for the current research is received from the Major Program for the Field (No: 2016009) and PhD Start-Up Funding (No: 2017001), Jilin Institute of Chemical Technology. 


\section{References}

Chen, J., Liu, Y.A., Fang, M.H., Huang, Z.H.: Luminescence properties and energy transfer of Eu/Mn-coactivated $\mathrm{Mg}_{2} \mathrm{Al}_{4} \mathrm{Si}_{5} \mathrm{O}_{18}$ as a potential phosphor for white-light LEDs. Inorg. Chem. 53, 11396-11403 (2014)

Bharat, L.K., Du, P., Yu, J.S.: Long-wave UVA radiation excited warm white-light emitting $\mathrm{NaGdTiO}_{4}: \mathrm{Tm}^{3+} / \mathrm{Dy}^{3+} / \mathrm{Eu}^{3+}$ ions tri-doped phosphors: synthesis, energy transfer and color tunable properties. J. Alloys. Compd. 666, 270-278 (2016)

Shang, M.M., Li, C.X., Lin, J.: How to produce white light in a single-phase host. J. Chem. Soc. Rev. 43, 1372-1386 (2014)

Liu, L.F., Wang, G.G., Yang, Q., Yan, B., Zhang, H.Y., Han, J.C.: Highly thermally stable single-component warm-white-emitting ZANP glass: synthesis, luminescence, energy transfer, and color tenability. Ceram. Int. 10, 11693-11701 (2018)

Wang, B., Lin, H., Huang, F., Xu, J., Chen, H., Lin, Z.B., Wang, Y.S.: Non-rare-earth $\mathrm{BaMgAl}_{10-2 \mathrm{x}} \mathrm{O}_{17}: \mathrm{xMn}^{4+}, \mathrm{xMg}^{2+}:$ an arrow-band red phosphor for use as a high-power warm w-LED. Chem. Mater. 28, 3515-3524 (2016)

Ye, S., Xiao, F., Pan, Y.X., Ma, Y.Y., Zhang, Q.Y.: Phosphors in phosphor-converted white light-emitting diodes: recent advances in materials, techniques and properties. Mat. Sci. Eng. R-Rep. 71, 1-34 (2010)

Smet, P.F., Parmentier, A.B., Poelman, D.: Selecting conversion phosphors for white light-emitting diodes. J. Electrochem. Soc. 158, R37-R54 (2011)

Liu, Y., Liu, G.X., Wang, J.X., Dong, X.T., Yu, W.S.: Single-component and warm-white-emitting phosphor $\mathrm{NaGd}\left(\mathrm{WO}_{4}\right)_{2}: \mathrm{Tm}^{3+}, \mathrm{Dy}^{3+}, \mathrm{Eu}^{3+}$ : synthesis, luminescence, energy transfer, and tunable color. Inorg. Chem. 53, 11457-11466 (2014)

Luwang, M.N., Ningthoujam, R.S., Srivastava, S.K., Vatsa ,R.K.: Preparation of white light emitting $\mathrm{YVO}_{4}: \mathrm{Ln}^{3+}$ and silica-coated $\mathrm{YVO}_{4}: \mathrm{Ln}^{3+}\left(\mathrm{Ln}^{3+}=\mathrm{Eu}^{3+}, \mathrm{Dy}^{3+}, \mathrm{Tm}^{3+}\right)$ nanoparticles by $\mathrm{CTAB} / \mathrm{n}$-butanol/hexane/water microemulsion route: energy transfer and site symmetry studies. J. Mater. Chem. 21, 5326-5337 (2011)

Zhu, C., Yang, Y., Liang, X., Yuan, S., Chen, G.: Rare earth ions doped full-color luminescence glasses for white LED. J. Lumin. 126, 707-710 (2007) 
Patnam, H., Hussain, S.K., Bharat, L.K., Yu, J.S.: Near-ultraviolet excited $\mathrm{Tm}^{3+}$ and $\mathrm{Dy}^{3+}$ ions co-doped barium lanthanum silica oxide phosphors for white-light applications. J. Alloys. Compd. 780, 846-855 (2019)

Zhu, H.M., Lin, C.C., Luo, W.Q., Shu, S.T., Liu, Z.G., Liu, Y.S., Kong, J.T., Ma E., Cao, Y.G., Liu, R.S., Chen, X.Y.: Highly efficient non-rare-earth red emitting phosphor for warm white light-emitting diodes. Nat. Commun. 5, 4312 (2014)

Chen, B.J., Shen, L.F., Pun, E.Y.B., Lin, H.: Sm-doped germinate glass channel waveguide as light source for minimally invasive photodynamic therapy surgery. Opt. Express. 20, 879-889 (2012)

Song, K.X., Zhang, J.X., Liu, Y.F., Zhang, C.H., Jiang, J., Jiang, H.C., Qin, H.B.: Red-emitting phosphor $\mathrm{Ba}_{9} \mathrm{Lu}_{2} \mathrm{Si}_{6} \mathrm{O}_{24}: \mathrm{Ce}^{3+}, \mathrm{Mn}^{2+}$ with enhanced energy transfer via self-charge compensation. J. Phys. Chem. C. 119, 24558-24563 (2015)

Setlur, A.: Phosphors for LED-based solid-state lighting. Electrochem. Soc. Interface.18, 32-36 (2009)

Hu, J., Gong, X.H., Chen, Y.J., Huang, J.H., Lin, Y.F., Luo, Z.D., Huang, Y.D.: Tunable luminescence of $\mathrm{Dy}^{3+}$ single-doped and $\mathrm{Dy}^{3+} / \mathrm{Tm}^{3+}$ co-doped tungsten borate glasses. Opt. Mater. 38, 108-112 (2014)

Pavitra, E., Raju, G.S.R., Park, W.: Concentration and penetration depth dependent tunable emissions from $\mathrm{Eu}^{3+}$ co-doped $\mathrm{SrY}_{2} \mathrm{O}_{4}: \mathrm{Dy}^{3+}$ nanocrystalline phosphor. New. J. Chem. 38, 163-169 (2013)

Huang, A.J., Yang, Z.W., Yu, C.Y., Chai, Z.Z.: Tunable and white light emission of a single-phased $\mathrm{Ba}_{2} \mathrm{Y}\left(\mathrm{BO}_{3}\right)_{2} \mathrm{Cl}: \mathrm{Bi}^{3+}, \mathrm{Eu}^{3+}$ phosphor by energy transfer for ultraviolet converted white LEDs. J. Phys. Chem. C. 121, 5267-5276 (2017)

Zhao, L., Meng, D.D., Li, Y.Y., Zhang, Y., Wang, H.Q.: Tunable emitting phosphors $\mathrm{K}_{3} \mathrm{Gd}\left(\mathrm{PO}_{4}\right)_{2}: \mathrm{Tm}^{3+}-\mathrm{Dy}^{3+}$ for light emitting diodes and field emission displays. J. Alloys. Compd. 728, 564-570 (2017)

Li, W.J., Zhang, W.T., Li, W.W., Gan, Y., Zhang ,P.C.: Synthesis and luminescence properties of $\mathrm{Eu}^{3+}$ co-doped $\mathrm{NaBi}\left(\mathrm{MoO}_{4}\right)_{2}: \mathrm{Dy}^{3+}$ phosphors for white light-emitting diodes. J. Mater. Sci-Mater. Electron. 30, 658-666 (2019)

Mondal, K., Manam, J.: Investigation of photoluminescence properties, thermal stability, energy 
transfer mechanisms and quantum efficiency of $\mathrm{Ca}_{2} \mathrm{ZnSi}_{2} \mathrm{O}_{7}: \mathrm{Dy}^{3+}, \mathrm{Eu}^{3+}$ phosphors. J. Lumin. 195, 259-270 (2018)

Chang, C.K., Chen, T.M.: $\mathrm{Sr}_{3} \mathrm{~B}_{2} \mathrm{O}_{6}: \mathrm{Ce}^{3+}, \mathrm{Eu}^{2+}$ : A potential single-phased white-emitting borate phosphor for ultraviolet light-emitting diodes. Appl. Phys. Lett. 91, 081902 (2007)

Wang, L.S., Wang, Y.H.: Luminescent properties of $\mathrm{Eu}^{3+}$-activated $\mathrm{Sr}_{3} \mathrm{~B}_{2} \mathrm{SiO}_{8}$ : A red-emitting phosphor for white light-emitting diodes. J. Lumin. 131, 1479-1481 (2011)

Sabikoglu, I.: Synthesis of Eu and Dy doped $\mathrm{Sr}_{3} \mathrm{~B}_{2} \mathrm{SiO}_{8}$ using solid state reaction and investigation of radio and photoluminescence properties of these materials. J. Alloys. Compd. 556, $135-138$ (2013)

Sun, J.F., Ding, D.B., Sun, J.Y.: Synthesis and photoluminescence properties of a novel reddish orange-emitting $\mathrm{Sm}^{3+}$-doped strontium borosilicate phosphor. Opt. Mater. 58, 188-195 (2016)

Blass, G.: Handbook on the Physics and Chemistry of Rare Earths. North-Holland Publishing Company. 237 (1979)

Blass, G.: Energy transfer in oxidic phosphors. Philips. Res. Rep. 24, 131-144 (1969)

Zhao, M., Liao, H., Ning, L., Zhang, Q., Liu, Q., Xia, Z.: Next generation narrow-band green-emitting $\mathrm{RbLi}\left(\mathrm{Li}_{3} \mathrm{SiO}_{4}\right)_{2}: \mathrm{Eu}^{2+}$ phosphor for backlight display application. Adv. Mater. 30, 1802489 (2018)

Li, Y.Y., Shi, Y.R., Zhu, G., Wu, Q.S., Li, H., Wang, X.C., Wang, Q., Wang, Y.H.: A single-component white-emitting $\mathrm{CaSr}_{2} \mathrm{Al}_{2} \mathrm{O}_{6}: \mathrm{Ce}^{3+}, \mathrm{Li}^{+}, \mathrm{Mn}^{2+}$ phosphor via energy transfer. Inorg. Chem. 53, 7668-7675 (2014)

Wang, M.S., Guo, S.P., Li, Y., Cai, L.Z., Zou, J.P., Xu, G., Zhou, W.W., Zheng, F.K., Guo, G.C.: A direct white-light-emitting metal-organic framework with tunable yellow-to-white photoluminescence by variation of excitation light. J. Am. Chem. Soc. 131, 13572-13573 (2009)

Dexter, D.L., Schulman, J.H.: Theory of concentration quenching in inorganic phosphors. J. Chem. Phys. 22, 1063-1070 (1954)

Dexter, D.L. J. Chem. Phys. 21, 836-850 (1954)

Reisfeld, R., Lieblich-Soffer, N.: Energy transfer from $\mathrm{UO}_{2}{ }^{2+}$ to $\mathrm{Sm}^{3+}$ in phosphate glass. J. Solid. State. Chem. 28, 391-395 (1979) 
Liu, W.R., Huang, C.H., Wu, C.P., Chiu, Y.C., Yeh, Y.T., Chen, T.M.: High efficiency and high color purity blue-emitting $\mathrm{NaSrBO}_{3}: \mathrm{Ce}^{3+}$ phosphor for near-UV light-emitting diodes J. Mater. Chem. 21, 6869-6874 (2011)

Li, K., Shang, M.M., Geng, D.L., Lian, H.Z., Lin, J.: Synthesis, Luminescence, and energy-transfer properties of $\beta-\mathrm{Na}_{2} \mathrm{Ca}_{4}\left(\mathrm{PO}_{4}\right)_{2}\left(\mathrm{SiO}_{4}\right): \mathrm{A} \quad\left(\mathrm{A}=\mathrm{Eu}^{2+}, \quad \mathrm{Dy}^{3+}, \quad \mathrm{Ce}^{3+} / \mathrm{Tb}^{3+}\right)$ phosphors. Inorg. Chem. 53, 6743-6751 (2014)

Ronda, C., Ed.: Luminescence-from Theory to Applications. Wiley-VCH Verlag Cmbh \& Co. KGaA: Weinheim. (2008)

Shanmuga Sundari, S., Marimuthu, K., Sivaraman, M., Surendra Banu, S.: Composition dependent structural and optical properties of $\mathrm{Sm}^{3+}$-doped sodium borate and sodium fluoroborate glasses. J. Lumin. 130, 1313-1319 (2010) 


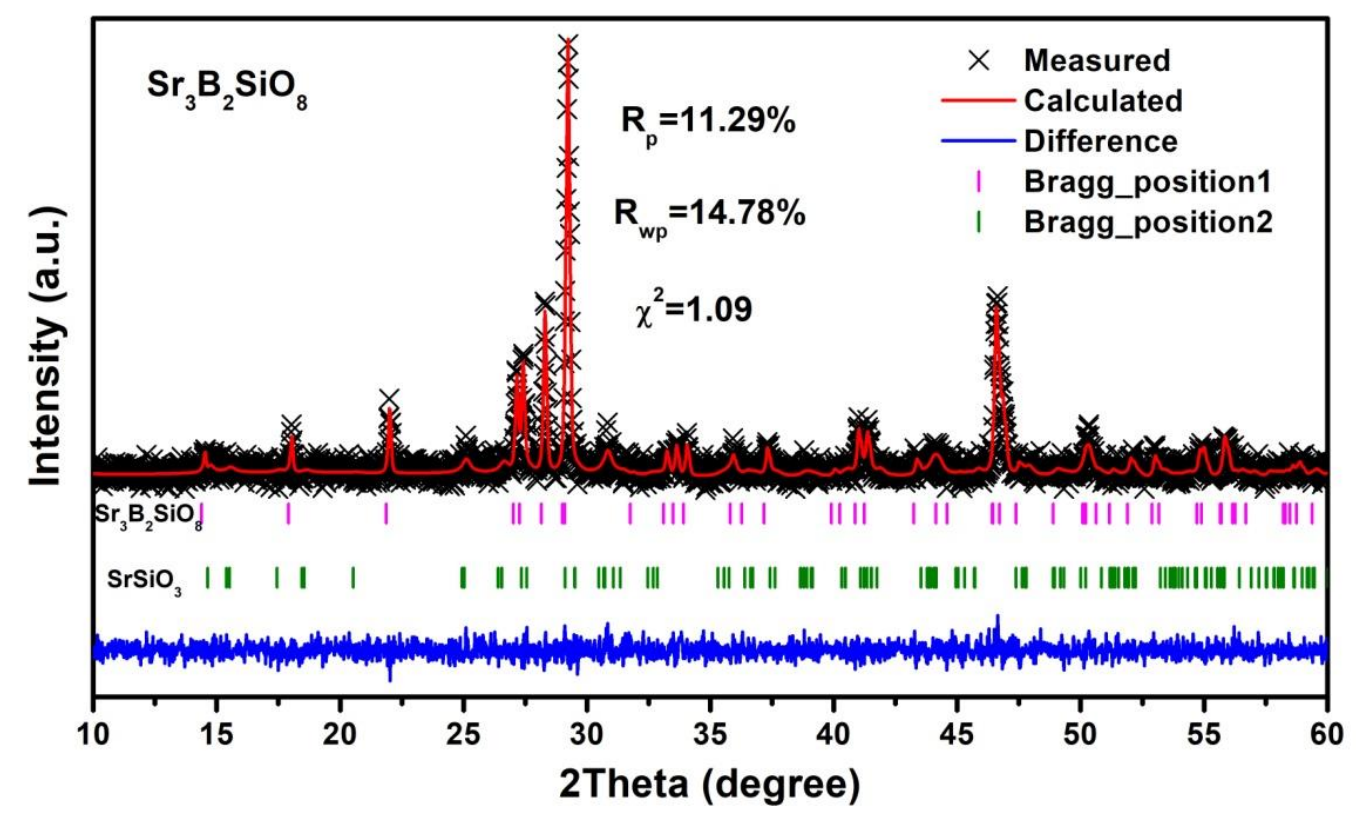

Fig. 1 XRD pattern for the Rietveld refinement of $\mathrm{Sr}_{3} \mathrm{~B}_{2} \mathrm{SiO}_{8}$ 
Table 1 Crystal structural data of SBSO as the results of Rietveld refinement

\begin{tabular}{cc}
\hline \multicolumn{2}{c}{ Crystal structural and lattice parameters of SBSO } \\
\hline Formula & $\mathrm{Sr}_{3} \mathrm{~B}_{2} \mathrm{SiO}_{8}$ \\
\hline Crystal system & orthorhombic \\
\hline Space group & Pnma \\
\hline $\mathbf{a} / \AA$ & 12.3025 \\
\hline $\mathbf{b} / \AA$ & 3.9061 \\
\hline $\mathbf{c} / \AA$ & 5.4068 \\
\hline $\boldsymbol{\alpha} / \mathbf{d e g}$ & 90 \\
\hline $\boldsymbol{\beta} / \mathbf{d e g}$ & 90 \\
\hline$\gamma / \mathbf{d e g}$ & 90 \\
\hline $\mathbf{V} / \AA^{\mathbf{3}}$ & 259.82 \\
\hline $\mathbf{R}_{\mathbf{p}} / \%$ & 11.29 \\
\hline $\mathbf{R}_{\mathbf{w p}} / \%$ & 14.78 \\
\hline$\chi^{\mathbf{2}}$ & 1.09 \\
\hline
\end{tabular}

Table 2 Atomic Coordinates and Atomic Occupancy Ratio of SBSO Obtained by the Rietveld Refinement

\begin{tabular}{ccccc}
\hline Atom & $\mathbf{x}$ & $\mathbf{y}$ & $\mathbf{z}$ & SOF \\
\hline Sr1 & 0.1470 & 0.75 & 0.9058 & 1.0 \\
\hline B1 & 0.4136 & 0.75 & 0.9342 & 0.67 \\
\hline Si1 & 0.4136 & 0.75 & 0.9342 & 0.33 \\
\hline O1 & 0.4213 & 0.75 & 0.6732 & 0.48 \\
\hline O2 & 0.5215 & 0.9015 & 0.9351 & 0.71 \\
\hline O3 & 0.3440 & 0.75 & 0.1154 & 0.29 \\
\hline O4 & 0.3655 & 0.75 & 0.667 & 0.60 \\
\hline O5 & 0.3121 & 0.75 & 0.0821 & 0.90 \\
\hline
\end{tabular}



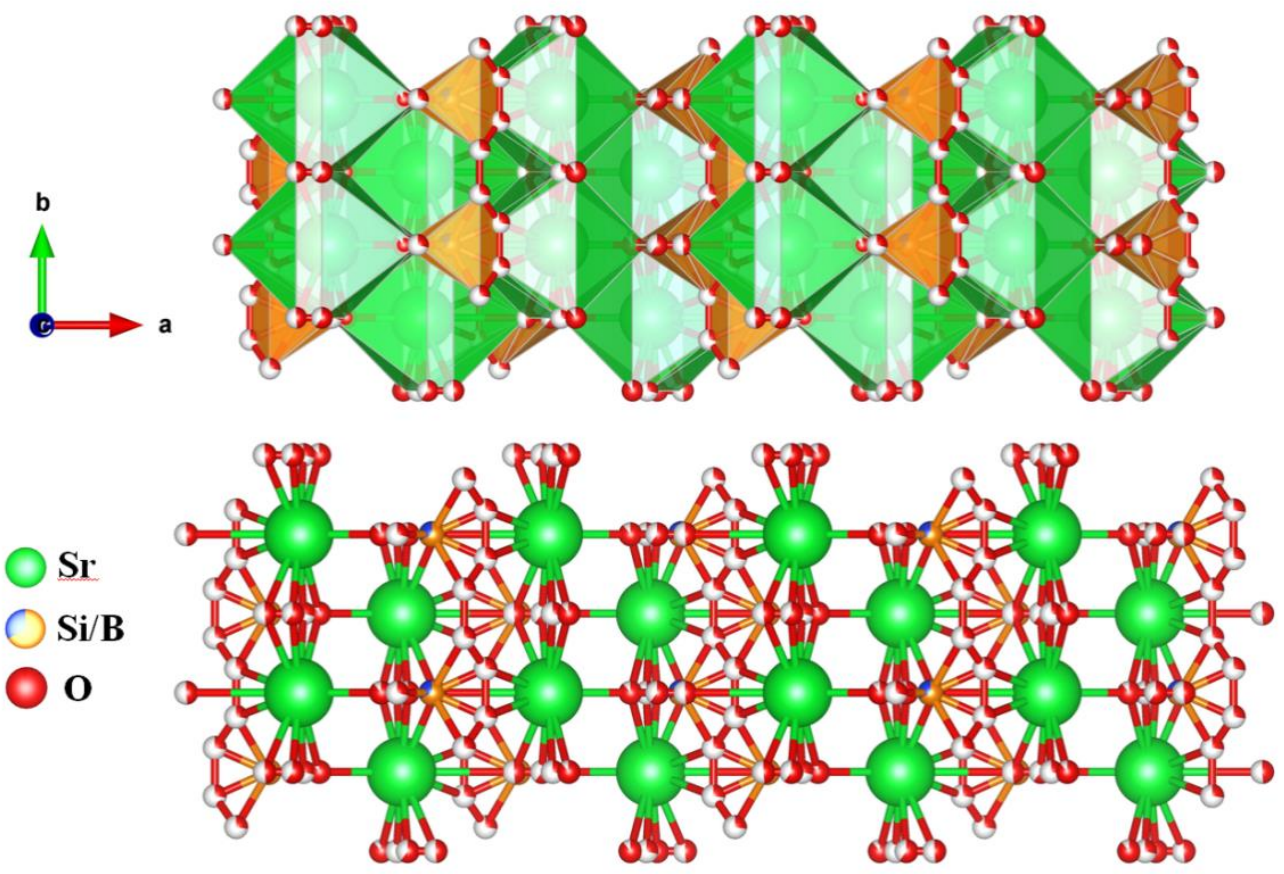

Fig. 2 The crystal structure of $\mathrm{Sr}_{3} \mathrm{~B}_{2} \mathrm{SiO}_{8}$ host 


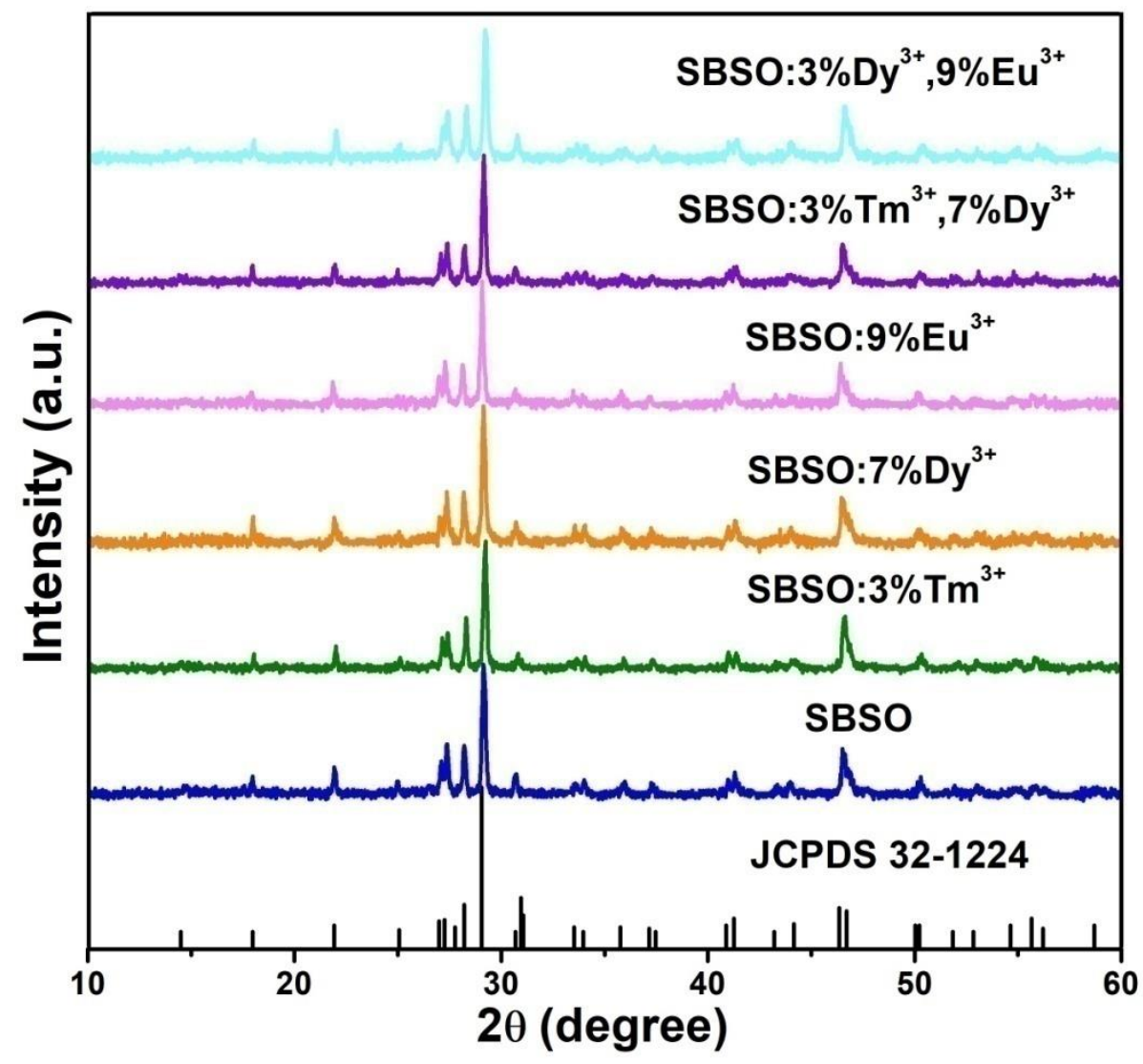

Fig. 3 X-ray powder diffraction patterns of SBSO:Tm ${ }^{3+} / \mathrm{Dy}^{3+} / \mathrm{Eu}^{3+}$ and the standard data (JCPDS 32-1224) for SBSO 


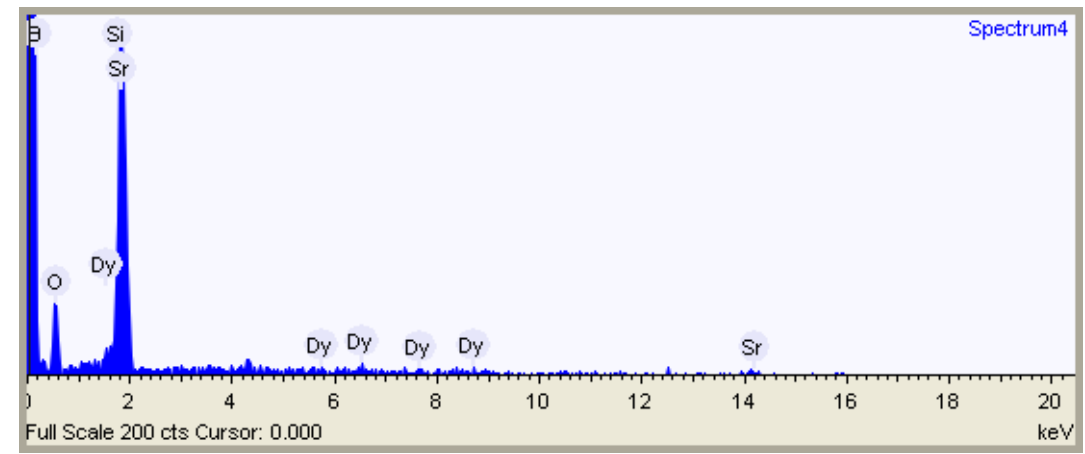

Fig. 4 The EDS spectrum of SBSO:7\% Dy ${ }^{3+}$ sample

Table 3 The EDS quantitative analysis of SBSO:7\% $\mathrm{Dy}^{3+}$ sample

\begin{tabular}{cccc}
\hline Label & Element & Weight $\%$ & Atomic \% \\
\hline 1 & Boron & 5.826 & 15.765 \\
\hline 2 & Oxygen & 32.050 & 58.796 \\
\hline 3 & Silicon & 6.060 & 7.362 \\
\hline 4 & Strontium & 53.092 & 17.722 \\
\hline 5 & Dysprosium & 2.973 & 0.535 \\
\hline
\end{tabular}




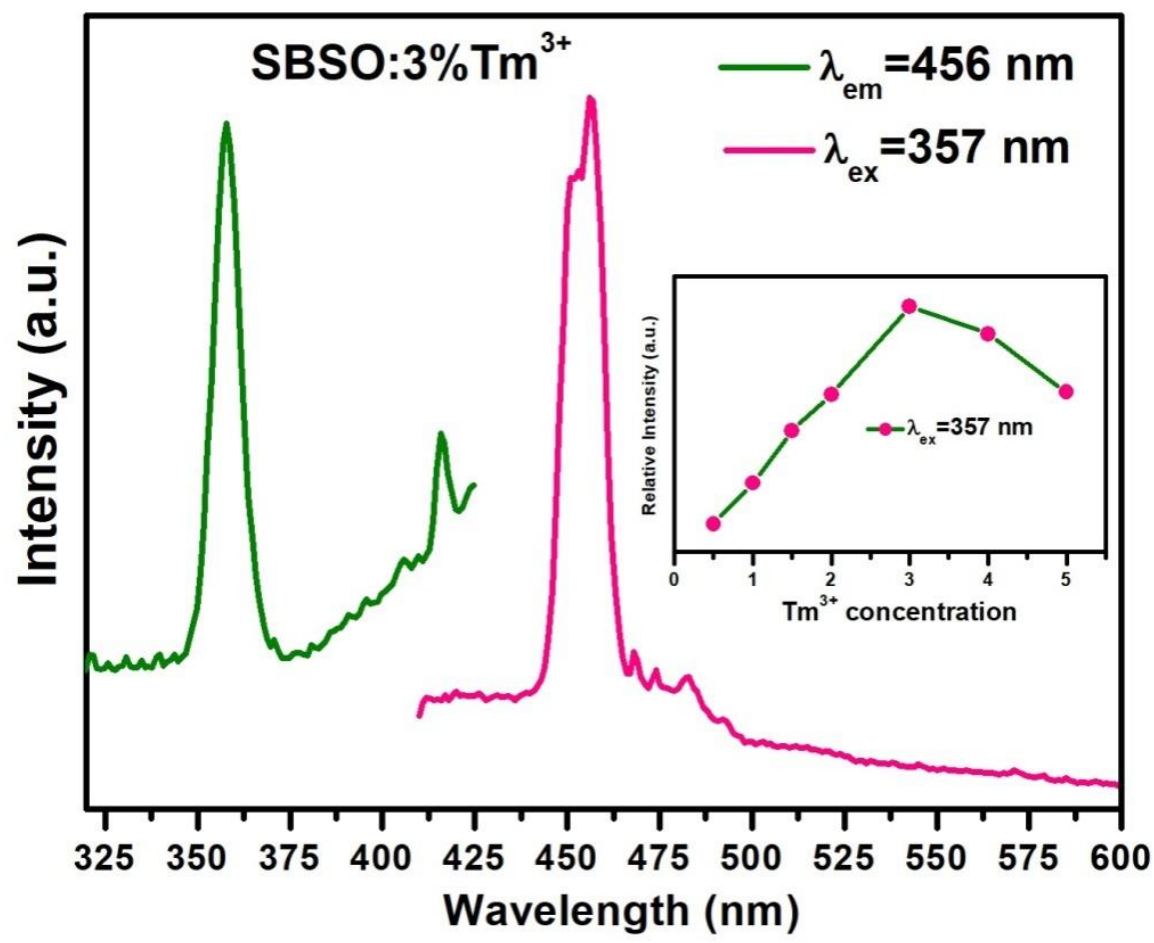

Fig. 5 The excitation and emission spectra of SBSO:3\% $\mathrm{Tm}^{3+}$ phosphor, (the inset) dependence of the emission intensity of SBSO:x\% $\mathrm{Tm}^{3+}$ on $\mathrm{Tm}^{3+}$ concentrations 

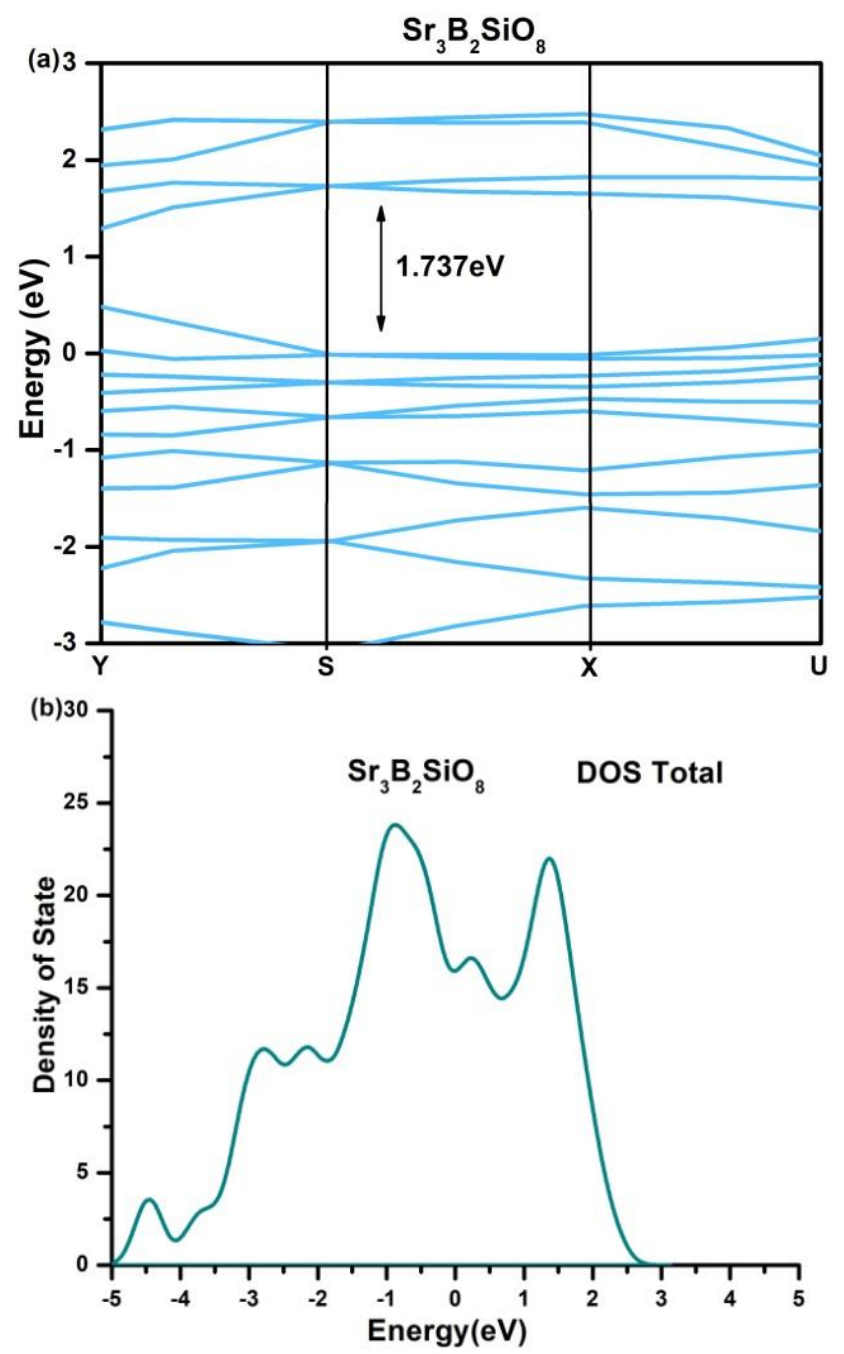

Fig.6 (a) Calculated energy band structure of SBSO (b) Total density of states (DOS) for SBSO calculated by DFT calculation method 

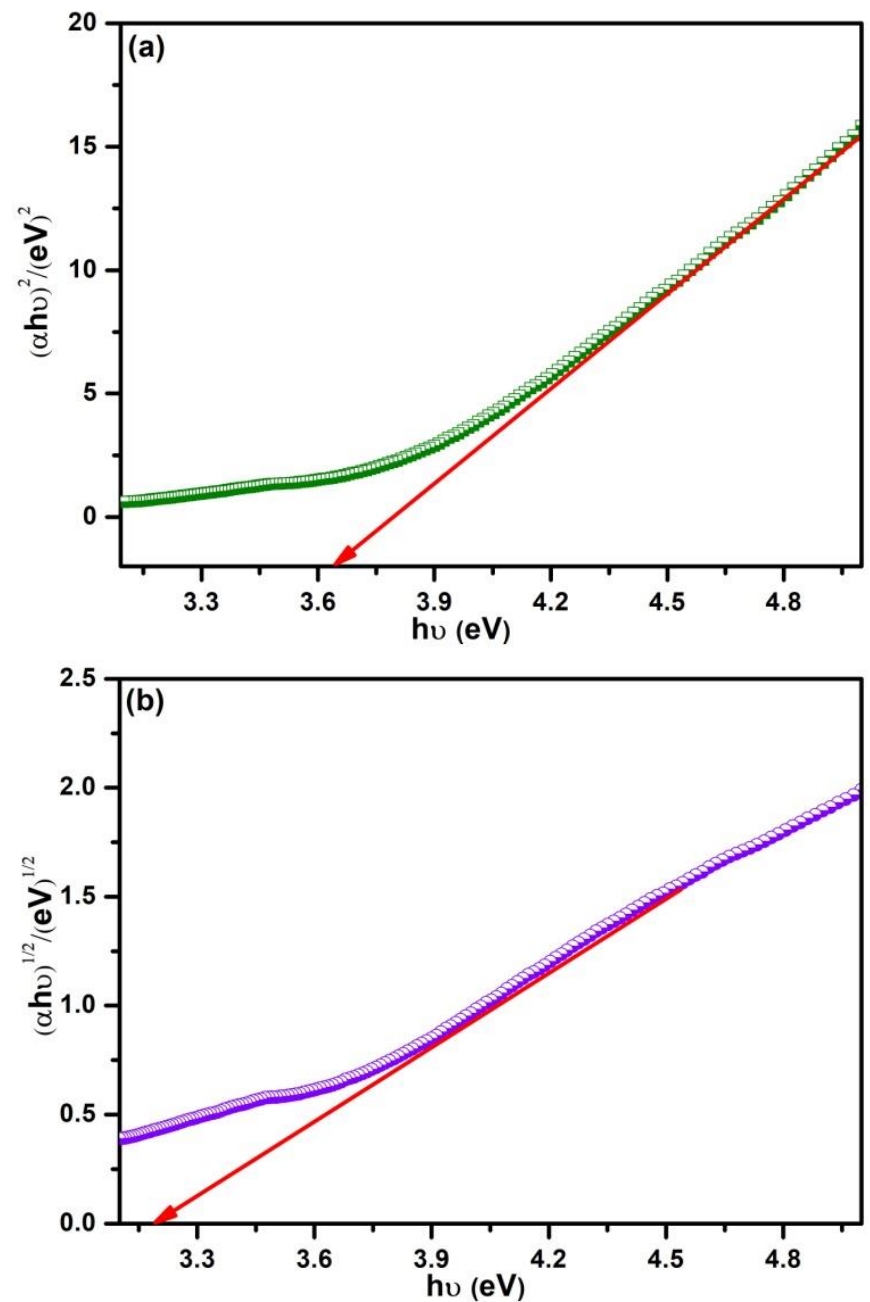

Fig.7 The relationship of $(\alpha \mathrm{hv})^{\mathrm{n}}$ versus photon energy ho to determine the optical band gap of SBSO:3\% $\mathrm{Tm}^{3+}$ for (a) direct and (b) indirect allowed transitions 

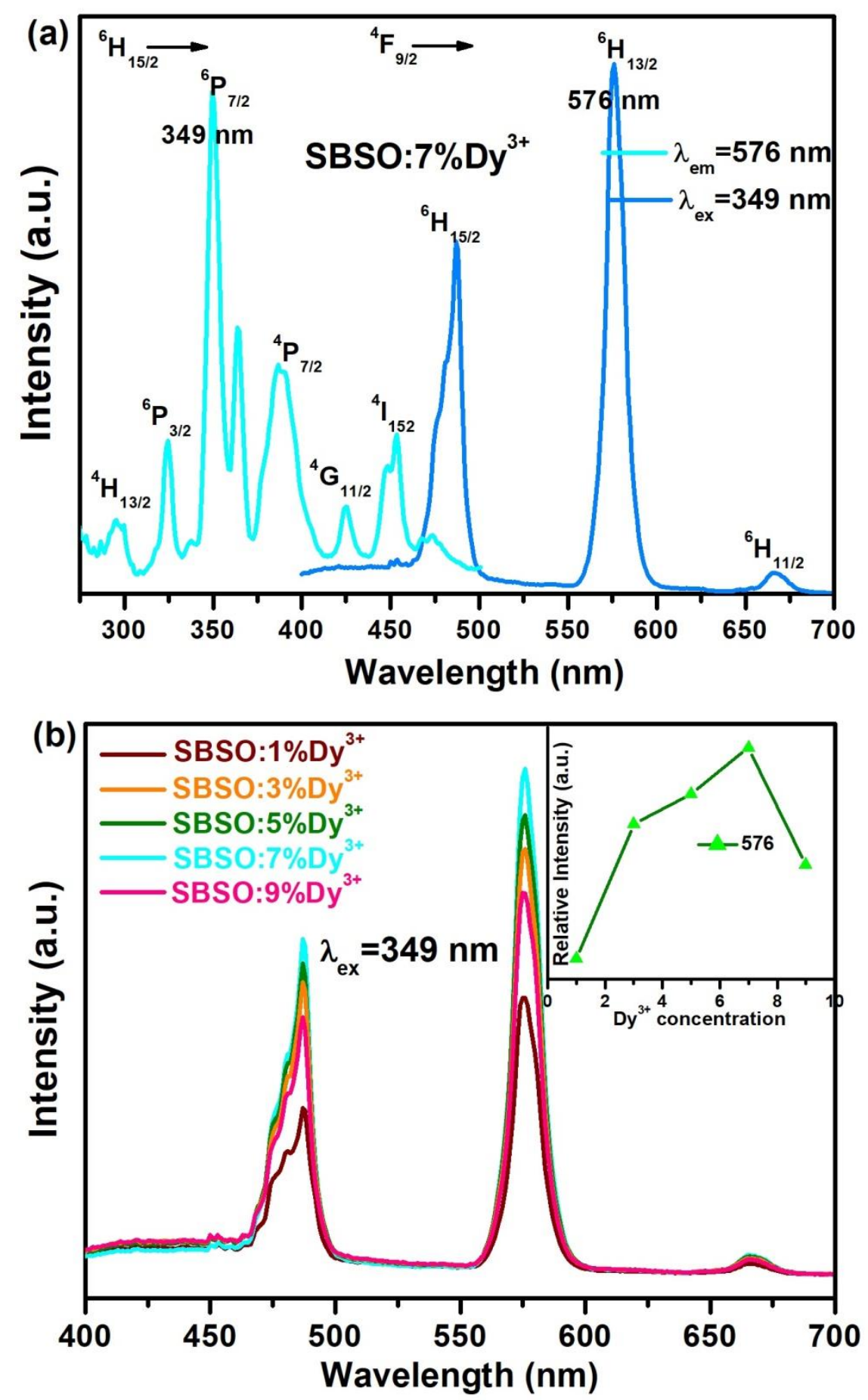

Fig. 8 (a) PL and PLE spectra for SBSO:7\%Dy ${ }^{3+}$ (b) PL spectra for SBSO:x\%Dy ${ }^{3+}(x=1-9)$ with different $\mathrm{Dy}^{3+}$ doped concentrations, the inset shows the optimal concentration curve of $\mathrm{Dy}^{3+}$ 

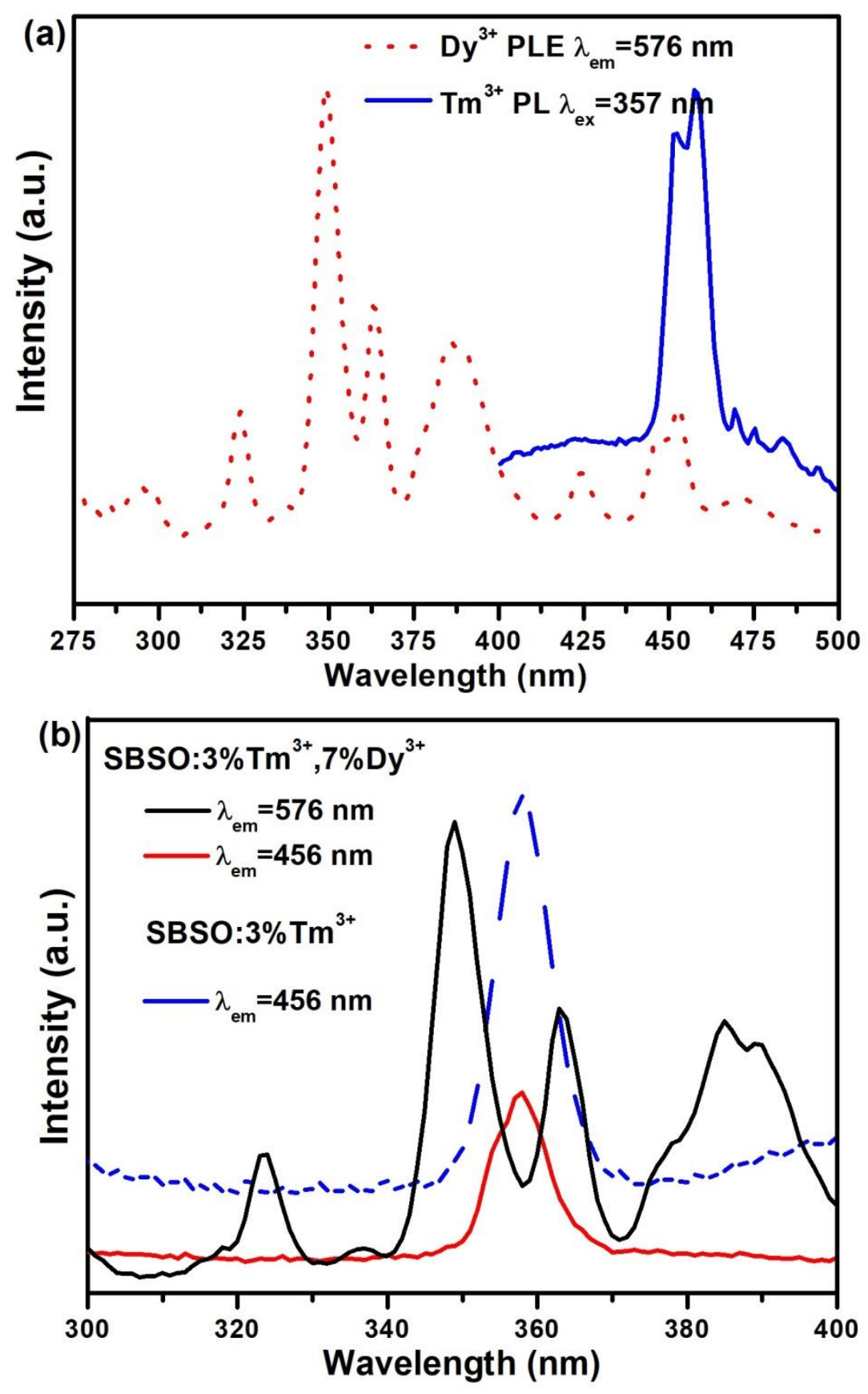

Fig. 9 (a) The corresponding spectral overlapping of $\mathrm{Tm}^{3+}$ PL spectrum for SBSO: $\mathrm{Tm}^{3+}$ and the $\mathrm{Dy}^{3+}$ PLE spectrum for SBSO:Dy ${ }^{3+}(\mathbf{b})$ The PLE spectra of SBSO:Tm ${ }^{3+}, \mathrm{Dy}^{3+}\left(\lambda_{\mathrm{em}}=456\right.$ and 576 $\mathrm{nm})$ and SBSO:Tm${ }^{3+}\left(\lambda_{\mathrm{em}}=456 \mathrm{~nm}\right)$ 

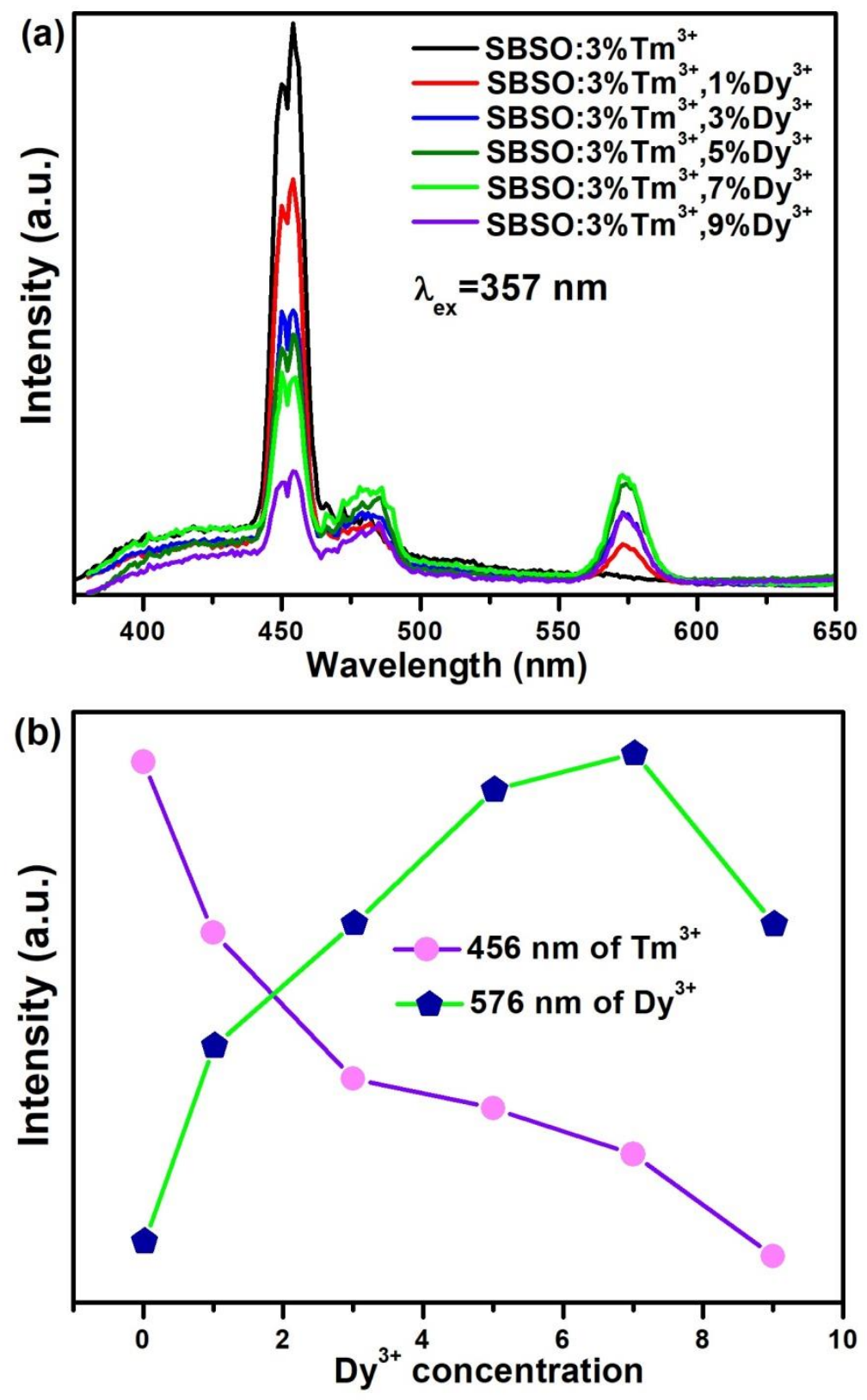

Fig. 10 (a) The emission spectra of SBSO:3\% $\mathrm{Tm}^{3+}, \mathrm{y} \% \mathrm{Dy}^{3+}(\mathrm{y}=0-9)$ with different $\mathrm{Dy}^{3+}$ doped concentrations (b) Dependence of emission intensity on Dy ${ }^{3+}$ concentrations 


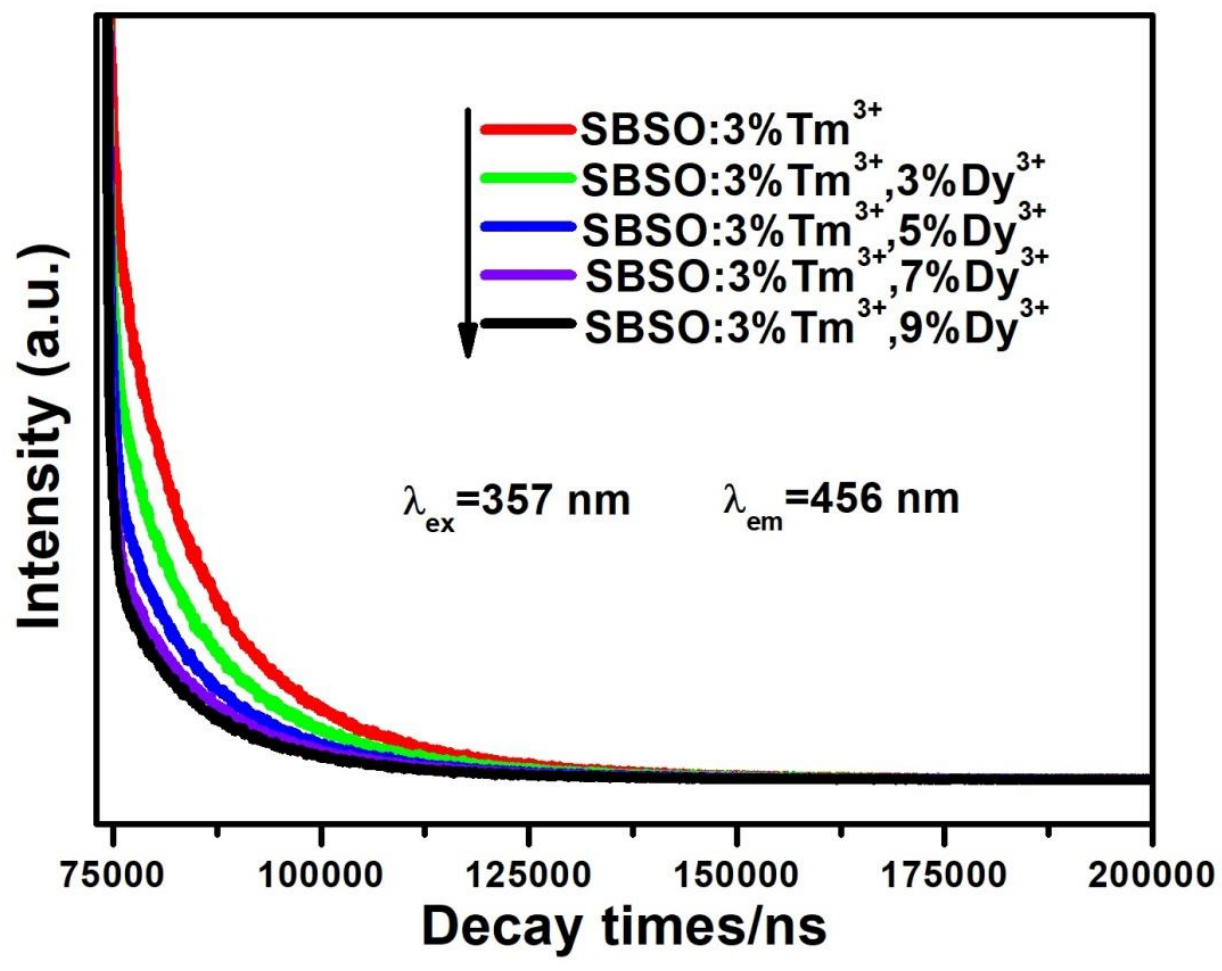

Fig. 11 The decay curves for the luminescence of $\mathrm{Tm}^{3+}$ in SBSO:3\% $\mathrm{Tm}^{3+}, \mathrm{y} \% \mathrm{Dy}^{3+}$ excited at 357 $\mathrm{nm}$, monitored at $456 \mathrm{~nm}$ 


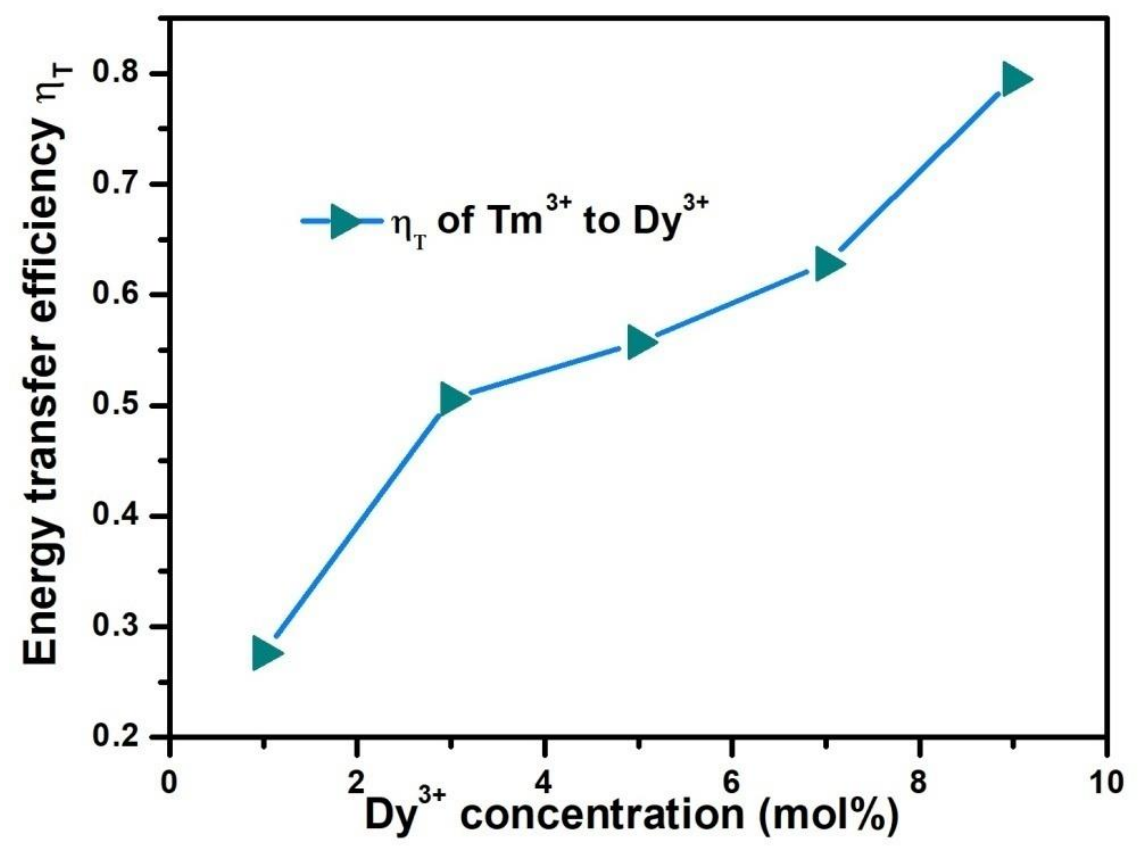

Fig. 12 Dependence of energy transfer efficiency $\eta_{\mathrm{T}}$ on Dy ${ }^{3+}$ concentrations in SBSO:3\% $\mathrm{Tm}^{3+}$, $\mathrm{y} \% \mathrm{Dy}^{3+}$ 


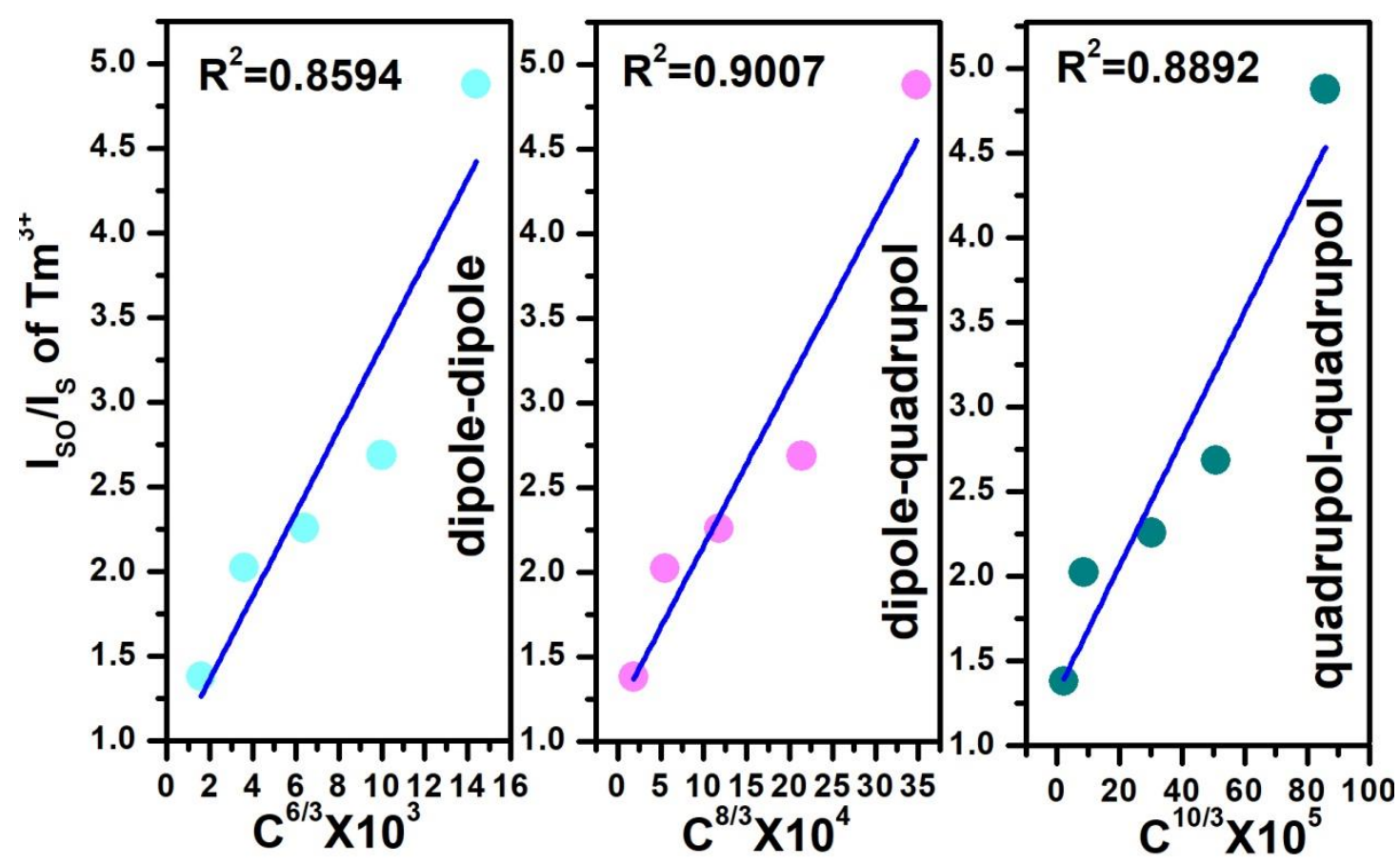

Fig. 13 Dependence of $\mathrm{I}_{\mathrm{so}} / \mathrm{I}_{\mathrm{s}}$ of $\mathrm{Tm}^{3+}$ on $(\mathbf{a}) \mathrm{C}^{6 / 3}$, (b) $\mathrm{C}^{8 / 3}$, and (c) $\mathrm{C}^{10 / 3}$ 

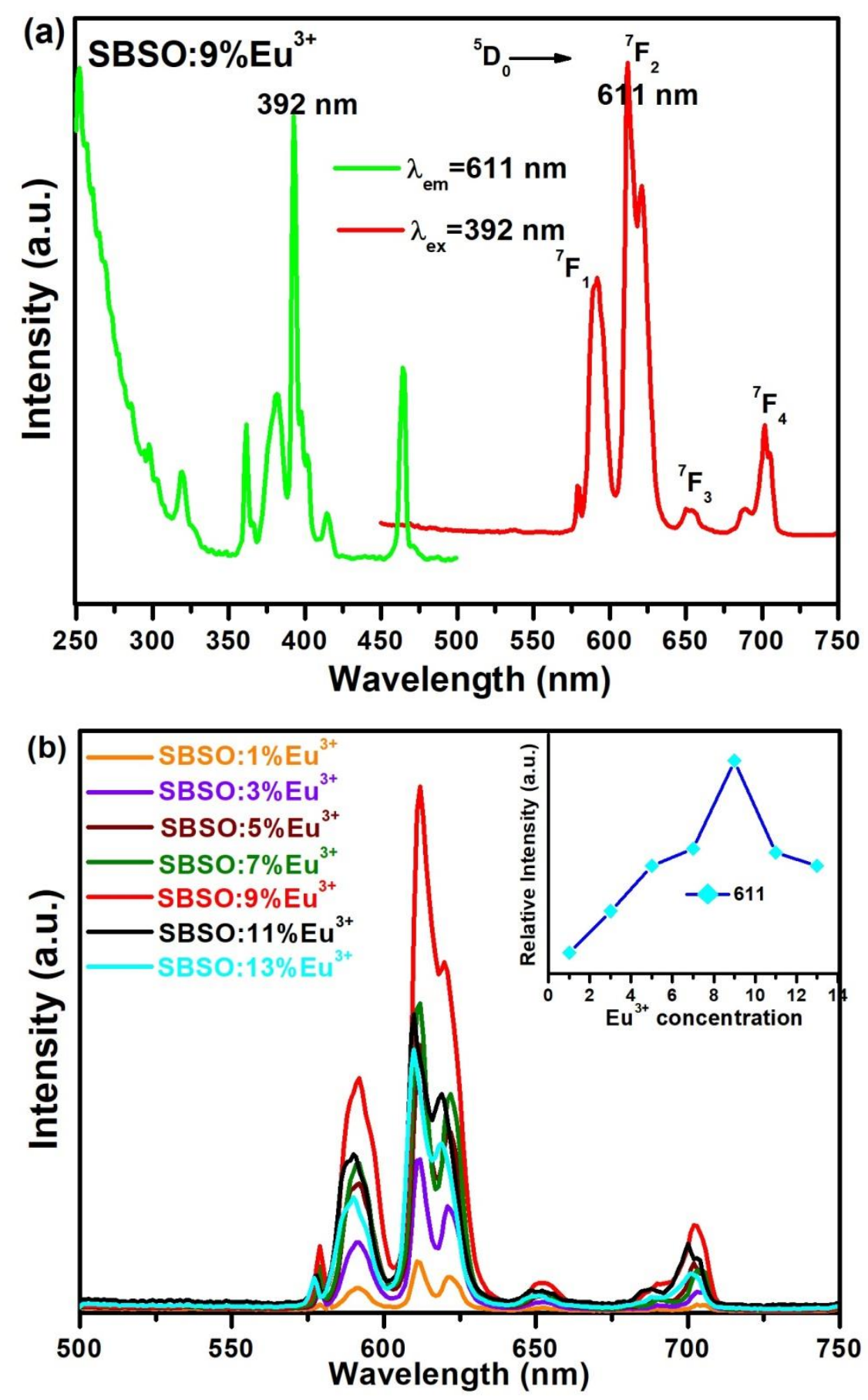

Fig. 14 (a) The excitation and emission spectra of SBSO:9\% $\mathrm{Eu}^{3+}$ (b) The emission spectra of SBSO: $\mathrm{x} \% \mathrm{Eu}^{3+}(\mathrm{x}=1-13)$, the inset shows the relative intensity of $\mathrm{Eu}^{3+}$ ion with different $\mathrm{Eu}^{3+}$ concentrations 


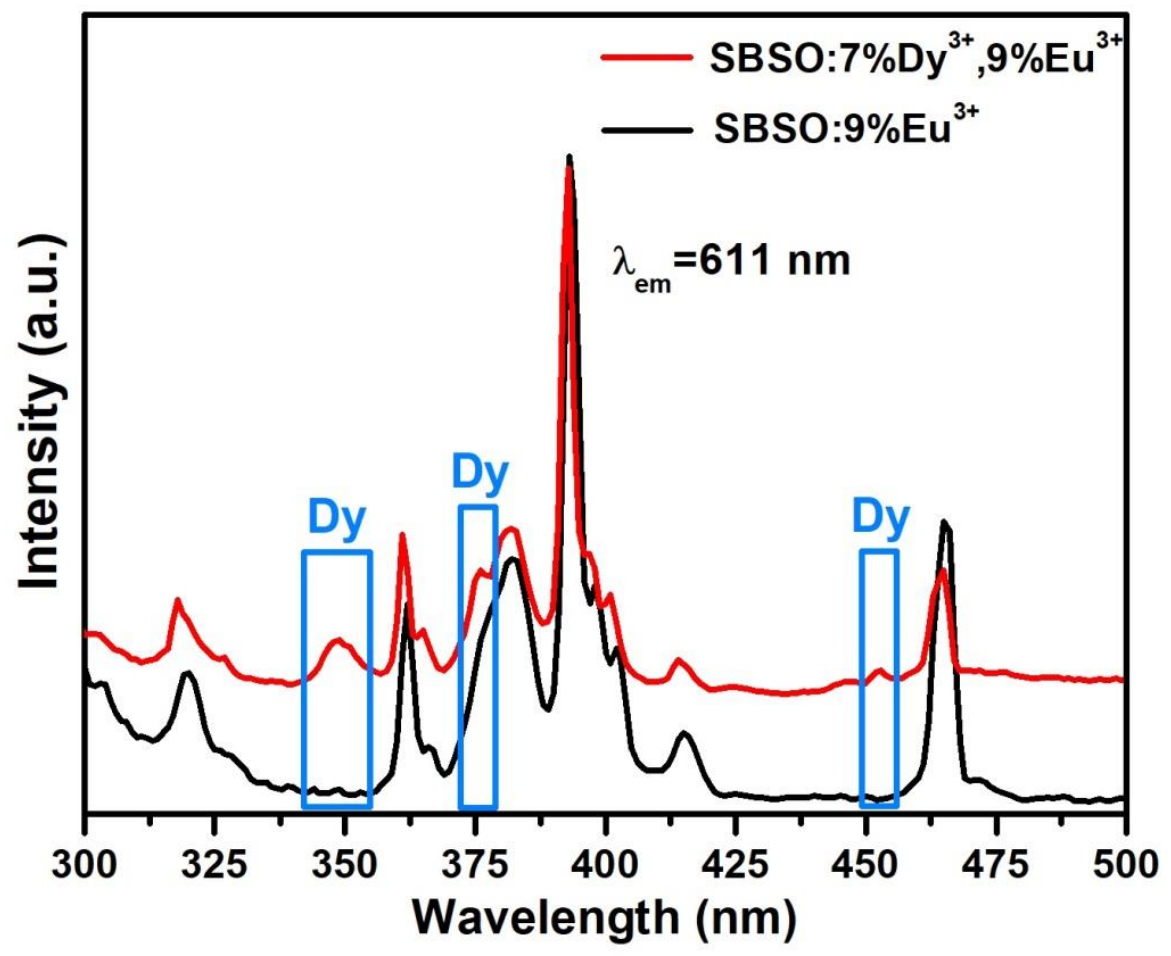

Fig. 15 The PLE spectra for SBSO:9\% $\mathrm{Eu}^{3+}$ and SBSO:7\% $\mathrm{Dy}^{3+}, 9 \% \mathrm{Eu}^{3+}$ both monitored by the emission of $\mathrm{Eu}^{3+}$ at $611 \mathrm{~nm}$ 

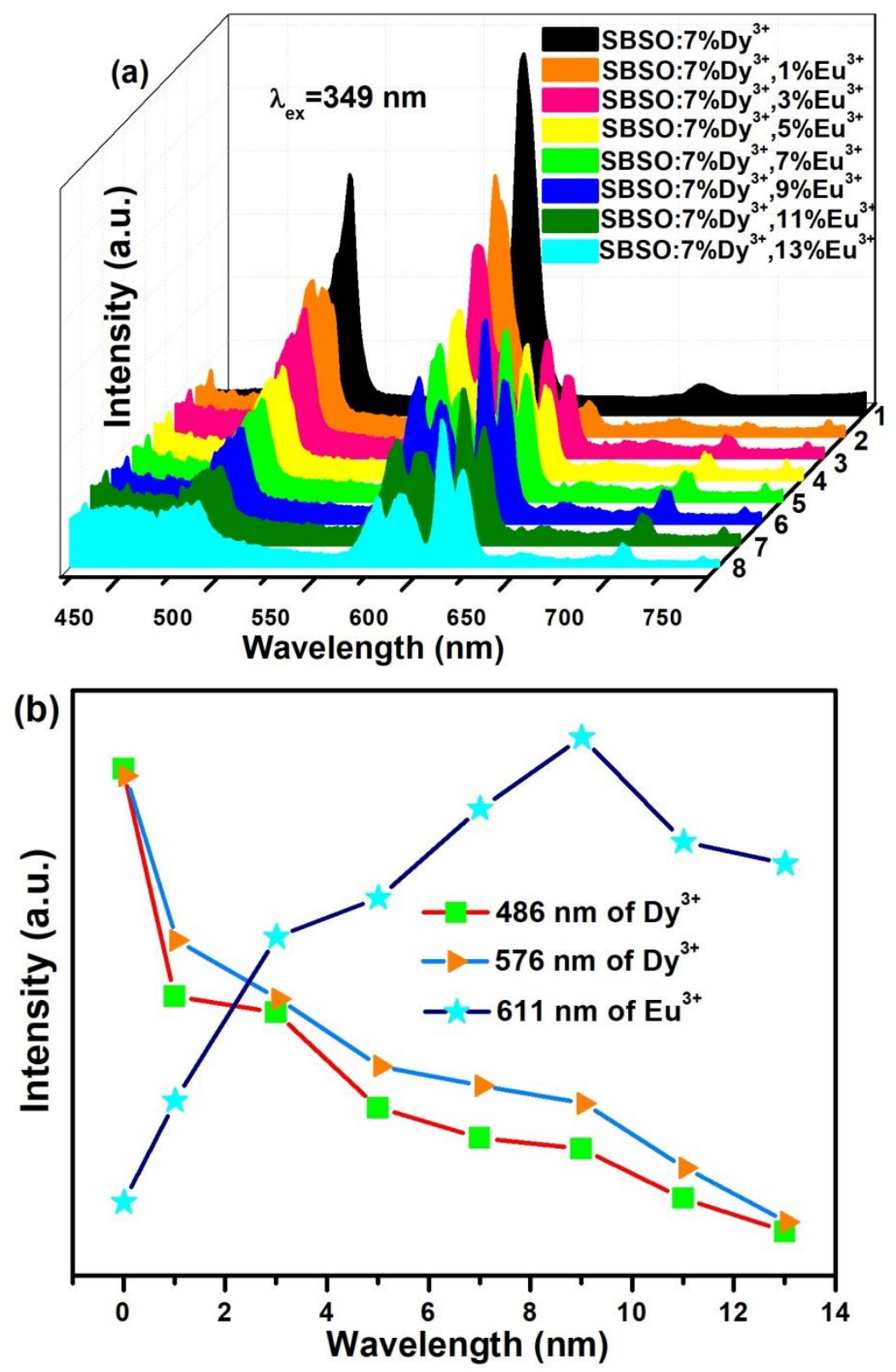

Fig. 16 (a) PL spectra for $\mathrm{SBSO} 7 \% \mathrm{Dy}^{3+}, \mathrm{y}^{2} \mathrm{Eu}^{3+}$ phosphors with different $\mathrm{Eu}^{3+}$ doped concentrations (b) Dependence of emission intensity on $\mathrm{Eu}^{3+}$ concentrations 


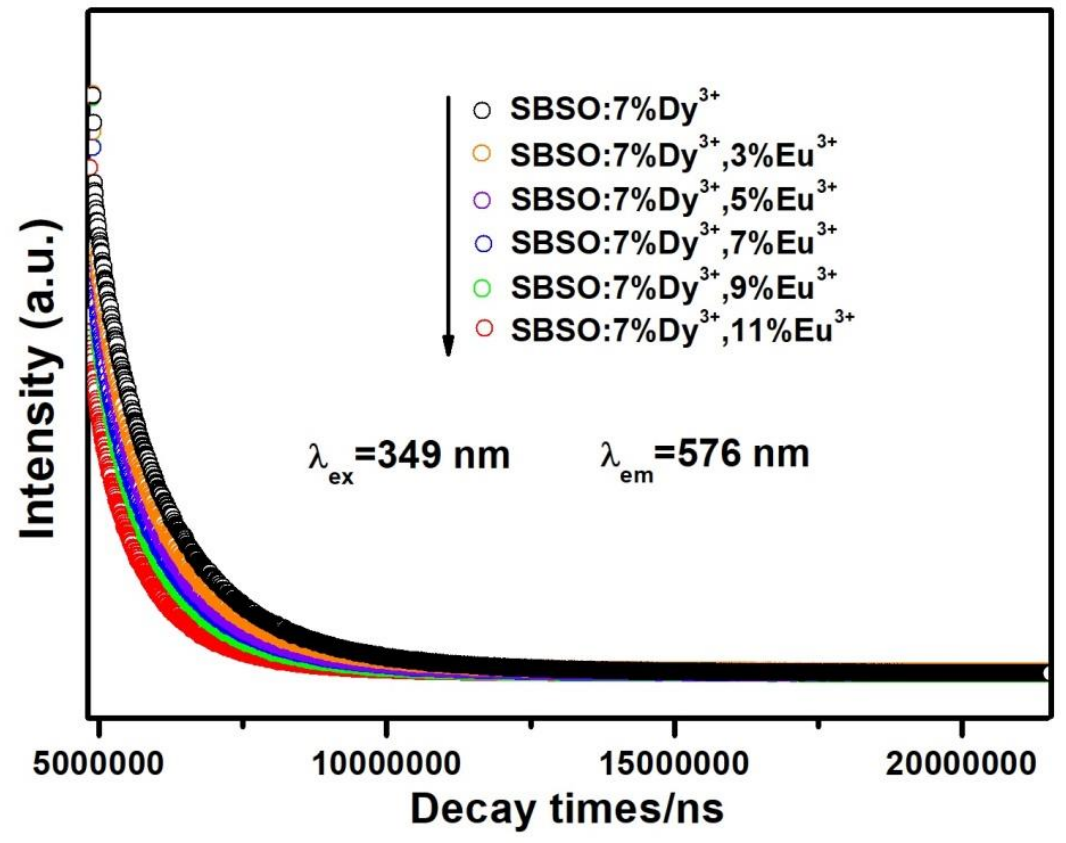

Fig. 17 Decay lifetime tests of SBSO:7\% $\mathrm{Dy}^{3+}, \mathrm{yEu}^{3+}$ detected at $576 \mathrm{~nm}$ for $\mathrm{Eu}^{3+}$ emission excited at $349 \mathrm{~nm}$ 


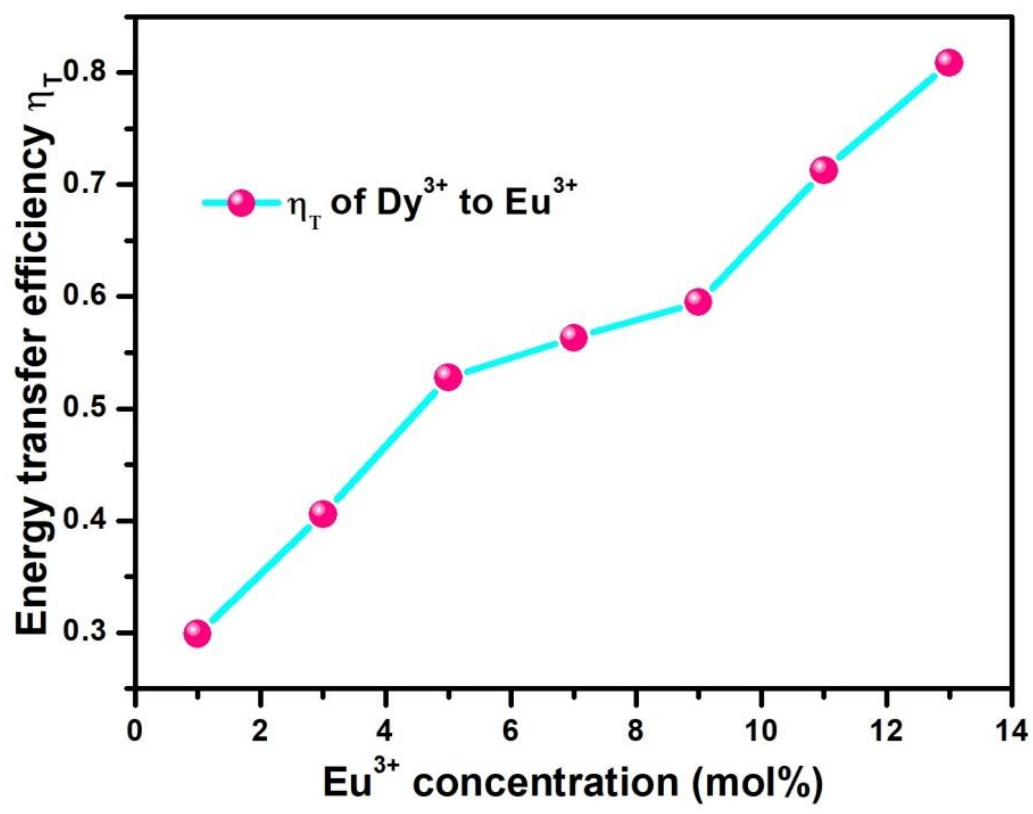

Fig. 18 Energy-transfer efficiency of $\mathrm{Dy}^{3+}-\mathrm{Eu}^{3+}$ on $\mathrm{Eu}^{3+}$ doping concentration for SBSO:7\% $\mathrm{Dy}^{3+}$, $\mathrm{y} \% \mathrm{Eu}^{3+}$ phosphors 


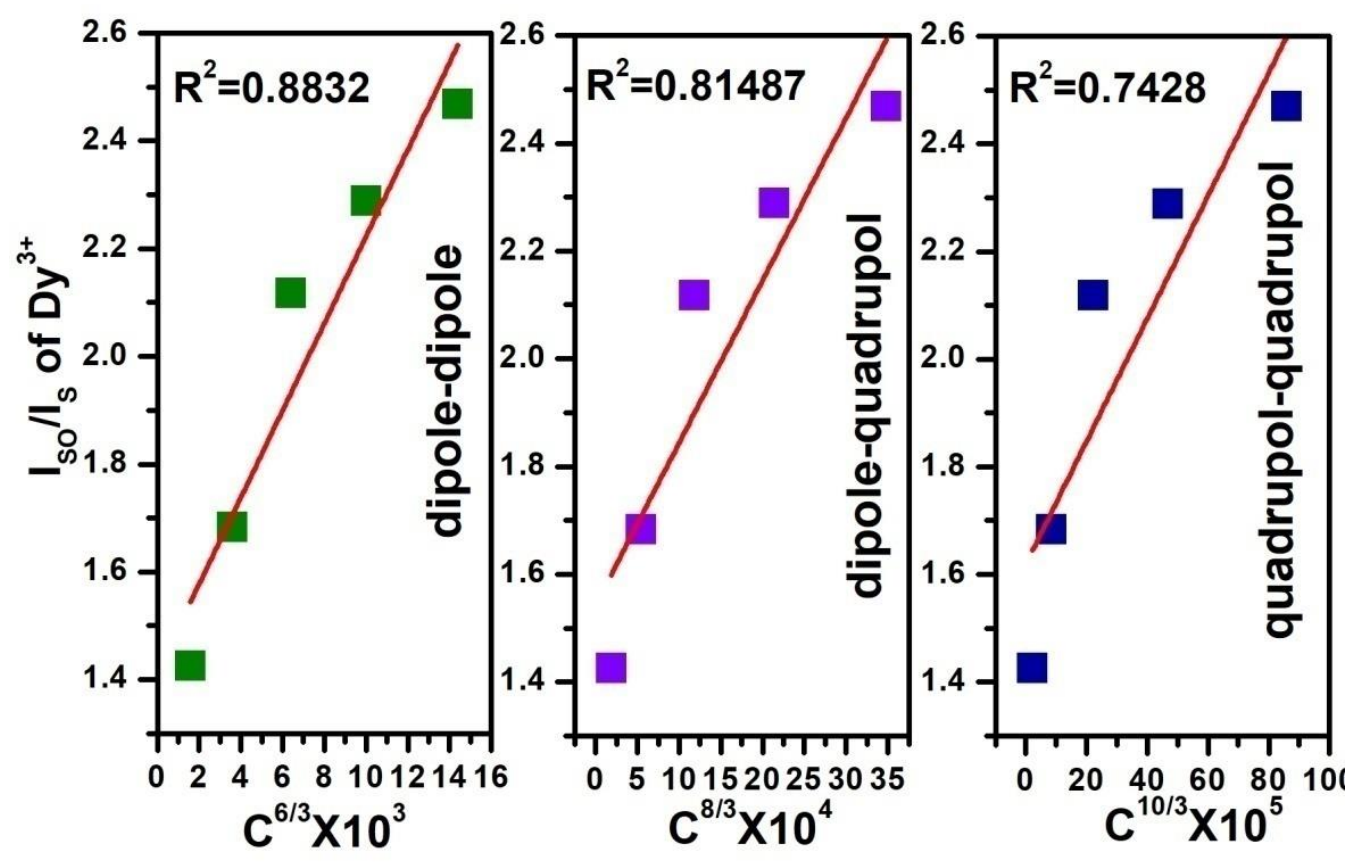

Fig. 19 Dependence of $I_{s o} / I_{s}$ of $D y^{3+}$ on (a) $C^{6 / 3}$, (b) $C^{8 / 3}$, and (c) $C^{10 / 3}$ 

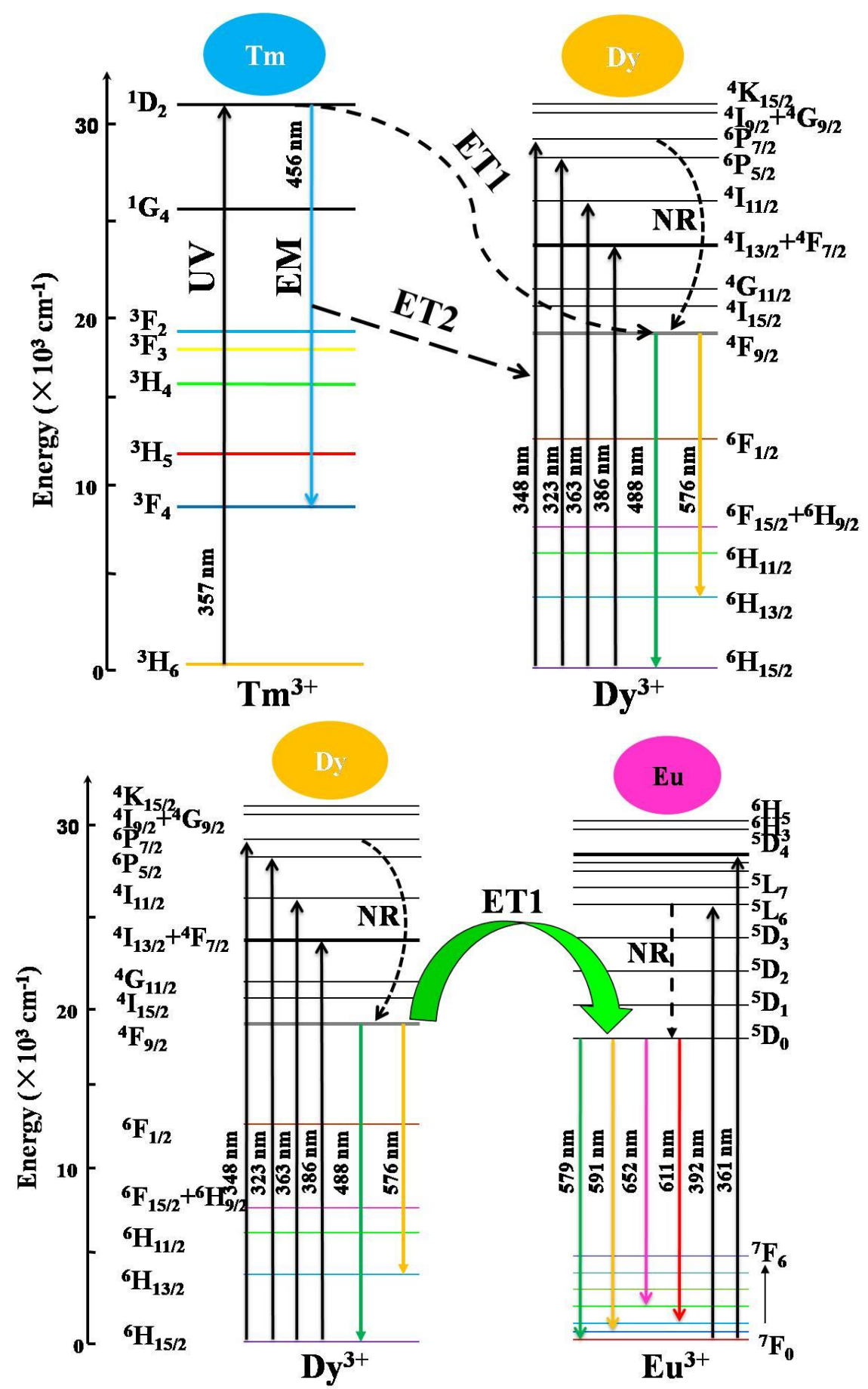

Fig. 20 Schematic energy-level diagram of energy transfer mechanism in the SBSO: $\mathrm{Tm}^{3+} / \mathrm{Dy}^{+} / \mathrm{Eu}^{3+}$ system 


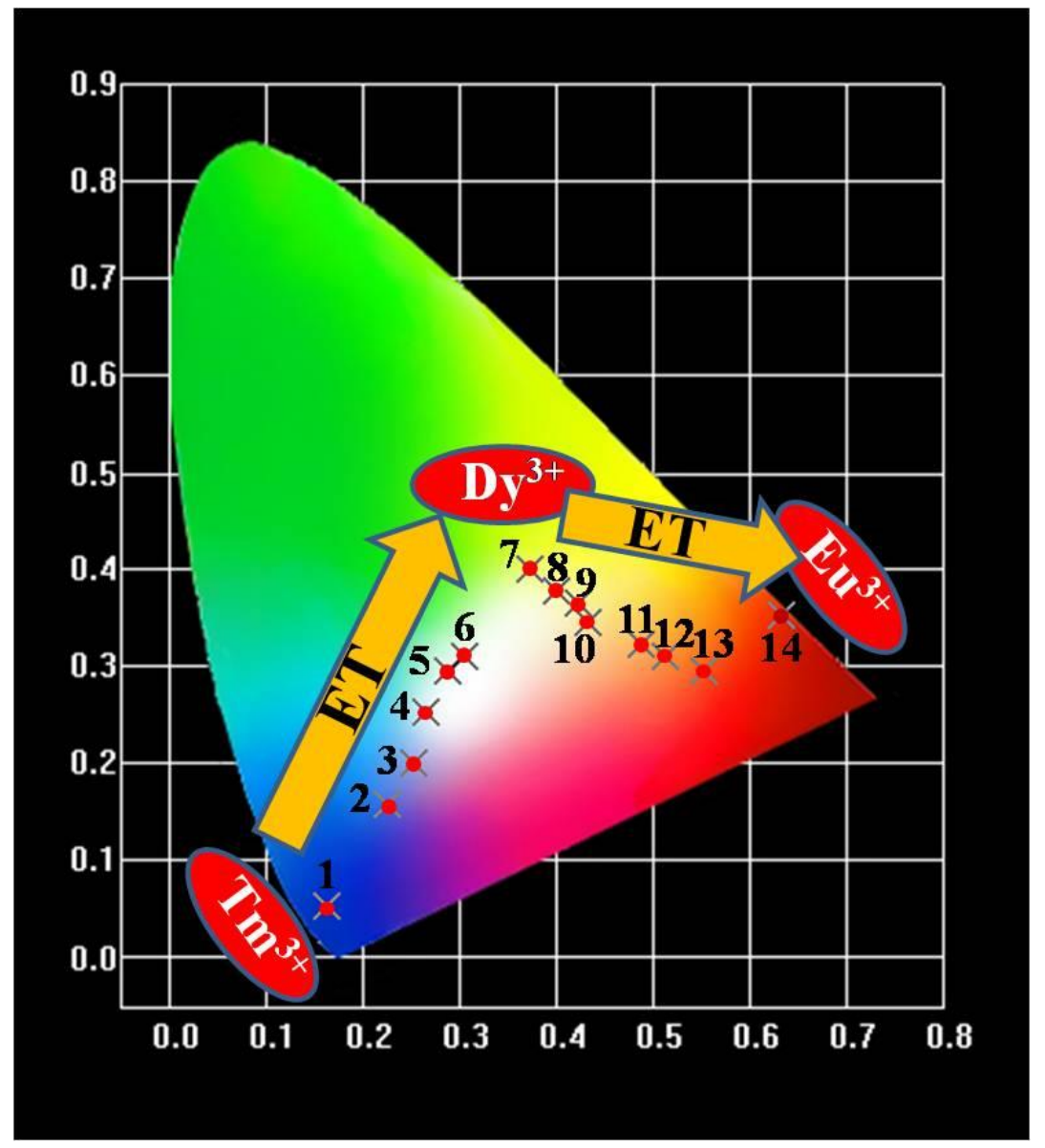

Fig. 21 The CIE chromaticity coordinates for SBSO: $\mathrm{Tm}^{3+} / \mathrm{Dy}^{3+} / \mathrm{Eu}^{3+}$ phosphors under 357 and 349 $\mathrm{nm}$ excitation 
Table 4 The CIE chromaticity coordinates for SBSO: $\mathrm{Tm}^{3+} / \mathrm{Dy}^{3+} / \mathrm{Eu}^{3+}$ phosphors

\begin{tabular}{|c|c|c|c|}
\hline Label & sample & $\operatorname{CIE}(x, y)$ & Color \\
\hline 1 & SBSO: $3 \% \mathrm{Tm}^{3+}$ & $(0.163,0.052)$ & Blue \\
\hline 2 & SBSO: $3 \% \mathrm{Tm}^{3+}, 1 \% \mathrm{Dy}^{3+}$ & $(0.226,0.158)$ & Blue \\
\hline 3 & SBSO: $3 \% \mathrm{Tm}^{3+}, 3 \% \mathrm{Dy}^{3+}$ & $(0.253,0.200)$ & Blue \\
\hline 4 & SBSO: $3 \% \mathrm{Tm}^{3+}, 5 \% \mathrm{Dy}^{3+}$ & $(0.264,0.253)$ & White \\
\hline 5 & SBSO: $3 \% \mathrm{Tm}^{3+}, 7 \% \mathrm{Dy}^{3+}$ & $(0.287,0.294)$ & White \\
\hline 6 & SBSO: $3 \% \mathrm{Tm}^{3+}, 9 \% \mathrm{Dy}^{3+}$ & $(0.305,0.310)$ & White \\
\hline 7 & SBSO:7\% $\mathrm{Dy}^{3+}$ & $(0.372,0.401)$ & Yellow \\
\hline 8 & SBSO: $7 \% \mathrm{Dy}^{3+}, 1 \% \mathrm{Eu}^{3+}$ & $(0.401,0.378)$ & Yellow \\
\hline 9 & SBSO:7\%Dy ${ }^{3+}, 3 \% \mathrm{Eu}^{3+}$ & $(0.423,0.365)$ & Yellow \\
\hline 10 & SBSO: $7 \% \mathrm{Dy}^{3+}, 5 \% \mathrm{Eu}^{3+}$ & $(0.432,0.344)$ & Yellow \\
\hline 11 & SBSO:7\% $\mathrm{Dy}^{3+}, 7 \% \mathrm{Eu}^{3+}$ & $(0.487,0.323)$ & Orange-red \\
\hline 12 & SBSO:7\% $\mathrm{Dy}^{3+}, 9 \% \mathrm{Eu}^{3+}$ & $(0.512,0.311)$ & Orange-red \\
\hline 13 & SBSO:7\% $\mathrm{Dy}^{3+}, 13 \% \mathrm{Eu}^{3+}$ & $(0.553,0.395)$ & Orange-red \\
\hline 14 & SBSO:9\% $\mathrm{Eu}^{3+}$ & $(0.633,0.352)$ & Red \\
\hline
\end{tabular}



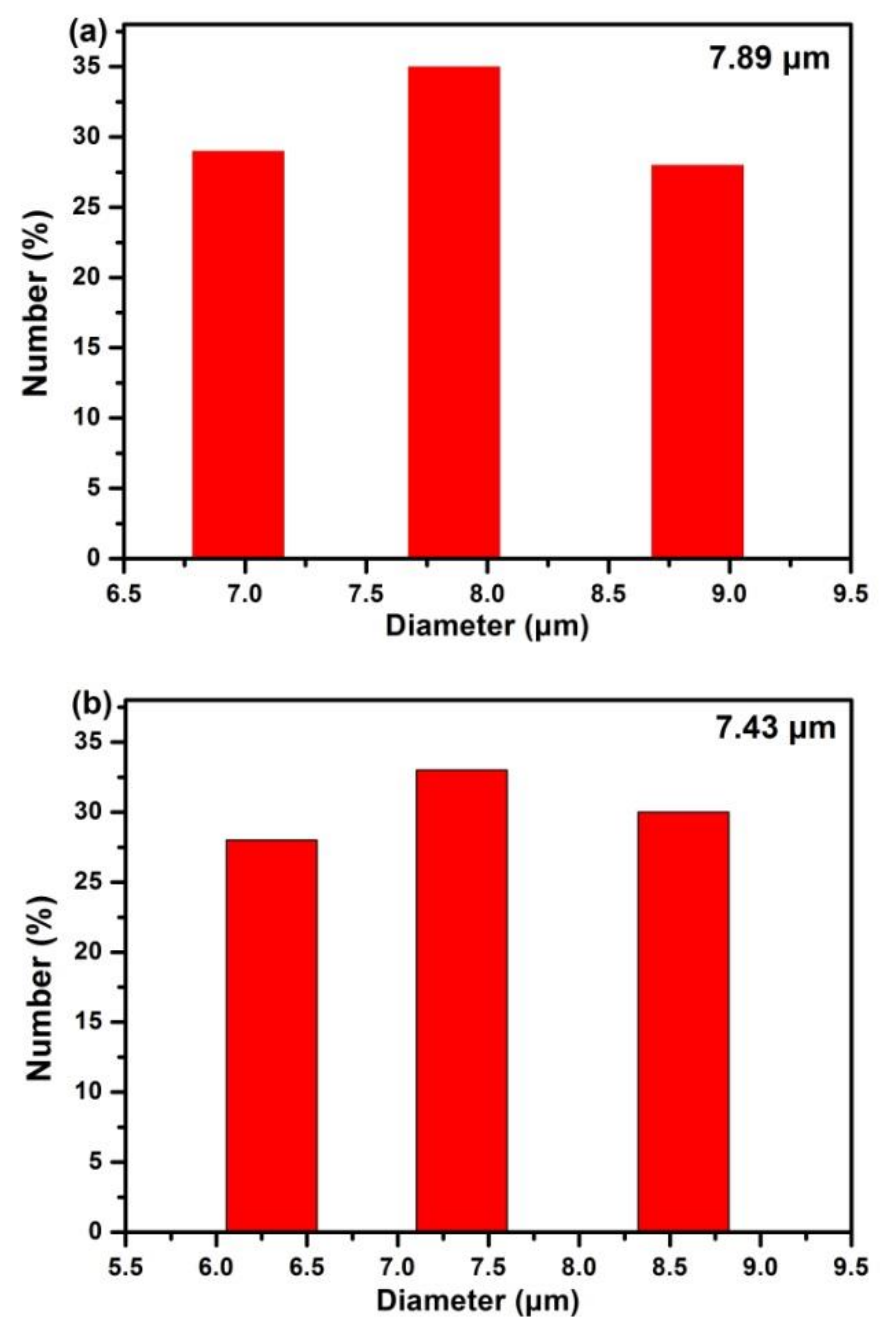

Fig. 22 The particle size distribution of (a) SBSO:3\% $\mathrm{Tm}^{3+}, 7 \% \mathrm{Dy}^{3+}$ and (b) $\mathrm{SBSO}: 7 \% \mathrm{Dy}^{3+}$, $9 \% \mathrm{Eu}^{3+}$ phosphors, the average diameters are 7.89 and $7.43 \mu \mathrm{m}$, respectively 


\section{Declaration}

\section{Funding}

This funding for the current research is received from the Major Program for the Field (No: 2016009) and PhD Start-Up Funding (No: 2017001), Jilin Institute of Chemical Technology.

\section{Conflicts of interest/Competing interests}

The authors declare that we have no known competing financial interests or personal relationships that could have appeared to influence the work reported in the manuscript entitled "Luminescence and tunable color properties of uniphase white-emitting $\mathrm{Sr}_{3} \mathrm{~B}_{2} \mathrm{SiO}_{8}: \mathrm{Tm}^{3+} / \mathrm{Dy}^{3+} / \mathrm{Eu}^{3+}$ phosphors by energy transfer for UV-excited white LEDs".

\section{Availability of data and material}

The authors declare that all data and materials as well as software application or custom code support our published claims and comply with field standards.

\section{Code availability}

The authors declare that all data and materials as well as software application or custom code support our published claims and comply with field standards.

\section{Authors' contributions}

All authors contributed to the study conception and design. Material preparation, data collection and analysis were performed by Bo Yuan, Chaochao Qi and Xiangting Zhang. The first draft of the manuscript was written by Bo Yuan and all authors commented on previous versions of the manuscript. All authors read and approved the final manuscript.

\section{Ethics approval}

Not applicable

\section{Consent to participate}

We confirm that the manuscript has been read and approved by all named authors and that there are no other persons who satisfied the criteria for authorship but are not listed. We further confirm that the order of authors listed in the manuscript has been approved by all of us. 


\section{Consent for publication}

We confirm that we have given due consideration to the protection of intellectual property associated with this work and that there are no impediments to publication, including the timing of publication, with respect to intellectual property. In so doing we confirm that we have followed the regulations of our institutions concerning intellectual property.

Authors: Bo Yuan, Chaochao Qi, Xiangting Zhang, Yanhua Song, Guoyan Luan, Haifeng Zou

Date appended: 2021.02.12 
Figures

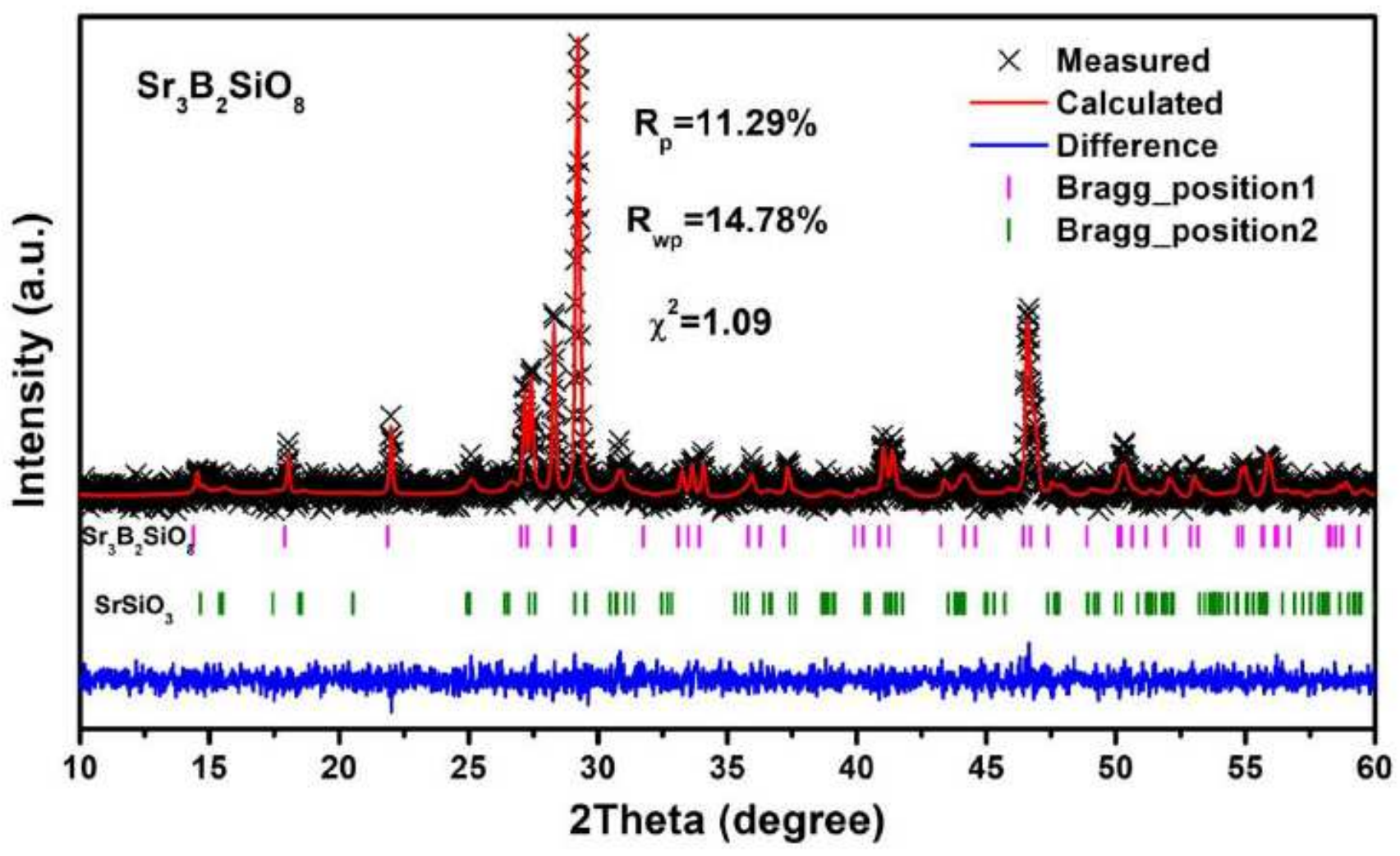

Figure 1

XRD pattern for the Rietveld refinement of Sr3B2SiO8 

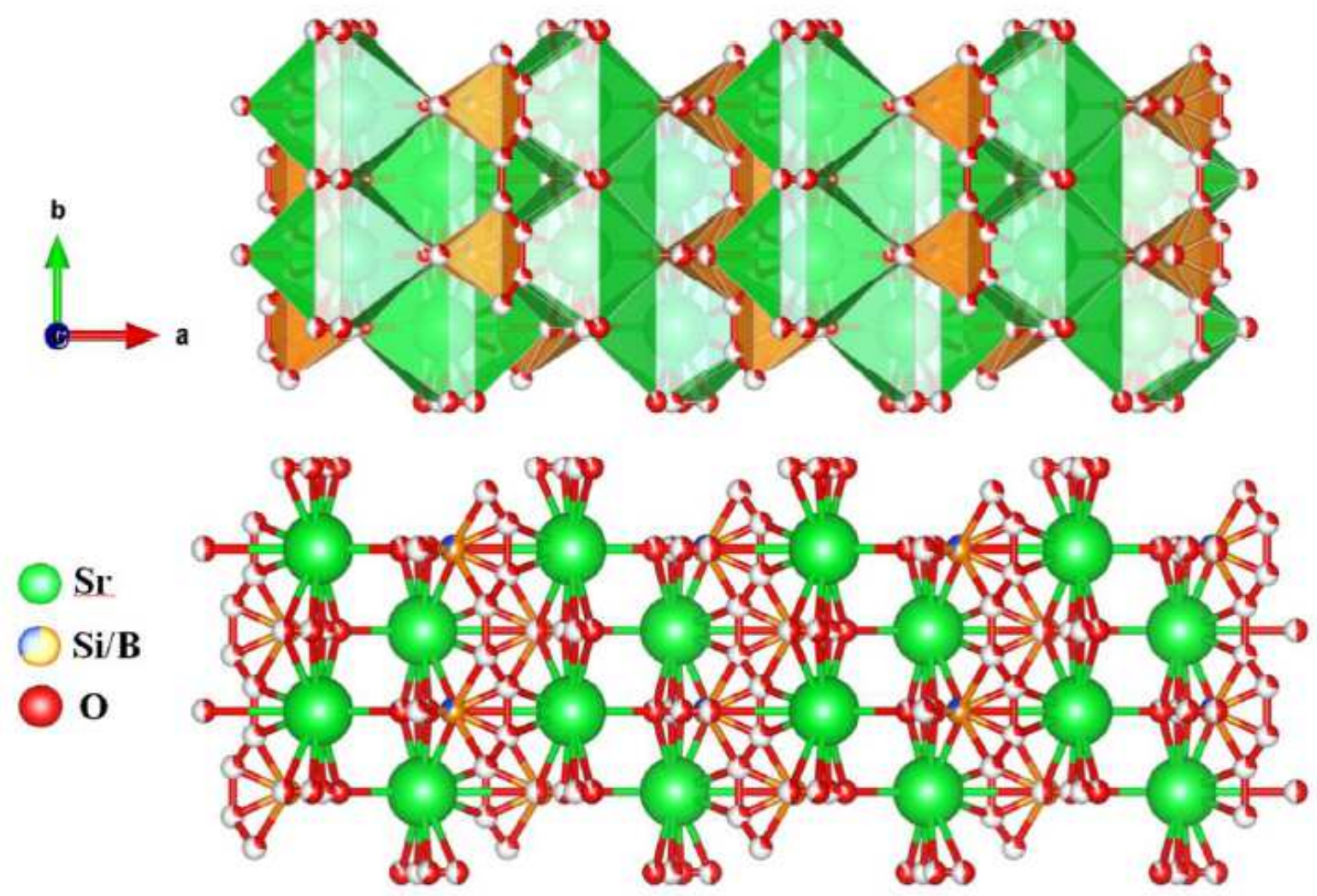

Figure 2

The crystal structure of Sr3B2SiO8 host 


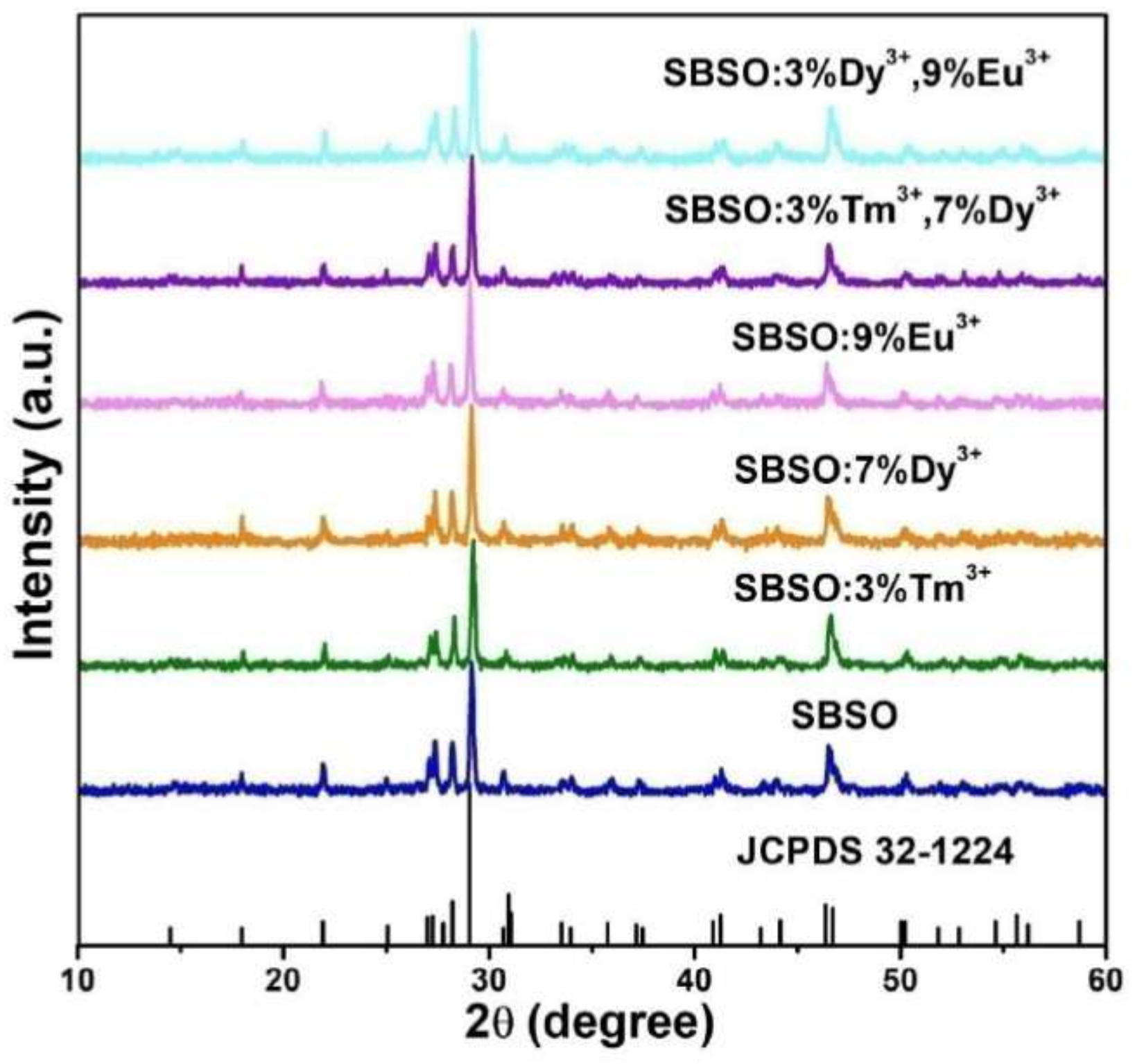

Figure 3

X-ray powder diffraction patterns of SBSO:Tm3+/Dy3+/Eu3+ and the standard data (JCPDS 32-1224) for SBSO 


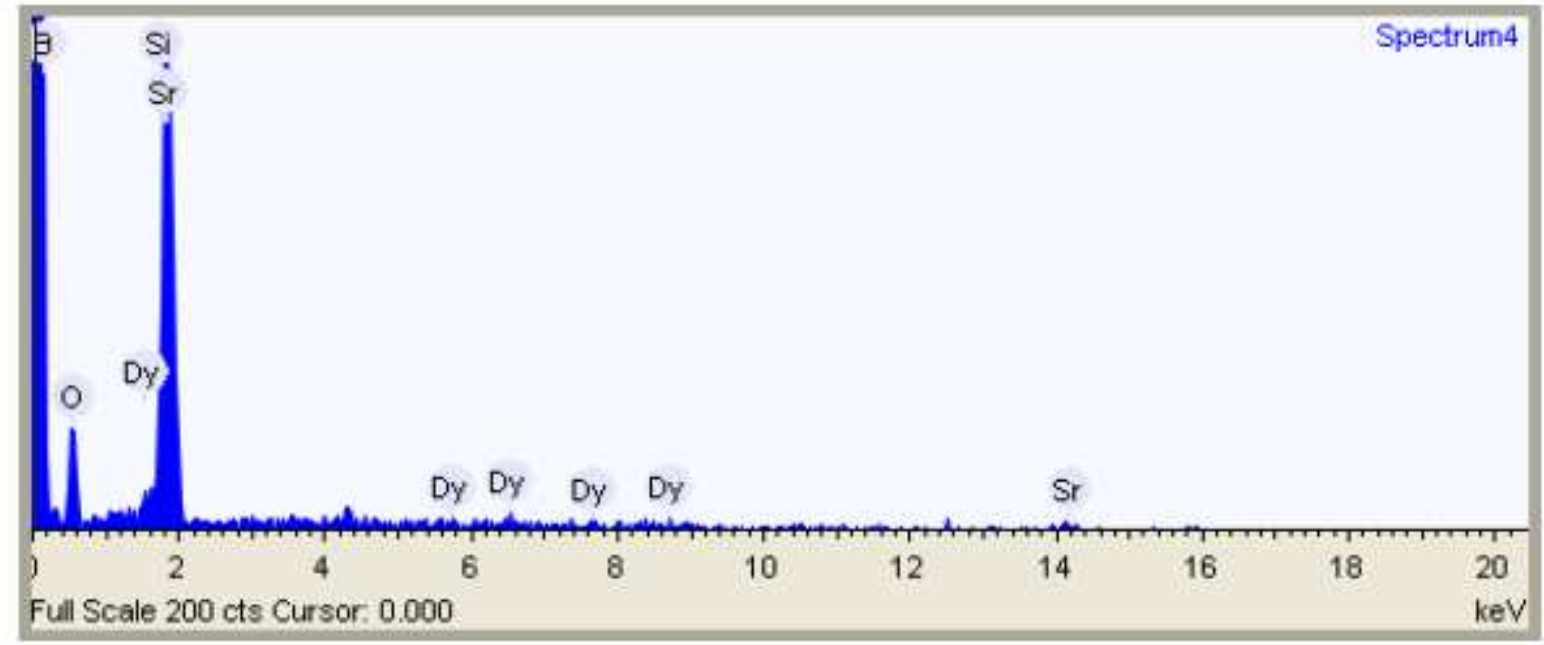

Figure 4

The EDS spectrum of SBSO:7\%Dy3+ sample

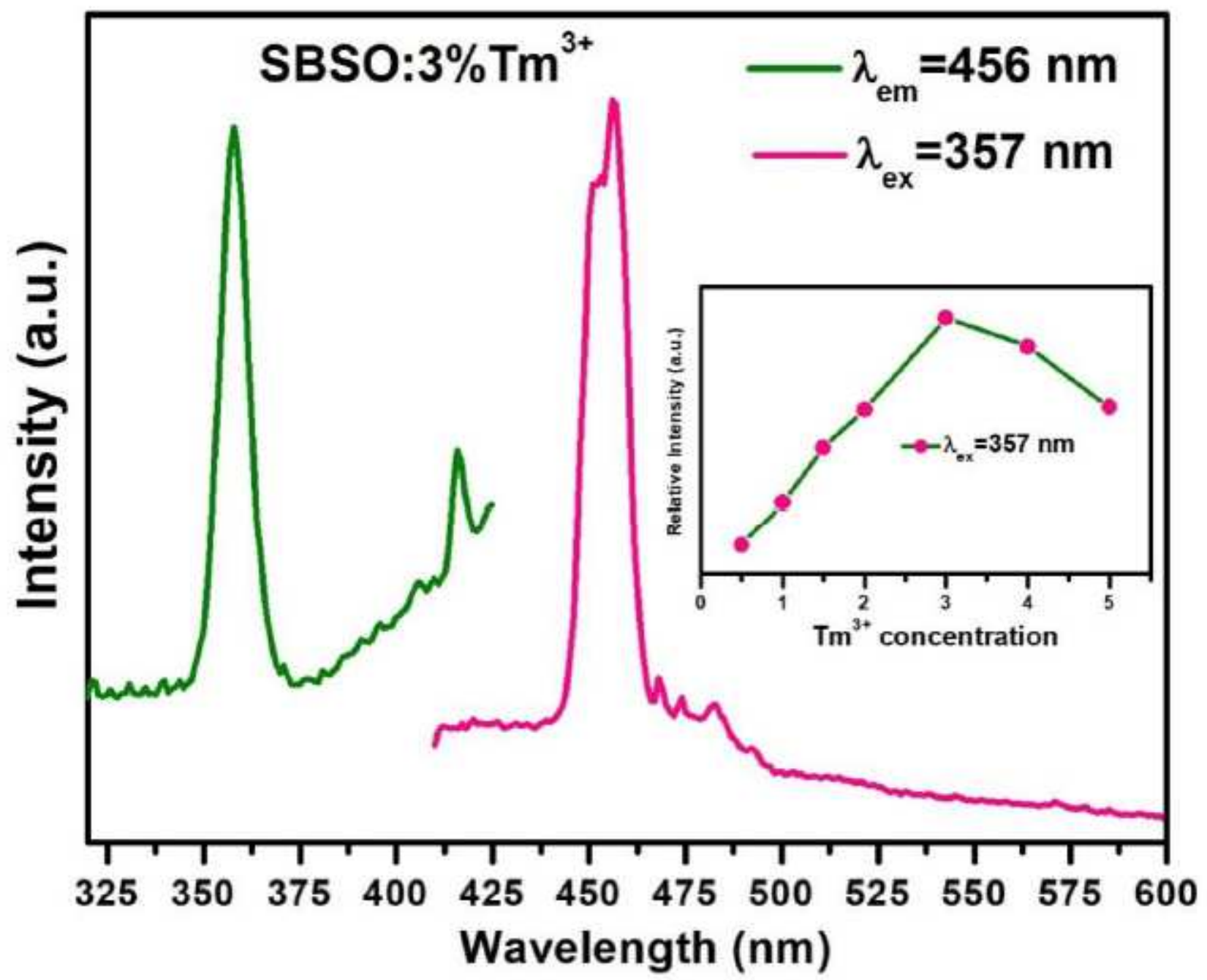


Figure 5

The excitation and emission spectra of SBSO:3\%Tm3+ phosphor, (the inset) dependence of the emission intensity of SBSO:x\%Tm3+ on Tm3+ concentrations
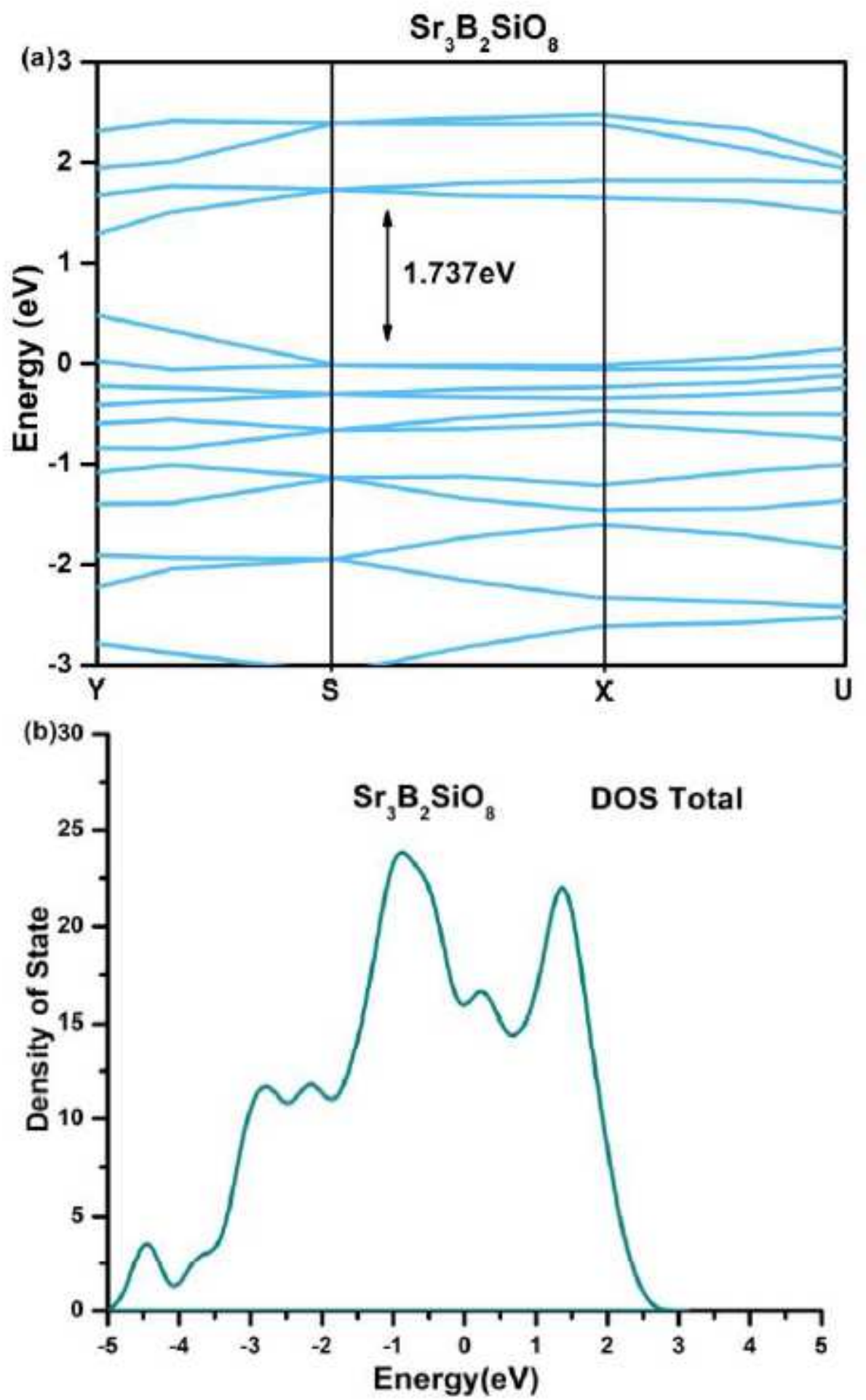

Figure 6 
(a) Calculated energy band structure of SBSO (b) Total density of states (DOS) for SBSO calculated by DFT calculation method
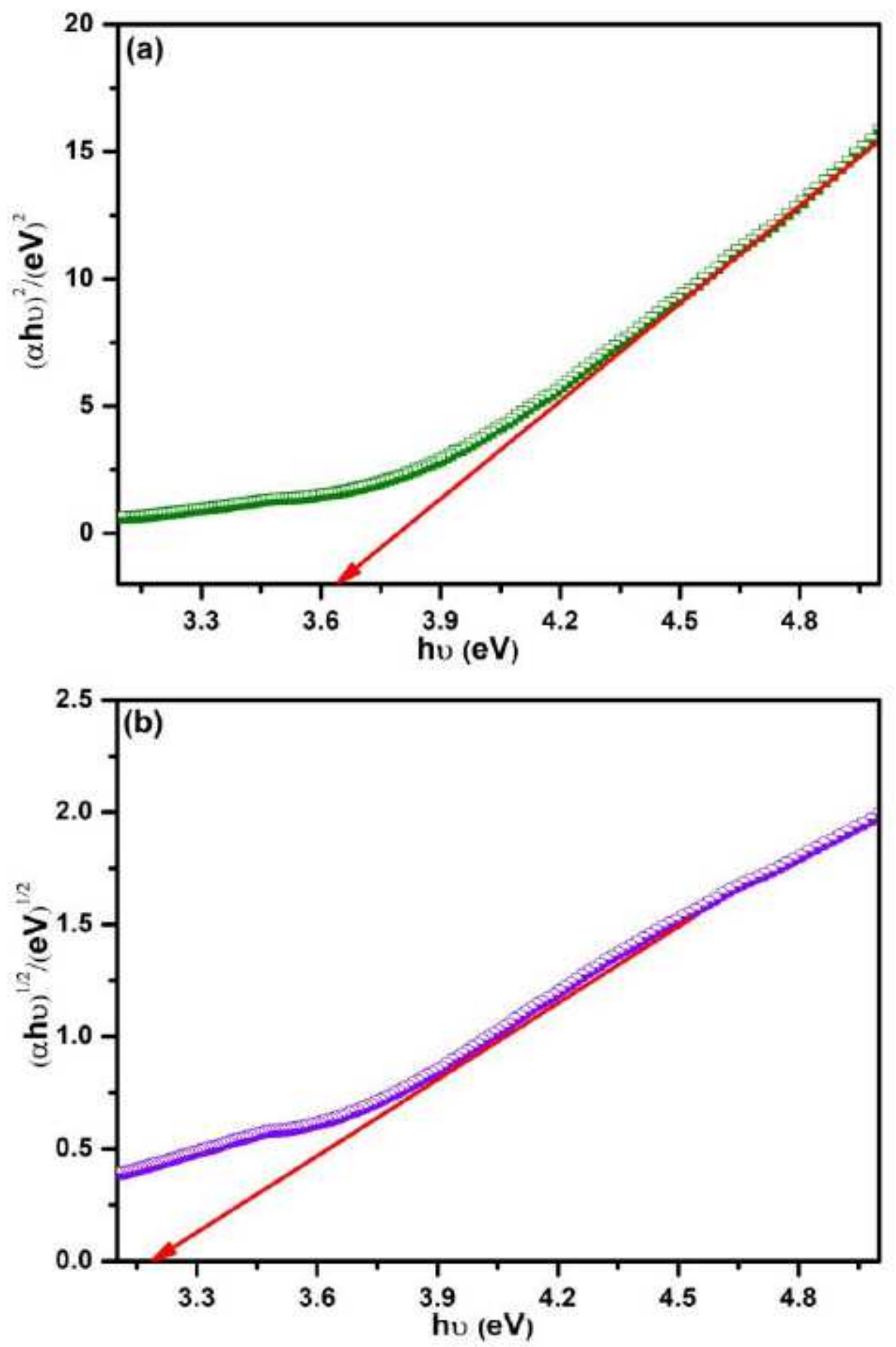

Figure 7

The relationship of $(a h \rrbracket) n$ versus photon energy $h \rrbracket$ to determine the optical band gap of SBSO:3\%Tm3+ for (a) direct and (b) indirect allowed transitions 

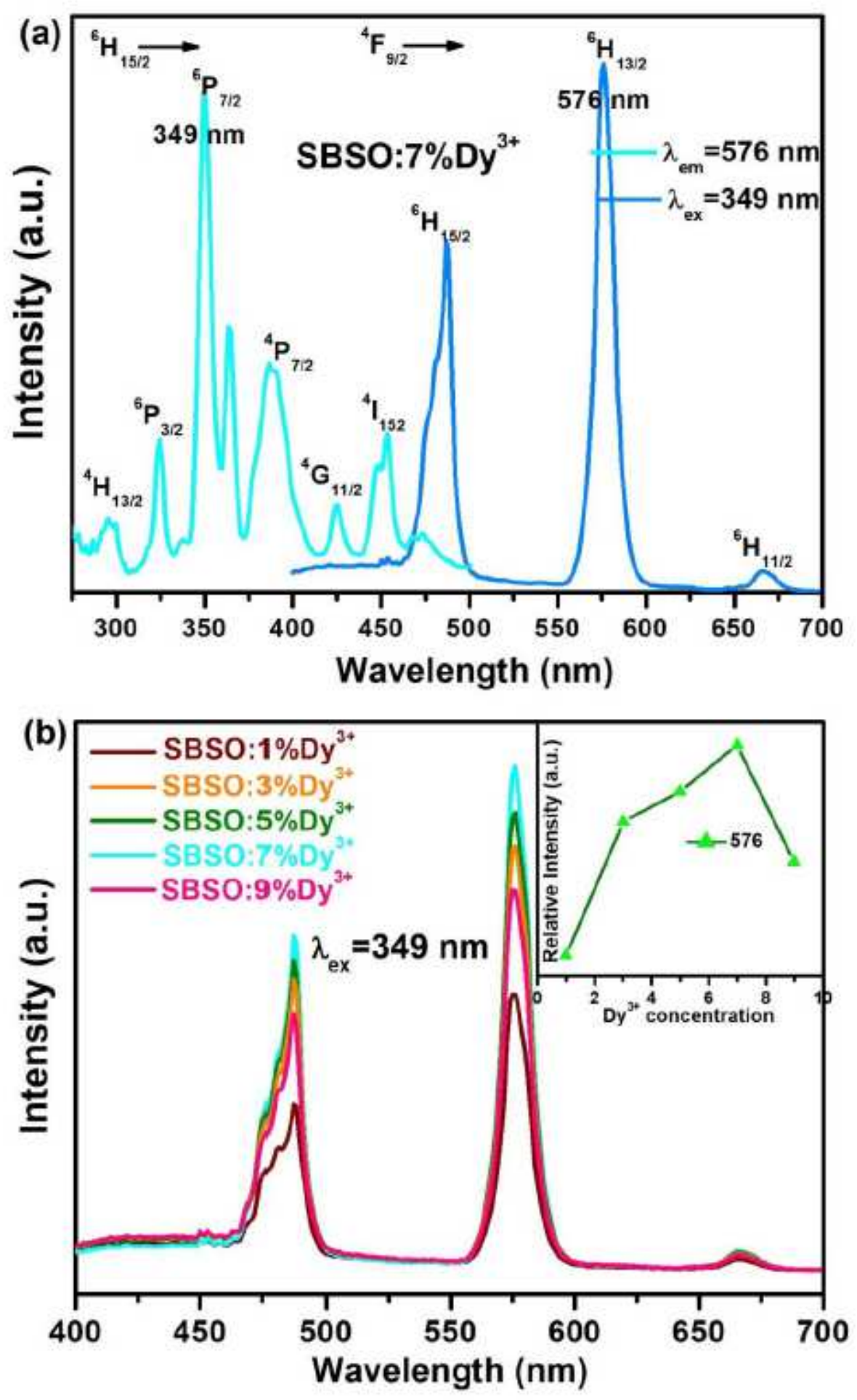

Figure 8

(a) PL and PLE spectra for SBSO:7\%Dy3+ (b) PL spectra for SBSO:x\%Dy3+ ( $x=1-9)$ with different Dy3+ doped concentrations, the inset shows the optimal concentration curve of Dy3+ 

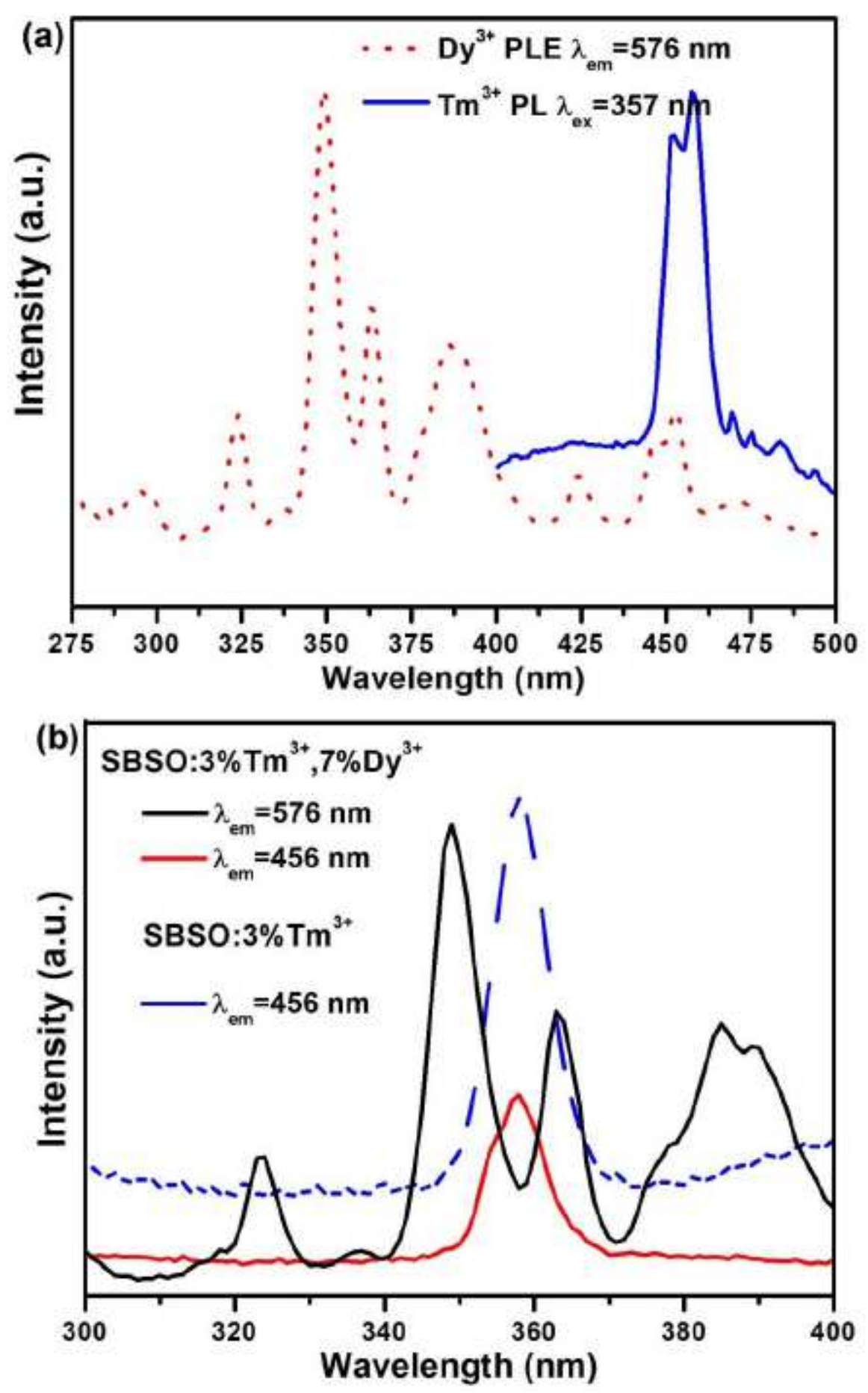

Figure 9

(a) The corresponding spectral overlapping of Tm3+ PL spectrum for SBSO:Tm3+ and the Dy3+ PLE spectrum for SBSO:Dy3+ (b) The PLE spectra of SBSO:Tm3+,Dy3+ ( $\lambda e m=456$ and $576 \mathrm{~nm})$ and SBSO:Tm3+ $(\lambda e m=456 \mathrm{~nm})$ 

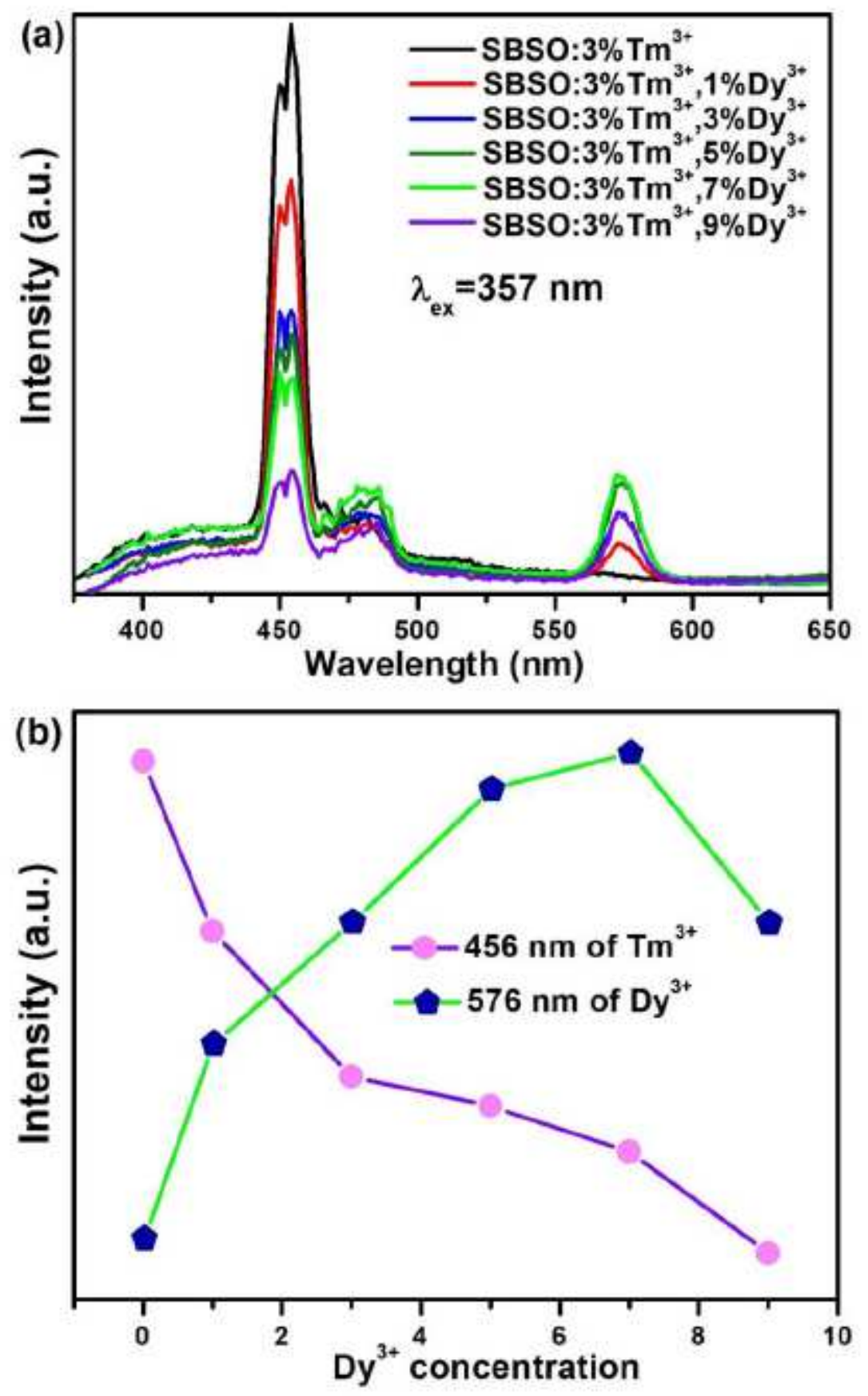

Figure 10

(a) The emission spectra of SBSO:3\%Tm3+,y\%Dy3+ ( $y=0-9)$ with different Dy3+ doped concentrations (b) Dependence of emission intensity on Dy3+ concentrations 


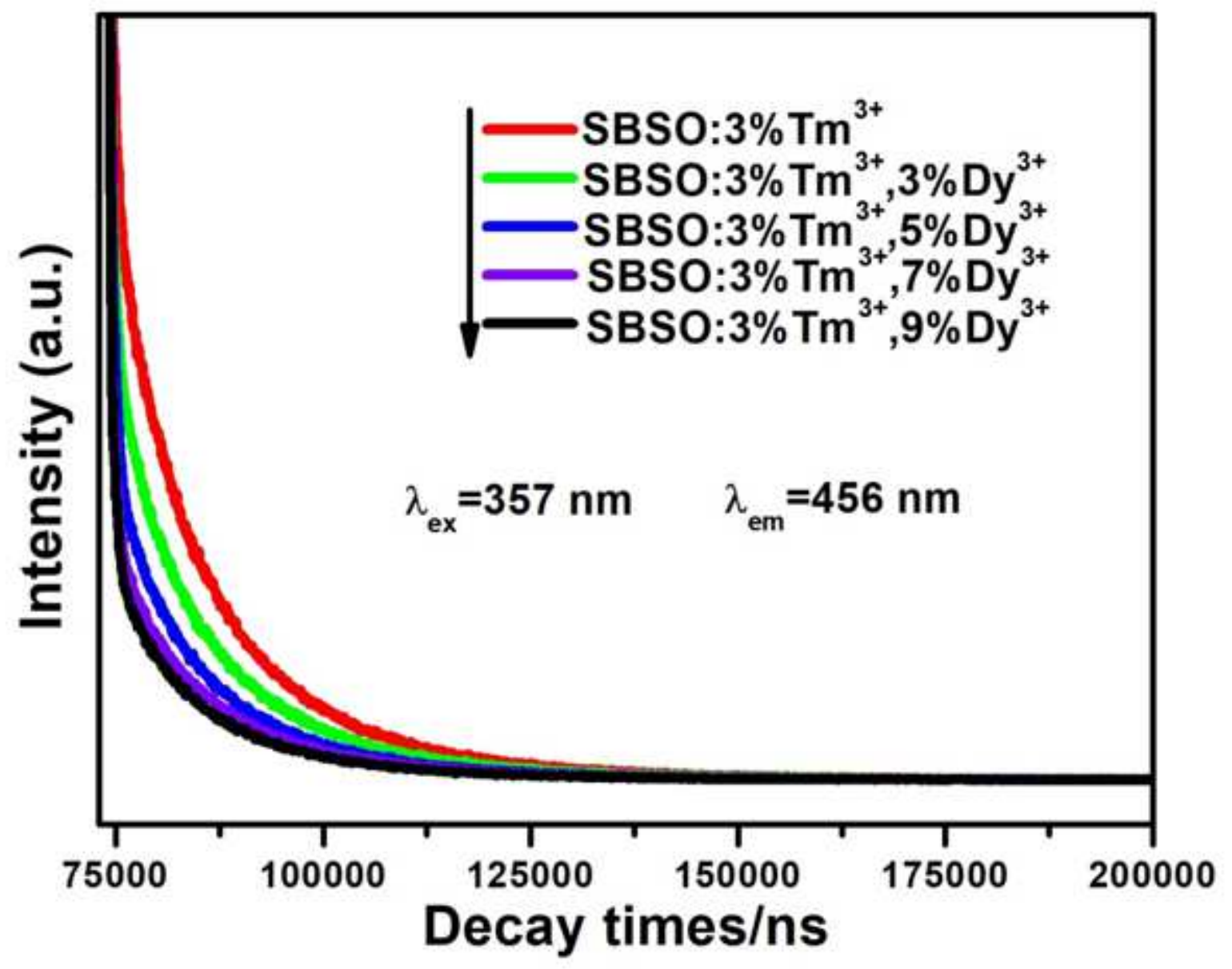

Figure 11

The decay curves for the luminescence of Tm3+ in SBSO:3\%Tm3+, y\%Dy3+ excited at $357 \mathrm{~nm}$, monitored at $456 \mathrm{~nm}$ 


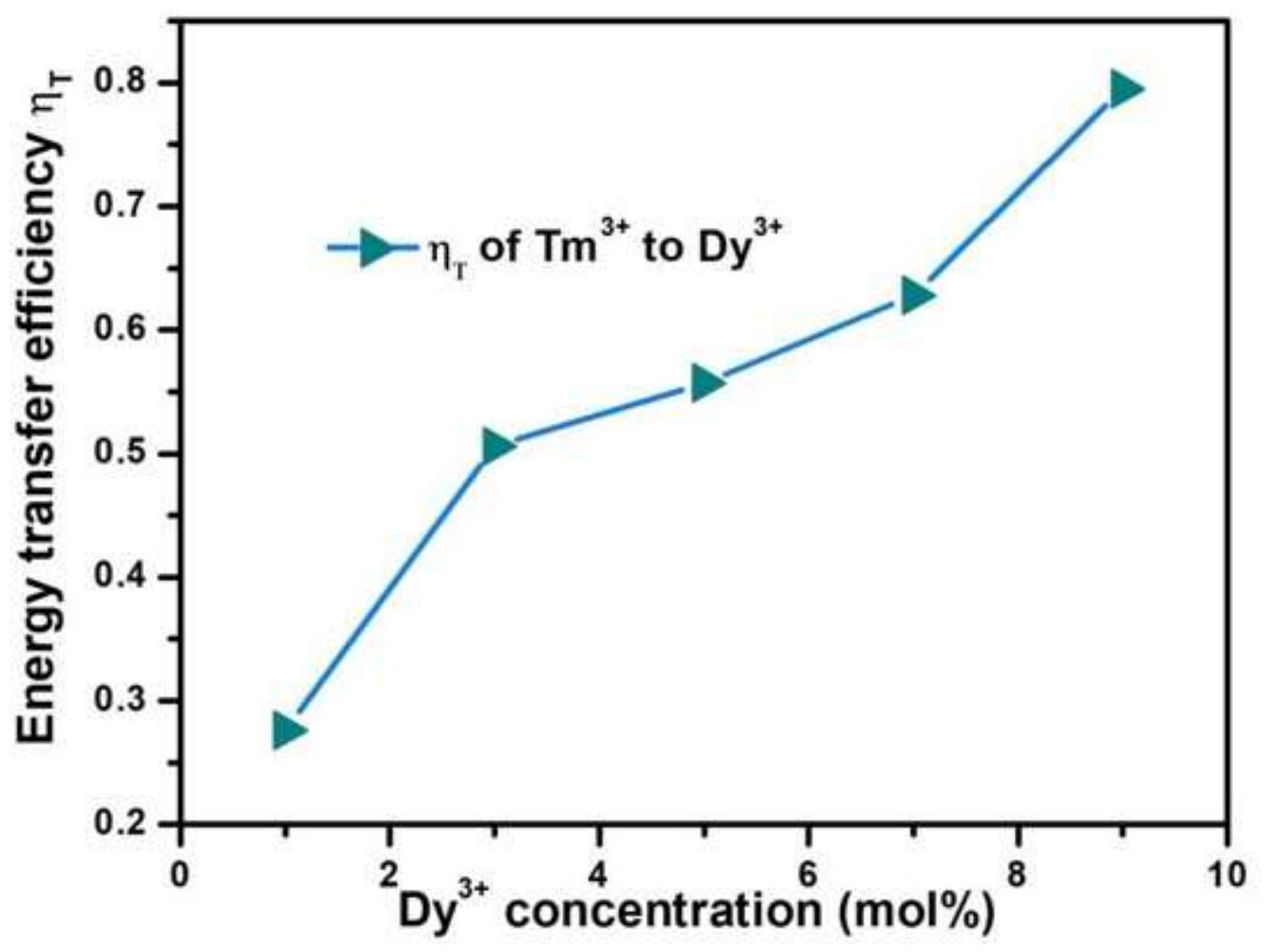

Figure 12

Dependence of energy transfer efficiency $\eta T$ on Dy3+ concentrations in SBS0:3\%Tm3+, y\%Dy3+ 


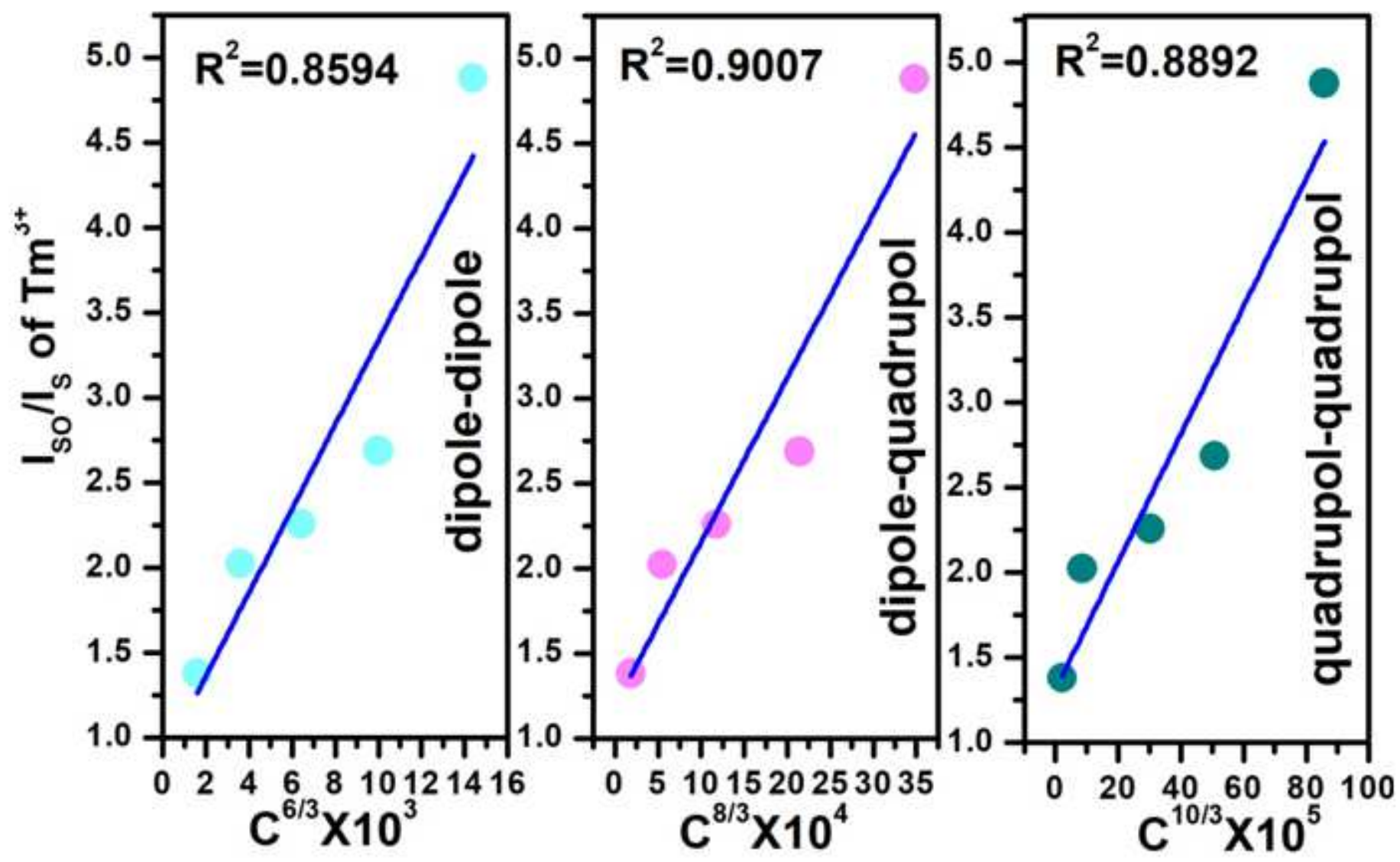

Figure 13

Dependence of Iso/Is of Tm3+ on (a) C6/3, (b) C8/3, and (c) C10/3 

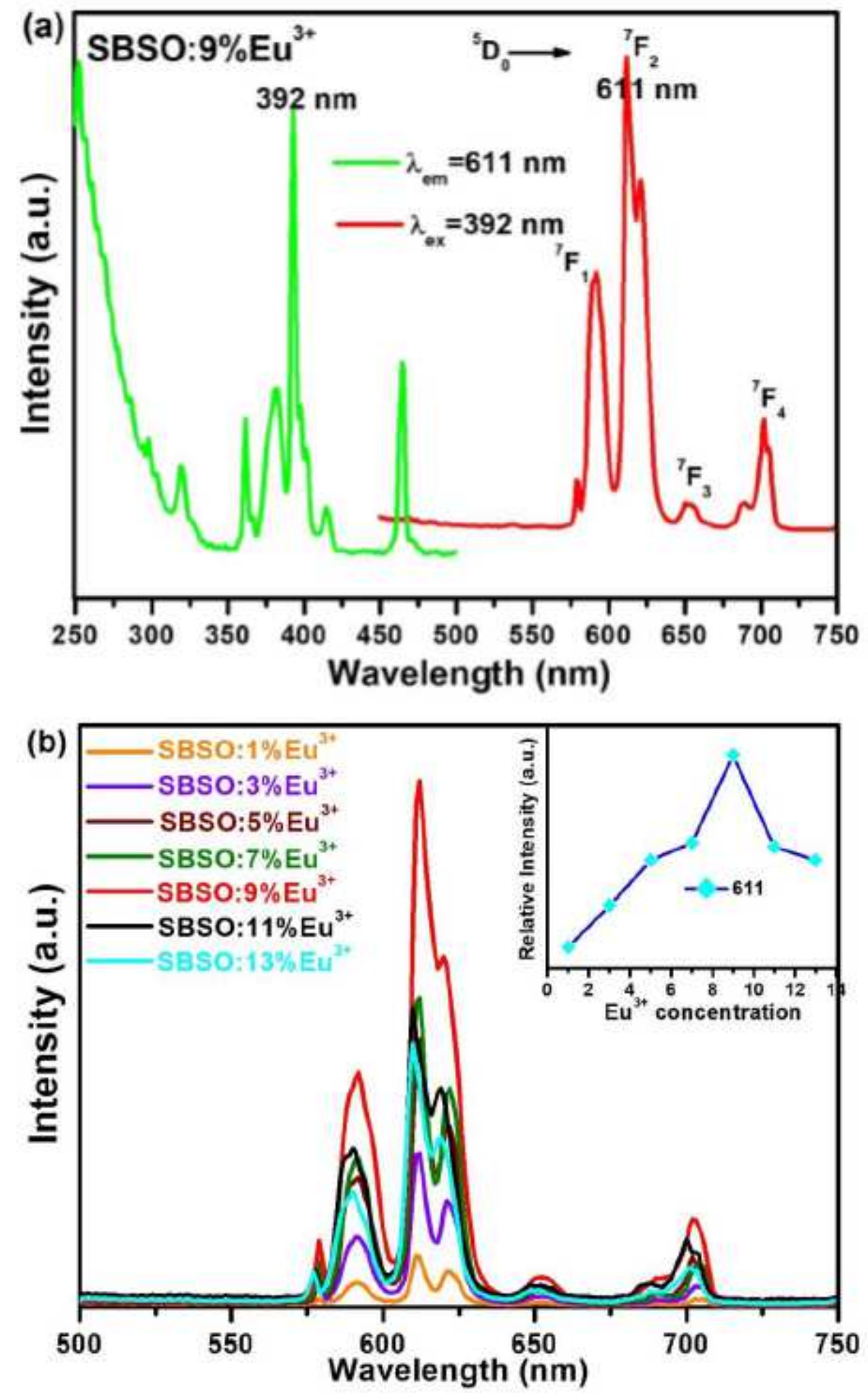

Figure 14

(a) The excitation and emission spectra of SBSO:9\%Eu3+ (b) The emission spectra of SBSO:x\%Eu3+ $(x=1-13)$, the inset shows the relative intensity of Eu3+ ion with different Eu3+ concentrations 


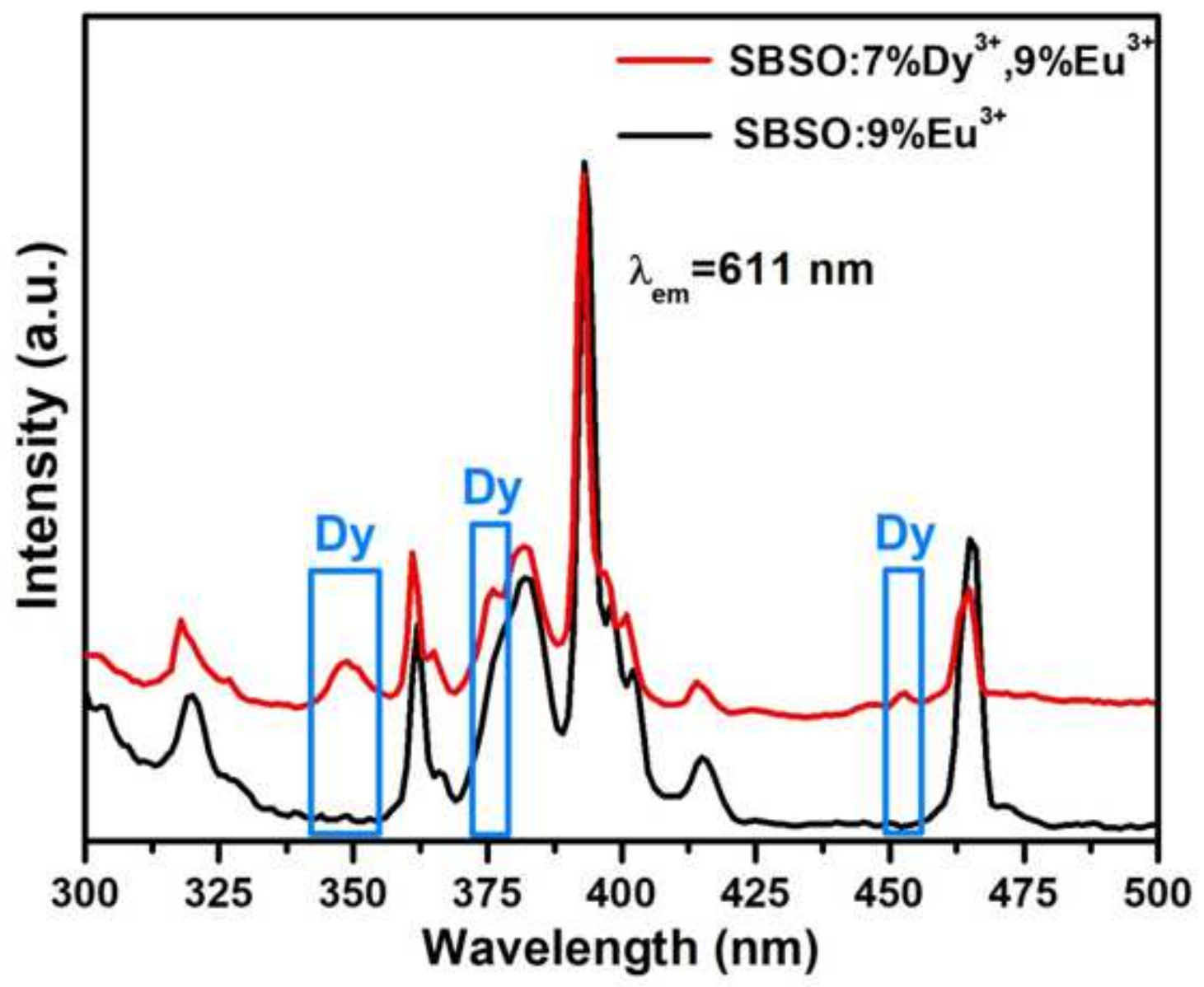

Figure 15

The PLE spectra for SBSO:9\%Eu3+ and SBSO:7\%Dy3+, 9\%Eu3+ both monitored by the emission of Eu3+ at $611 \mathrm{~nm}$ 

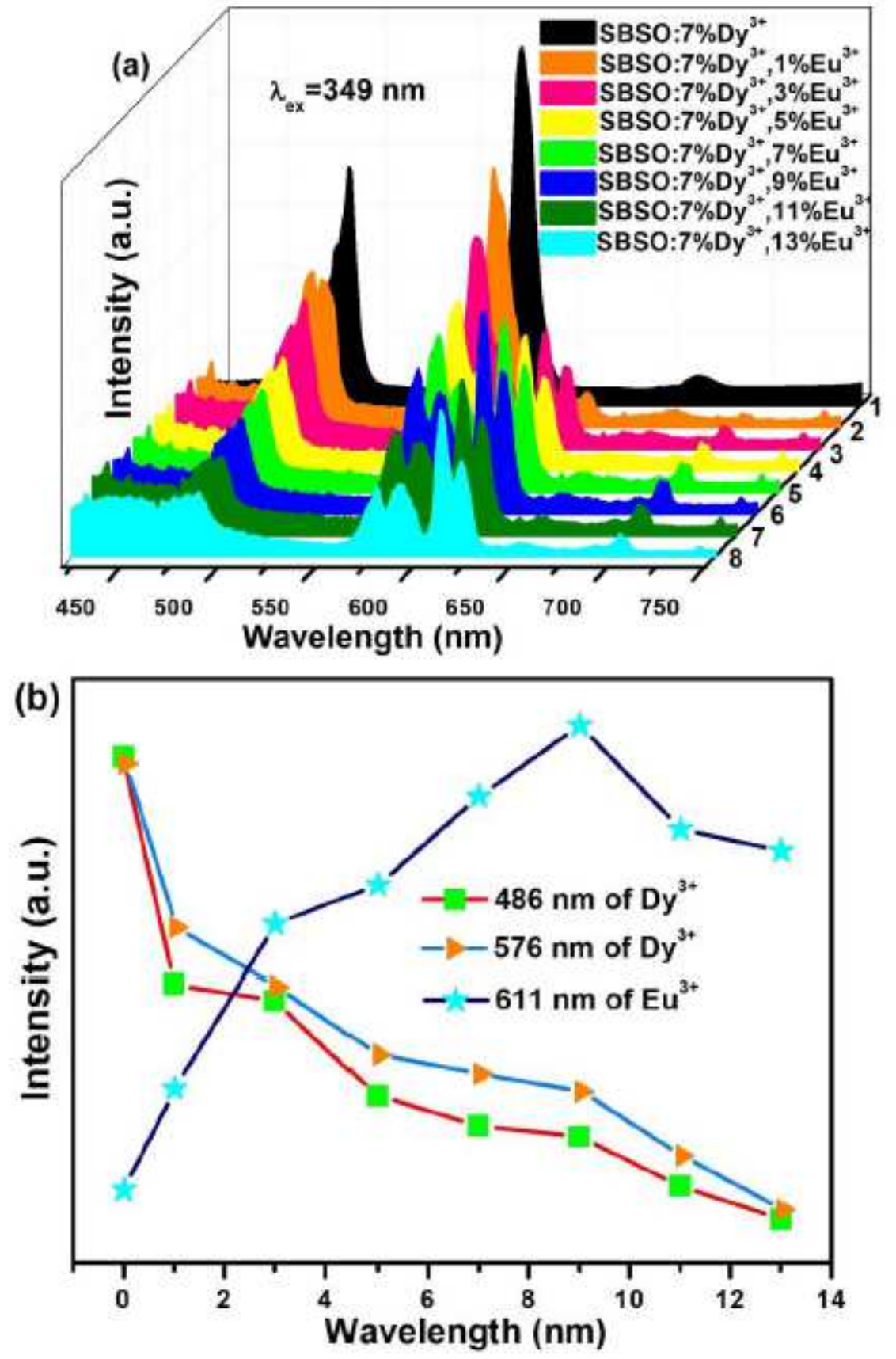

Figure 16

(a) PL spectra for SBS0:7\%Dy3+, y\%Eu3+ phosphors with different Eu3+ doped concentrations (b) Dependence of emission intensity on Eu3+ concentrations 


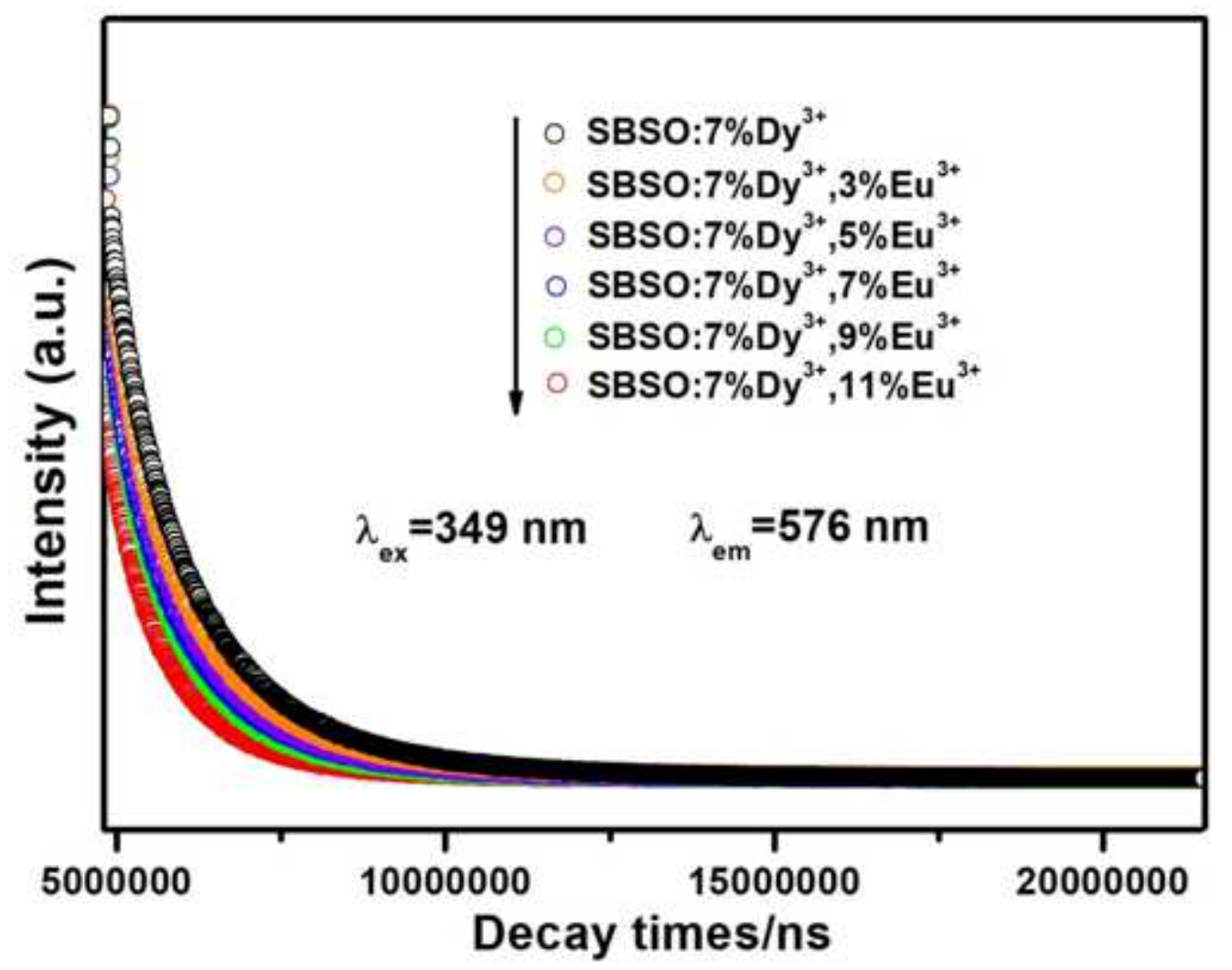

Figure 17

Decay lifetime tests of SBS0:7\%Dy3+, yEu3+ detected at $576 \mathrm{~nm}$ for Eu3+ emission excited at $349 \mathrm{~nm}$ 


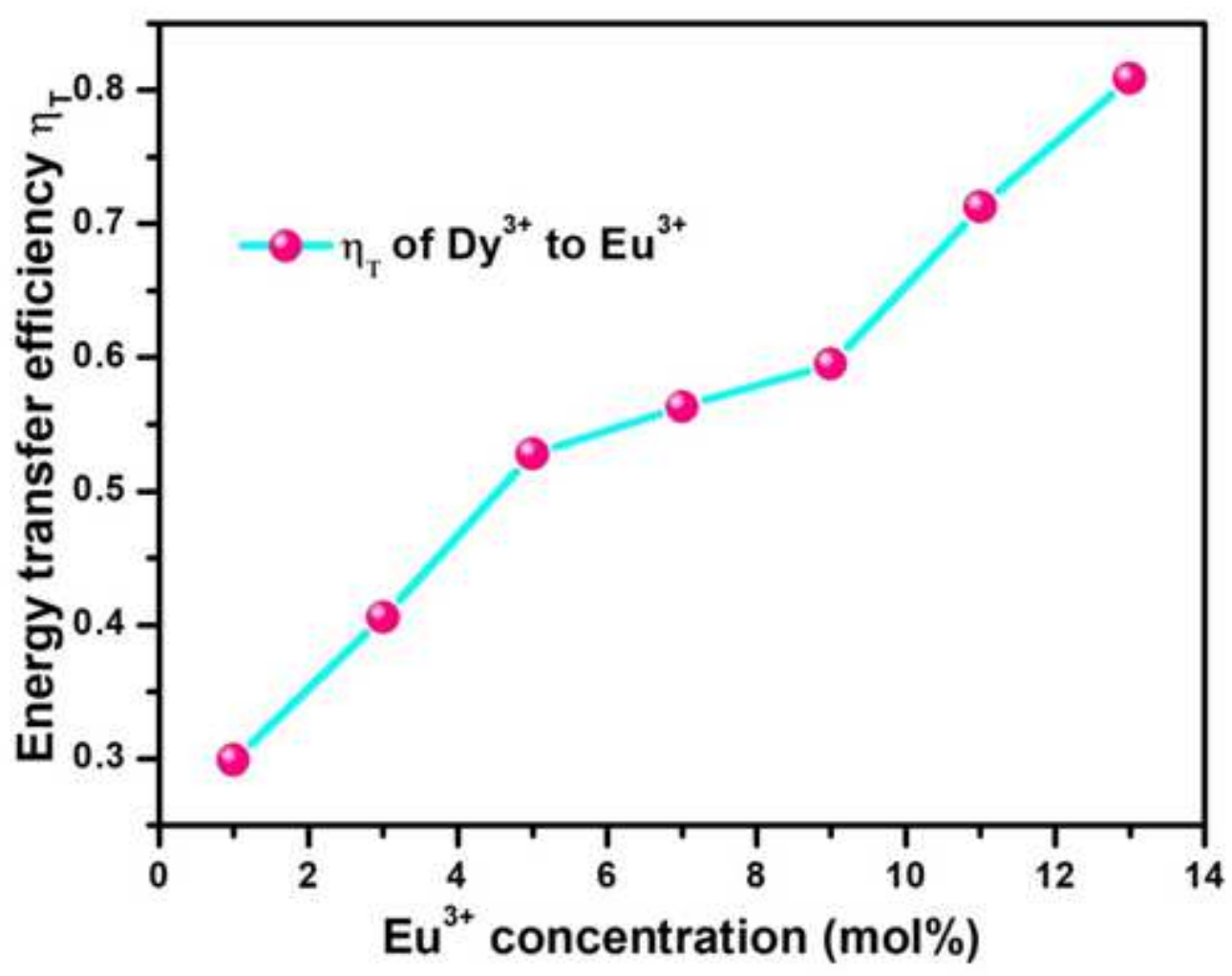

Figure 18

Energy-transfer efficiency of Dy3+-Eu3+ on Eu3+ doping concentration for SBS0:7\%Dy3+, y\%Eu3+ phosphors 

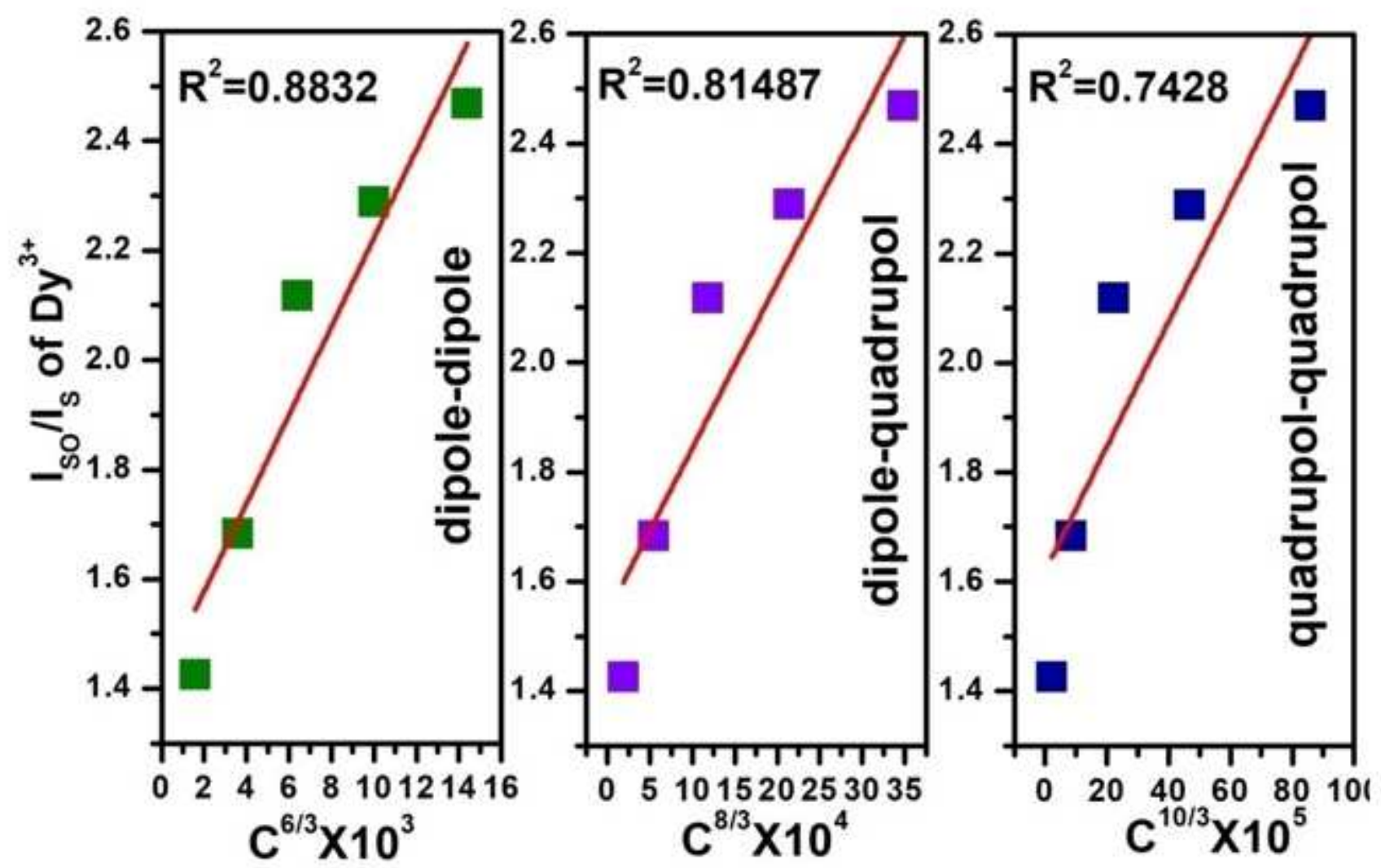

Figure 19

Dependence of Iso/Is of Dy3+ on (a) C6/3, (b) C8/3, and (c) C10/3 


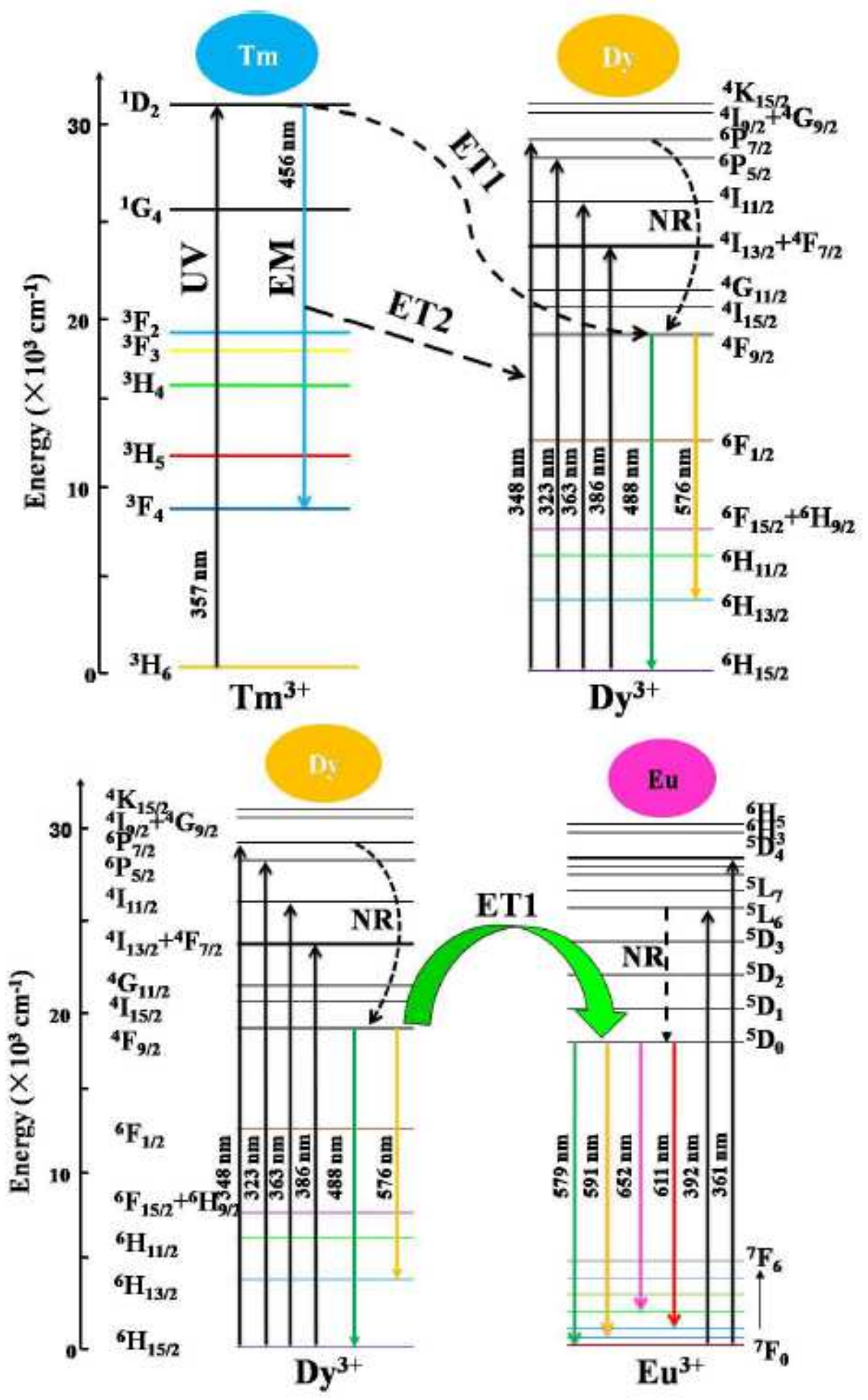

Figure 20

Schematic energy-level diagram of energy transfer mechanism in the SBSO:Tm3+/Dy+/Eu3+ system 


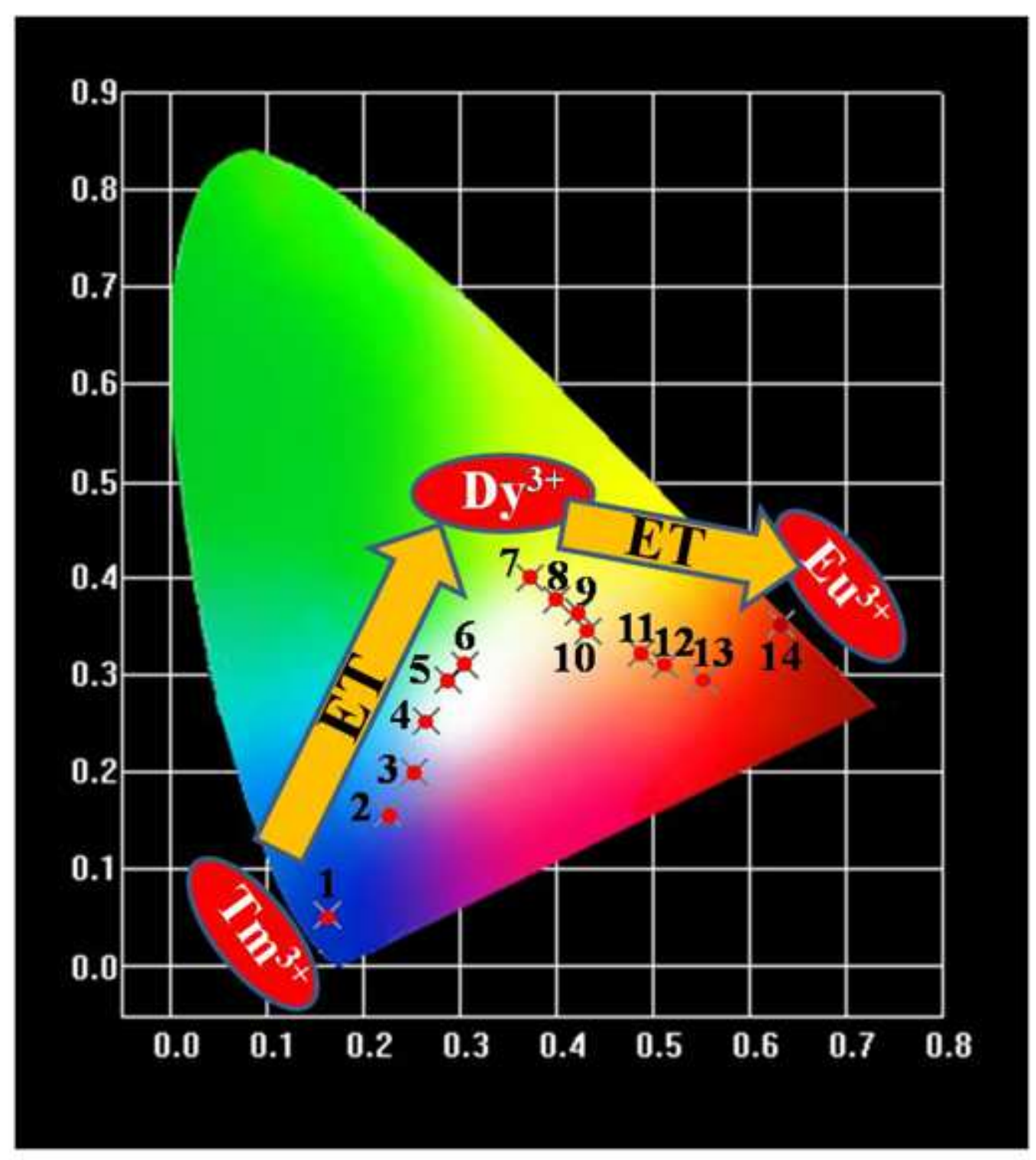

Figure 21

The CIE chromaticity coordinates for SBSO:Tm3+/Dy3+/Eu3+ phosphors under 357 and $349 \mathrm{~nm}$ excitation 

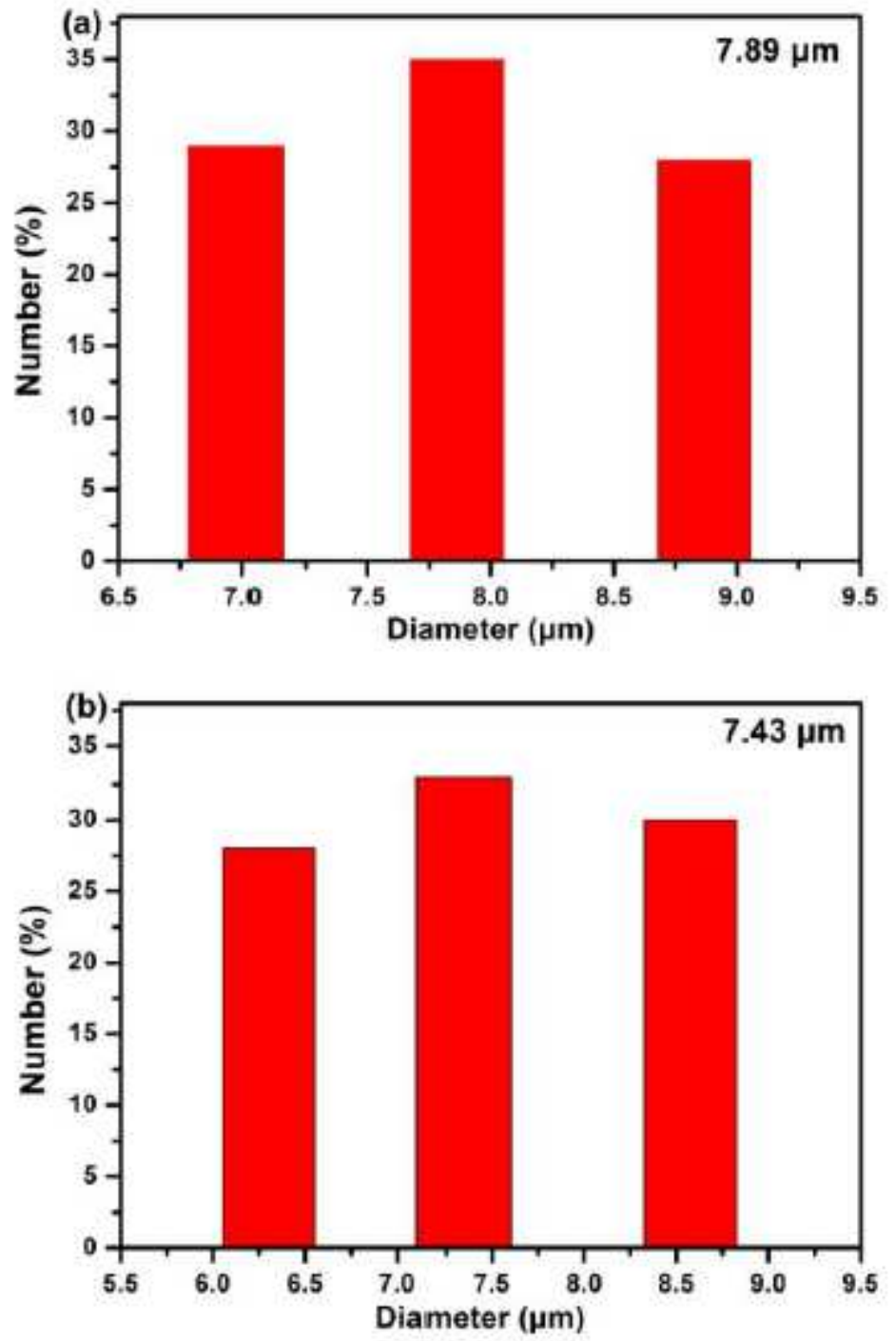

Figure 22

The particle size distribution of (a) SBS0:3\%Tm3+, 7\%Dy3+ and (b) SBS0:7\%Dy3+, 9\%Eu3+ phosphors, the average diameters are 7.89 and $7.43 \mu \mathrm{m}$, respectively 